\title{
Advanced DSP Control of Induction Motors using Kalman Filter
}

by

Shiping Zhu

\author{
A project \\ presented to Ryerson University \\ in partial fulfillment of the \\ requirement for the degree of \\ Master of Engineering \\ in the Program of \\ Electrical and Computer Engineering \\ Toronto, Ontario, Canada, 2003
}

CShiping Zhu, 2003 
UMI Number: EC53454

\section{INFORMATION TO USERS}

The quality of this reproduction is dependent upon the quality of the copy submitted. Broken or indistinct print, colored or poor quality illustrations and photographs, print bleed-through, substandard margins, and improper alignment can adversely affect reproduction.

In the unlikely event that the author did not send a complete manuscript and there are missing pages, these will be noted. Also, if unauthorized copyright material had to be removed, a note will indicate the deletion.

\section{UMI}

UMI Microform EC53454

Copyright 2009 by ProQuest LLC

All rights reserved. This microform edition is protected against unauthorized copying under Title 17, United States Code.

ProQuest LLC

789 East Eisenhower Parkway

P.O. Box 1346

Ann Arbor, Ml 48106-1346 


\section{Author's Declaration}

I hereby declare that I am the sole author of this research paper.

I authorize Ryerson University to lend this research paper to other institutions or individuals for the purpose of scholarly research.

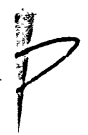

I further authorize Ryerson University to reproduce this research paper by photocopying or by other means, in total or in part, at the request of other institutions or individuals for the purpose of scholarly research.

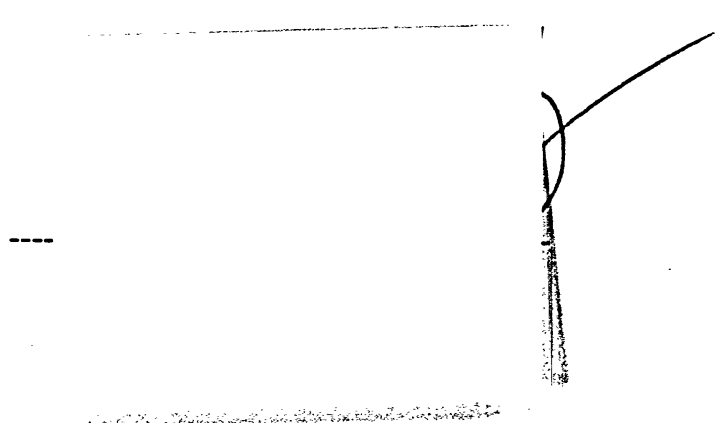




\section{Instructions on Borrowers}

Ryerson University requires the signatures of all persons using or photocopying this research paper. Please sign below, and give address and date. 


\title{
Advanced DSP Control of Induction Motors using Kalman Filter
}

\begin{abstract}
This research paper presents a novel method for the speed control of induction motors without using a speed sensor. The rotor speed can be accurately computed using an optimal control observer named the Kalman filter designed in this research paper. This replaces a speed sensor and eliminates the difficulty of the sensor installation in many applications. This research paper presents an advanced field oriented control of induction motors, based on a specific $d-q$ coordinate model with the d-coordinate chosen to be in line with the rotor flux and the q-coordinate chosen to be $90^{\circ}$ lagging. The position of the rotor flux can be accurately computed using the Kalman filter. This eliminates the position sensors required to monitor the flux. This research paper shows that as a result of this specific d-q transformation, the motor torque is proportional to the product of the rotor flux and the q-coordinate stator current. This significantly simplifies the induction motor control, such that the rotor flux is simply controlled by regulating the flux-related d-coordinate stator current and the motor torque is controlled just by regulating the q-coordinate stator current. This research paper presents a computational efficient recursive algorithm for Kalman filter which is specifically designed for the induction motor control. The Kalman filter provides the minimum variance state estimation and tolerates induction motor modeling and measurement errors. The Kalman filter can process all available measurements regardless of their precision (only two input current measurements required for the motor control), and provides a quick and optimal estimate of the variables of interest (the rotor speed and flux selected as outputs), as well as achieves a fast convergence. This research paper presents the digital signal processor (DSP) implementation of the field oriented control of induction motors using Kalman filter. The hardware requirements and all software modules are detailed. The experimental verification of the control method designed in this research paper is provided. Typical measurements are given to demonstrate the efficiency of the novel control presented in this research paper.
\end{abstract}

Key words: field oriented control, Kalman filter, DSP control, PID regulator 


\section{Acknowledgments}

I would like to express my sincere gratitude and appreciation to my supervisor Professor Richard Cheung for his guidance in my study and project. I cordially thank Professor Bin Wu for his advice and help in my study period.

At the same time I would like to thank my classmates or friends in the laboratory of power electronics at Ryerson University for their help or assistance in my project.

Specially thanks to my wife and son for their full support. 


\section{Contents}

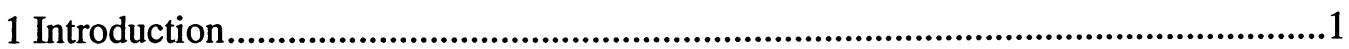

1.1 DSP Control of Induction Motors …........................................................2

1.2 Speed Sensorless Control of Induction Motors..............................................3

1.3 Novel Control Method for Induction Motors..................................................4

1.4 Outline of the Research Paper...................................................................6

2 Advanced Field Oriented Control of Induction Motors .......................................8

2.1 3-phase to 2-phase Transformation ...............................................................9

2.2 Space Vector PWM Control of Motor Drive Inverter .................................11

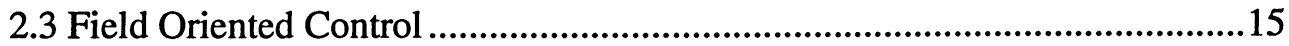

2.4 Adaptive PID Regulator......................................................................17

3 Kalman Filter for Speed Sensorless Control of Induction Motors.........................20

3.1 Basics of Kalman Filter .............................................................................20

3.2 Induction Motor Model for Implementation of Kalman Filter ......................23

4 Digital Signal Processor Implementation of Speed Sensorless Field Oriented

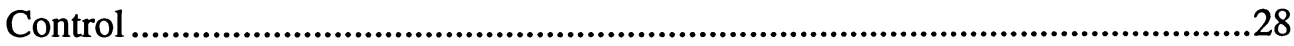

4.1 Overview of DSP FOC Operations .......................................................28

4.2 Hardware Implementation of Field Oriented Control ................................29

4.3 DSP Software Implementation of Field Oriented Control.............................31

4.4 Debugging of Software and Hardware .....................................................33

5 Experimental Verification of Field Oriented Motor Control using Kalman Filter ...36

5.1 Internal States of Closed-Loop Control ....................................................36

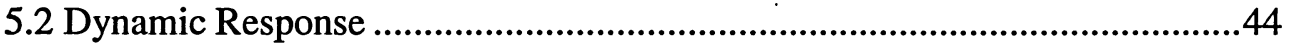

5.3 Speed Control Accuracy ...................................................................46

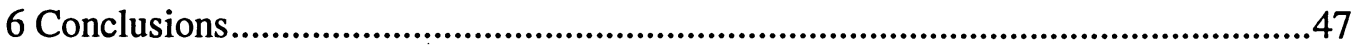

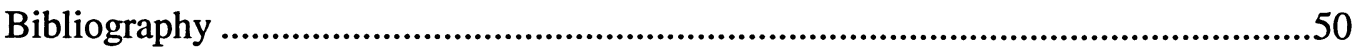

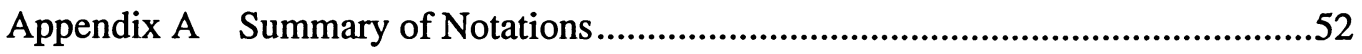

Appendix B Induction Motor Parameters..........................................................53

Appendix C Characteristics of PID Control ......................................................54

Appendix D Assembly Code for TMS320LF2407 ............................................55 


\section{List of Figures}

Figure 1: Methods of Sensorless Speed Control.............................................................4

Figure 2: The Control Structure of FOC with Kalman Filter ...........................................5

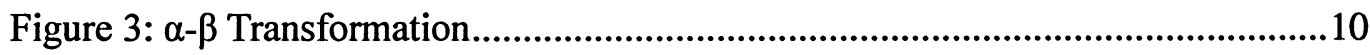

Figure 4: Park Transformation .......................................................................11

Figure 5: Three-phase Voltage Source Inverter ..........................................................12

Figure 6: Configuration of Space Vector PWM.......................................................13

Figure 7: Example of Space Vector PWM pattern .....................................................15

Figure 8: Field Oriented Control of Induction Motors...............................................16

Figure 9: Relationship between Current, Voltage and Rotor Flux................................16

Figure 10: Sensorless Controller for Induction Motors ................................................17

Figure 11: The Adaptive PID Control ...................................................................18

Figure 12: Principle of Kalman Filter ................................................................21

Figure 13: Extended Kalman Filter Algorithm..........................................................23

Figure 14. Experiment Setup for Sensorless Control System......................................29

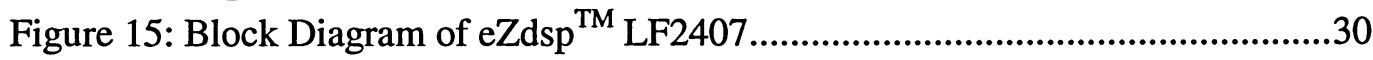

Figure 16: Software Flowchart ..................................................................................31

Figure 17: EKF Software Structure for an Induction Motor ........................................32

Figure 18: Overview of Phase 2 Software Flow......................................................34

Figure 19: Overview of Phase 2 Workspace.............................................................35

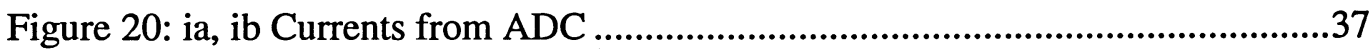

Figure 21: Currents from Clarke Transformation ......................................................37

Figure 22: Currents Estimated by Kalman Filter ........................................................38

Figure 23: Flux Circle Estimated by Kalman filter....................................................38

Figure 24: Currents from Park Transformation ...........................................................39

Figure 25: Speed Control.................................................................................39

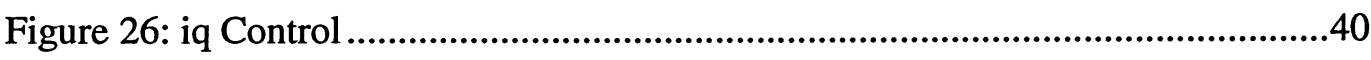

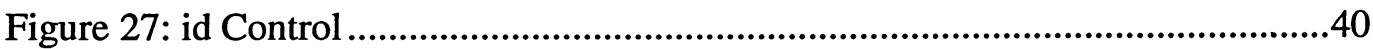

Figure 28: Voltage Outputs from id, iq Regulators................................................

Figure 29: Ualfa, Ubeta from Inverse Park Transformation..........................................41

Figure 30: Duty Ratios of PWM1, PWM3 and PWM5 in Carrier Period....................42

Figure 31: PWM Outputs of IGBT Power Module.....................................................43

Figure 32: Line to Line Voltages of the Induction Motor ...........................................43

Figure 33: Speed Step Response ...............................................................................44

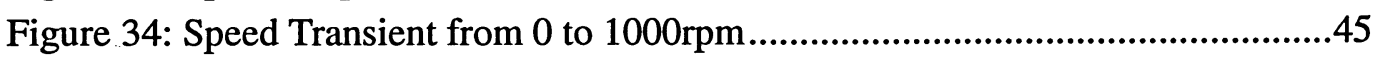

Figure 35: Speed Reversion..................................................................................45

Figure 36: The Model of an Induction Motor...........................................................53 


\section{List of Tables}

Table 1: Steady State Performance of Speed Sensorless Control. . . . . . . . . 46

Table 2: Summary of Notations Used in this Research Paper . . . . . . . . . . 52 


\section{Chapter 1}

\section{Introduction}

Induction motors are popular in the industry because they are reliable, rugged, low cost, and virtually maintenance free. Market analysis shows that most of industrial motor applications use induction motors. However, the use of induction motors may have several difficulties, which include control disadvantages due to complex motor model and nonlinear motor parameters caused by saturation and variations with temperature. In order to overcome these difficulties, many methods have been proposed for the control of induction motors [1-5]. The field-oriented control (FOC) is the most popular one. This control requires the instantaneous measurements of the motor speed, voltages and currents. The speed of the motor is usually measured using a speed/position sensor. The installation of the sensor not only increases the motor drive cost and the sensor maintenance cost, but also the installation could be very difficult or impractical for some applications such as the pumps used in oilrigs.

This research paper presents a novel method for controlling the speed of induction motors without the need of a speed or position sensor. The information of the motor speed is obtained by processing the stator voltages and currents which are simply measured at the motor terminals or at the supply terminals. Induction motor drives without speed or position sensors will increase the drives reliability and reduce their costs. This research paper has developed an efficient control for induction motors using a closed-loop optimal control observer named the Kalman filter to compute the motor speed and position, as well as using an adaptive feedback regulator to provide a reliable and accurate speed control.

The following lists the key work carried out in this research paper.

a. Full investigation of Kalman filter for the control of induction motors.

This research paper provides a full investigation of the practical application of Kalman filter for the control of induction motors. There has been very limited information about the use of Kalman filter for induction motor drives, possibly because of the difficulty of understanding the theory of the Kalman filter. This research paper research provides a detailed study of the control theory of the Kalman filter and its suitability for the control of induction motors. This research provides a concrete finding that Kalman filter is well suitable for induction motor control.

b. Detailed formulations of the specific field oriented control with Kalman filter for real-time control of induction motors. 
This research paper develops all formulations required for the specific field oriented control of induction motors using Kalman filter. In particular, a recursive algorithm of the Kalman filter is given in the research paper. The algorithm and its corresponding formulations has provided an accurate computation of the rotor speed, the flux linkage, and the flux angle which are the essential components for the specific field oriented control of induction motors.

c. DSP implementation of the specific field oriented induction motor control.

This research paper develops the software required for the implementation of the specific field oriented motor control in DSP TMS320LF2407. The software is developed in modules which can be easily modified for different induction motor drive applications.

d. Real-time verification of the specific field oriented control using.Kalman field.

A prototype of the induction motor drive is built to verify the specific field oriented control using Kalman filter. Typical measurements are given to demonstrate the efficiency of the novel control designed in this research paper.

This chapter provides an introduction of the control method developed in this research paper. The following outlines the sections in this chapter.

Section 1.1 discusses the digital signal processor (DSP) control of induction motors.

Section 1.2 assesses the research in speed sensorless control of induction motors.

Section 1.3 introduces the novel control method designed in this research paper. This control combines the field oriented control and the Kalman filter.

Section 1.4 shows the structure of this research paper.

\subsection{DSP Control of Induction Motors}

Induction motor controls were traditionally designed and implemented with analog components. There are several drawbacks with analog controllers including component aging and drifts. Analog controllers are usually hard-wired circuits that make modifications or upgrades fairly difficult. Also the implementation of advanced control algorithms using analog circuits requires an excessive number of components. On the other hand, digital control offers improvements over the analog control. The effect on the control due to analog component characteristic variations can be eliminated in the digital control. Upgrades in the digital control can fairly easily conducted in software, and the number of control circuit components required is significantly reduced since the digital controller can handle many control functions in a single chip.

Digital signal processor (DSP) can provide high speed and high accuracy control signals. Fixed-point DSPs are preferable in the cost-effective induction motor control, because 
fixed-point DSPs cost much less than the floating-point ones and for most applications, the dynamic range of 16 bits is sufficient. If needed for certain small portions in the control computation, the dynamic range can be increased in the fixed-point processor by carrying out floating-point calculations.

The performance of an induction motor strongly depends on the characteristics of its control. DSPs can be used to enhance the real-time control of induction motors without the use of any electromechanical speed sensors. The DSP controller can reduce the number of control circuit components and can optimize the drive performance. The DSP for motor drive applications can perform the following.

1) Handle complex control schemes such as implementation of sensorless motor control algorithms to eliminate the need of a speed or position sensor.

2) Implement communication functions for fault and status information.

3) Re-program control schemes for different applications.

4) Generate high-resolution PWM signals for efficient control of the power electronic inverter and reduction of harmonics.

5) Provide a single chip drive control system and reduce the drive system cost.

\subsection{Speed Sensorless Control of Induction Motors}

The world market of induction motor drives is over ten thousand million US dollars annually with a rapid growth. Current researches on motor drives have focused on the elimination of the speed sensor at the motor shaft without deteriorating the dynamic performance of the drives $[6,7]$. The speed estimation is of particular interest with the induction motor drives where the mechanical speed of the rotor is generally different from the speed of the revolving magnetic field. The advantages of speed-sensorless induction motor drives include reduction of hardware complexity and cost, increase of control-circuit noise immunity and drive reliability, and reduction of maintenance requirements. Operations in the hostile environments mostly require motor drives without speed/position sensors.

Figure 1 shows the block diagram of the speed-sensorless induction motor control. Basically there are two commonly-used control methods: the voltage-to-frequency (V/f) control and the field oriented control (FOC). Both methods for the speed sensorless control require a speed estimation algorithm. In the V/f control, the ratio of the stator voltage to stator frequency is kept constant using a feed forward control to maintain the magnetic flux in the motor at a desired level. This control is simple but it only can satisfy moderate motor dynamic requirements. On the other hand, high motor dynamic performance can be achieved using the FOC control which is also called the vector control. The stator currents are injected at a well-defined phase angle with respect to the spatial orientation of the rotating magnetic field, thus overcoming the complex dynamic properties of the induction motor. The spatial location of the magnetic field, that is the field angle, is difficult to measure. 
There are several models and algorithms that can be used for the estimation of the field angle, for example the open-loop estimator such as model reference adaptive system (MRAS), or the close-loop observer such as the Kalman filter. The induction motor control using the field orientation usually refers to the rotor field. This research paper presents a novel rotor field oriented control.

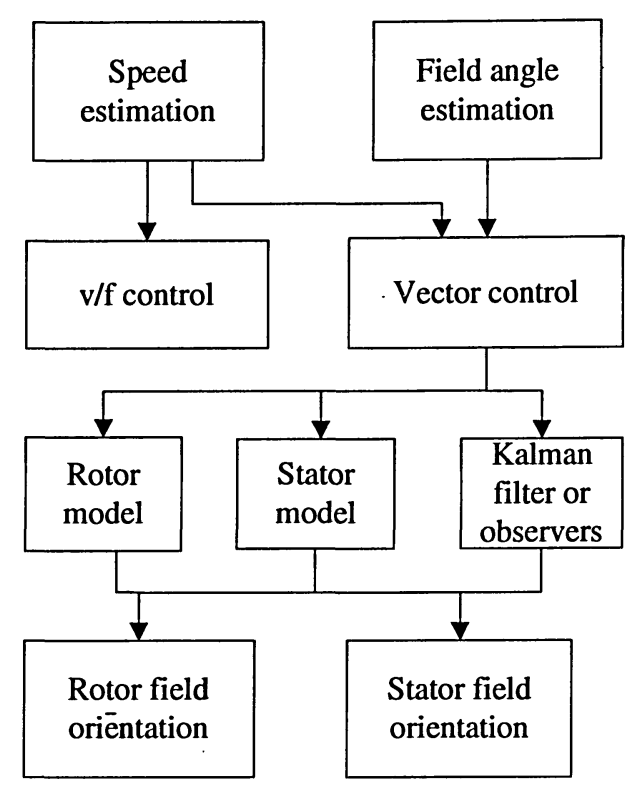

Figure 1: Methods of Sensorless Speed Control

\subsection{Novel Control Method for Induction Motors}

This research paper investigates the real-time field oriented control (FOC) of induction motors using a Kalman filter and an adaptive feedback controller. This FOC control has been successfully implemented in the Power Electronic Laboratory at Ryerson University. Figure 2 shows the basic control structure. The main contribution of this research paper consists of achieving accurate sensorless control using a Kalman fiter implemented in a DSP processor.

\subsubsection{Field Oriented Control}

An efficient vector control scheme for induction motors has been implemented in this research paper based on the following three major points:

1) Transform the motor's three-phase ever-changing rotor-position dependent model of control and computation disadvantages into a two-coordinate motor-speed model for efficient computation and control.

2) Formulate the current and voltage space vectors for high performance motor speed and torque control. 
3) Generate the pulse-width-modulation (PWM) signals to accurately control the power electronic inverter that drives the induction motor.

Separately-excited dc motor drives are simple in control, because they independently control of the motor's flux and torque through two separate controls of the field current and the armature current. The vector control would like to make the induction motor drive similar to the dc motor drive with two separate controls of the motor flux and the torque. Furthermore, the vector control method can achieve an accurate steady state and transient control that leads to high dynamic performance of the induction motor in a fast response to the changes of its load.

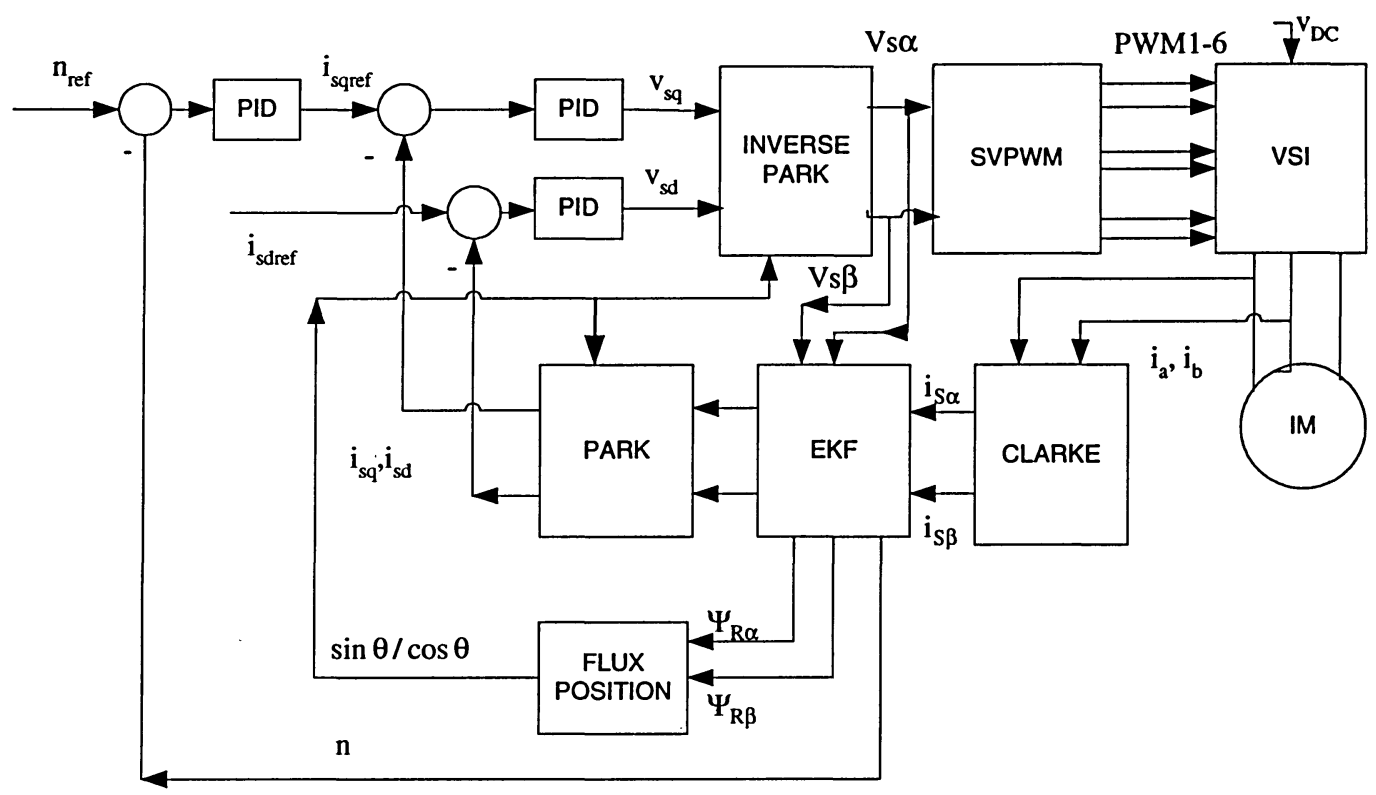

Figure 2: The Control Structure of FOC with Kalman Filter

\subsubsection{Kalman Filter}

The conventional induction motor drive uses electromechanical sensors to measure the position and speed of the rotor. However in many applications such as the pumps used in oil rigs working under the sea, it would not practical to use sensors for the speed or position measurement as it is either technically impossible or extremely expensive. Recently, there have been several proposals addressing this problem and showing that the motor speed can be calculated from the current and voltage inputs to the motor. For a better motor performance, this research paper has designed an observer named the Kalman filter for an efficient control of induction motors. This filter is a statistically optimal observer, which has a good dynamic performance, disturbance rejection, and capability of working in a standstill position.

In general, the implementation of a Kalman filter in the motor control is fairly involved, 
because it requires an accurate model of the induction motor to be calculated in real time. The filter equation to be evaluated involves matrix computations. Digital signal processor (DSP) is especially suitable for this kind of numerical computations. An economical fixed-point DSP TMS320LF2407 from the Texas Instrument is selected for the implementation in this research paper. The design of the Kalman filter of induction motor control is detailed in Chapter3.

\subsubsection{Adaptive PID Controller}

An adaptive controller can accommodate unpredictable changes, whether these changes arise within the system or external to it, such as design and measurement errors or uncertainties. This research paper has designed an adaptive proportional-integral-derivative (PID) control to overcome the control uncertainties and to enhance the quality of the induction motor control. The design of adaptive PID controllers for the induction motor speed and torque control is detailed in Chapter 2.

\subsubsection{Modular Software for Induction Motor Controls}

The benefits of structured modulator software are well known especially for large control systems with many sub-blocks. In examination of various motor control systems, it becomes clear that a large degree of commonality exists between the control software modules. Therefore, if each module is implemented according to well-defined guidelines, then compatibility can be assured across all modules. Since the modularity approach allows efficient software re-uses, efforts have been put on expanding the module library base for greater functionality and features in this research paper. As a form of modularity strategy, the Kalman filter module designed in this research paper can be easily transferred to different induction motor control applications.

\subsection{Outline of the Research paper}

The outline of the following chapters is given below.

Chapter 2 presents an advanced field-oriented control for induction motors. This chapter examines the key components of the field oriented control including the 3-phase to 2-phase transformation, the space vector PWM technique, and the separate induction motor flux and torque control.

Chapter 3 develops the Kalman filter for induction motor control. This chapter details all the formulations required to accurately compute the flux angle for the speed sensorless control of an induction motor. 
Chapter 4 discusses the hardware and software implementation of the novel control scheme designed in this research paper using the DSP TMS320LF2407. This chapter details all key hardware components / circuits and software modules.

Chapter 5 presents the experimental results to demonstrate the accuracy and efficiency of the FOC control designed in this research paper.

Chapter 6 provides a conclusion of this research paper research and recommendations for future work.

Appendix A summarizes the notations used in this research paper.

Appendix B provides the parameter values of the induction motor used in the experiment.

Appendix C shows the characteristics of the PID controllers.

Appendix D gives all software modules for speed sensorless control of the induction motor. 


\section{Chapter 2}

\section{Advanced Field Oriented Control of Induction Motors}

This chapter presents an advanced field-oriented control (FOC) of induction motors, based on mathematical transformations of the standard three-phase motor model into a specific two-phase $d-q$ coordinate model. The three-phase induction motor model is a complex matrix equation with sinusoidal functions of the rotor angle. This model has inherent control and computation disadvantages due to the ever-changing rotor angle. This can be greatly improved with a simple transformation of the three-phase model into a d-q model with the two coordinates rotate with respect to the rotor flux at the synchronous speed. This chapter shows the transformation which, as an extension of the well-known Park's Transformation, provides the induction motor control and computation efficiency.

The three-phase instantaneous stator currents of the induction motor may be modeled as a complex space vector which can be transformed into two currents: the stator d-coordinate current and the stator q-coordinate current, with the d-coordinate chosen to be in line with the rotor flux and the q-coordinate chosen to be $90^{\circ}$ lagging. This chapter shows that as a result of the specific d-q transformation, the motor torque is proportional to the product of the rotor flux and the q-coordinate stator current. The rotor flux of the induction motor can be controlled through the regulation of the flux-related d-coordinate stator current. By maintaining the rotor flux to a desired value, there exists a linear relationship between the motor torque and the q-coordinate stator current. The motor torque can be controlled by regulating the q-coordinate stator current. Therefore, this transformation leads to a simple control structure similar to that of a separately-excited dc motor drive where the flux and the torque of the dc motor can be controlled separately. This transformation also provides significant computational advantage in the field-oriented control of induction motors.

The following outlines the sections in this chapter.

Section 2.1 presents the three-phase to two-phase transformation. This transformation is implemented in two steps. First, transform the three-phase system into the two orthogonal coordinate system. This transformation is known as $\alpha-\beta$ transformation or Clarke's transformation. Second, transform the $\alpha-\beta$ coordinates into the $d-q$ coordinates, with the $d$-coordinate chosen to be in line with the rotor flux and the q-coordinate chosen to be $90^{\circ}$ lagging. This transformation can be regarded as an extension of the Park's transformation.

Section 2.2 presents a space-vector pulse-width-modulation (PWM) technique for the control of a three-phase voltage-source inverter (VSI) that drives the induction 
motor. The space-vector PWM technique is used to generate the desired instantaneous reference voltages for the VSI from the corresponding basic space vectors according to the switching states.

Section 2.3 presents the field oriented control which provides an efficient real-time control of the torque of the induction motor which in terms controls the motor mechanical speed.

Section 2.4 presents the design of PID regulator used to regulate the motor torque and flux to the desired values.

\subsection{3-phase to 2-phase Transformation}

The three-phase voltages, currents and fluxes of an induction motor can be modeled in terms of complex space vectors. The space vector for the stator currents of the motor is defined as follows.

Assuming that $i_{a}, i_{b}, i_{c}$ are the three instantaneous currents in the stator, the complex stator current vector $i_{S}$ is defined by:

$$
\bar{i}_{S}=i_{a}+e^{j \frac{2}{3} \pi} i_{b}+e^{j \frac{4}{3} \pi} i_{c}
$$

This current space vector can be transformed into two currents: the d-coordinate current and the q-coordinate current by the following two steps:

Step 1: Transform the three-phase system into two orthogonal coordinate system. This transformation is known as $\alpha-\beta$ transformation or Clarke's transformation.

Step 2: Transform the $\alpha-\beta$ coordinates into the $d$-q coordinates, with the $d$-coordinate chosen to be in line with the rotor flux and q-coordinates chosen to be $90^{\circ}$ lagging. This transformation can be regarded as an extension of the Park's transformation.

\subsection{1 $\alpha-\beta$ Transformation (Clarke's Transformation)}

The current space vector can be transformed into two orthogonal axes named $\alpha$ and $\beta$. The $\alpha$-coordinate is chosen in line with the phase " $a$ ", and the $\beta$-coordinate is $90^{\circ}$ lagging. Then the $\alpha-\beta$ coordinate currents and the three phase instantaneous currents are related as follows:

$$
\begin{aligned}
& i_{s \alpha}=i_{a} \\
& i_{s \beta}=\frac{1}{\sqrt{3}} i_{a}+\frac{2}{\sqrt{3}} i_{b}
\end{aligned}
$$


where $i_{a}+i_{b}+i_{c}=0$ is assumed. To further illustrate the above relationship, assume the three phase currents are balanced as given below:

$$
\begin{aligned}
& i_{a}=I \sin (\omega t) \\
& i_{b}=I \sin (\omega t+2 \pi / 3) \\
& i_{c}=I \sin (\omega t-2 \pi / 3)
\end{aligned}
$$

Then the $\alpha-\beta$ coordinate current becomes:

$$
\begin{aligned}
& i_{S \alpha}=I \sin (\omega t) \\
& i_{S \beta}=I \sin (\omega t+\pi / 2)
\end{aligned}
$$

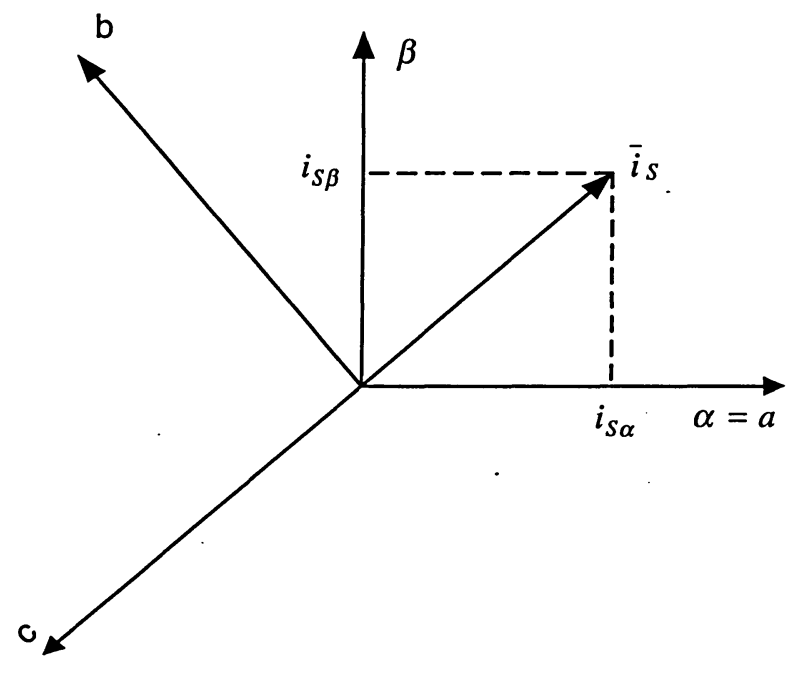

Figure 3: $\alpha-\beta$ Transformation

\subsection{2 d-q Transformation (Park's Transformation)}

The core transformation in the field oriented control (FOC) is the d-q transformation, which can be regarded as an extension of the Park's transformation. The $\alpha-\beta$ coordinates are transformed into the $d-q$ coordinates, with the d-coordinate chosen to be in line with the rotor flux $\Psi_{R}$ and the q-coordinate chosen to be $90^{\circ}$ lagging. Figure 4 shows the relationship between the current vectors in the two reference frames. 


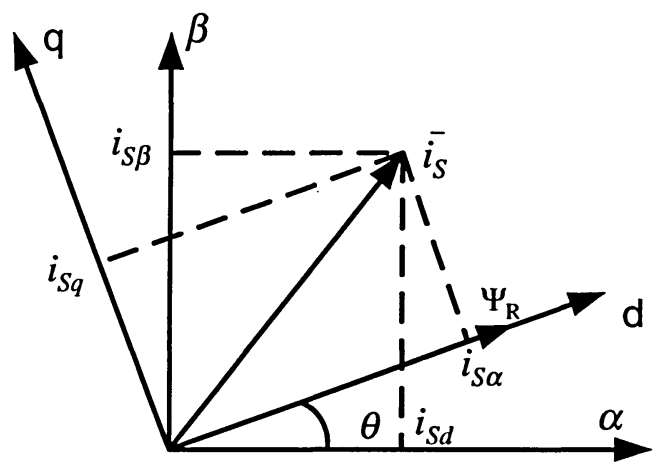

Figure 4: Park Transformation

In Figure 4, $\theta$ is the rotor flux angle. The $d$ and $q$ current components are determined by the following equations:

$$
\begin{aligned}
& i_{S d}=i_{S \alpha} \cos \theta+i_{s \beta} \sin \theta \\
& i_{S q}=-i_{S \alpha} \sin \theta+i_{s \beta} \cos \theta
\end{aligned}
$$

The d-q coordinate system has the following characteristics:

- It is a two-coordinate time-invariant system; both $i_{s d}$ and $i_{s q}$ are dc quantities.

- The current $i_{s d}$ is a flux related component and the current $i_{s q}$ is a torque related component.

- The torque of the motor can be easily controlled using these dc current data.

\subsection{Space Vector PWM Control of Motor Drive Inverter}

Space-vector pulse-width-modulation (PWM) technique has become a popular PWM technique for the control of three-phase voltage-source inverters (VSI) for applications such as induction motor drives. In comparison to the direct sinusoidal PWM technique, the space-vector PWM technique generates less harmonic distortion in the output voltages and currents and provides more efficient use of the dc supply voltage to the inverter [3].

Figure 5 shows a basic three-phase power inverter circuit, where $V a, V b, V c$ are the voltages applied to the induction motor, and where $V d c$ is the inverter dc input voltage. 


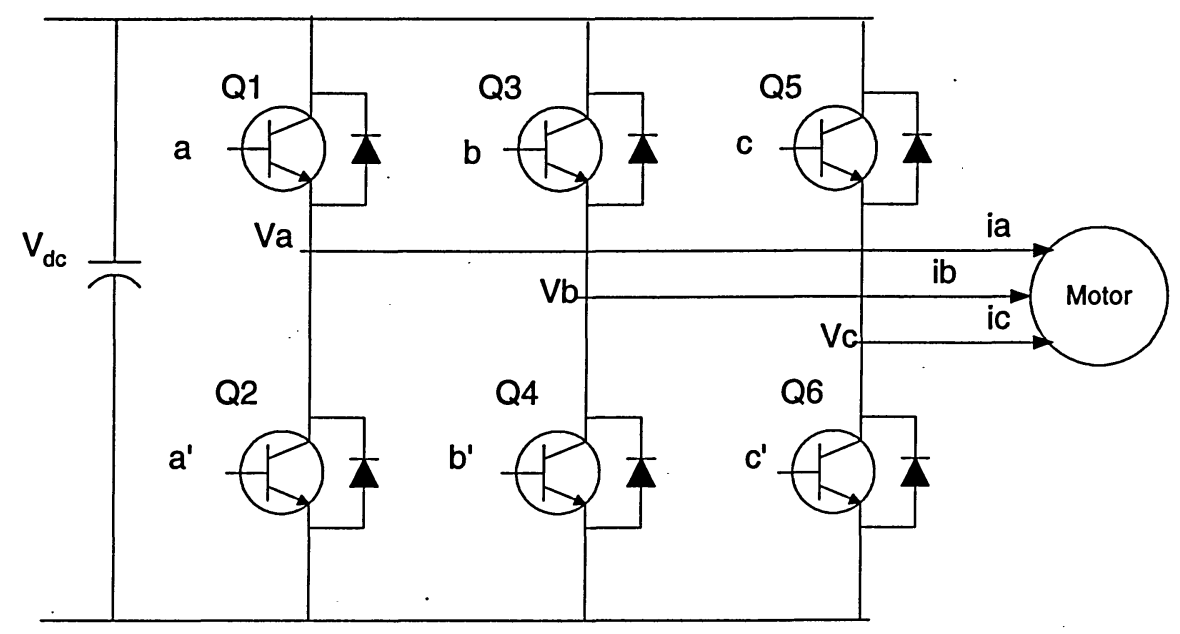

Figure 5: Three-phase Voltage Source Inverter

The six switches $Q 1$ to $Q 6$ are insulated-gate-bipolar-transistors (IGBT). The ON-OFF sequence of these switches follows the conditions below:

- Three of the switches must be always ON and three are always OFF.

- The upper and the lower switches of the same leg are driven with two complementary pulsed signals with dead band between the two signals to avoid short-circuit.

The induction motor is supplied with the required three phase voltages for the designed operating conditions using the PWM technique. In this research paper, the space-vector PWM method is used to generate the gating signals for the switches in the VSI inverter that drives the induction motor with high performance in terms of fast response to changes of loads and speed commands.

The relationship between the switching variable vector $\left[\begin{array}{lll}a & b & c\end{array}\right]^{T}$ and the line-to-line output voltage vector $\left[\begin{array}{llll}V_{a b} & V_{b c} & V_{c a}\end{array}\right]^{T}$ and the phase voltage vector $\left[\begin{array}{lll}V_{a} & V_{b} & V_{c}\end{array}\right]^{T}$ is given by the following equations.

$$
\begin{aligned}
& {\left[\begin{array}{l}
V_{a b} \\
V_{b c} \\
V_{c a}
\end{array}\right]=V_{d c}\left[\begin{array}{ccc}
1 & -1 & 0 \\
0 & 1 & -1 \\
-1 & 0 & 1
\end{array}\right]\left[\begin{array}{l}
a \\
b \\
c
\end{array}\right] .} \\
& {\left[\begin{array}{l}
V_{a} \\
V_{b} \\
V_{c}
\end{array}\right]=\frac{1}{3} V_{d c}\left[\begin{array}{ccc}
2 & -1 & 1 \\
-1 & 2 & -1 \\
-1 & -1 & 2
\end{array}\right]\left[\begin{array}{l}
a \\
b \\
c
\end{array}\right]}
\end{aligned}
$$

where $V_{a b}=V_{a}-V_{b}, V_{b c}=V_{b}-V_{c}, V_{c a}=V_{c^{-}} V_{a}$. The stator $\alpha-\beta$ voltages corresponding to the three-phase voltages are: 


$$
\begin{aligned}
& V_{S \alpha}=V_{a} \\
& V_{S \beta}=\frac{1}{\sqrt{3}} V_{a}+\frac{2}{\sqrt{3}} V_{b}
\end{aligned}
$$

The above equation can also be expressed in matrix form by using the equation $V_{a}+V_{b}+V_{c}=0$.

$$
\left[\begin{array}{l}
V_{s \alpha} \\
V_{s \beta}
\end{array}\right]=\frac{2}{3}\left[\begin{array}{rrr}
1 & -\frac{1}{2} & -\frac{1}{2} \\
0 & \frac{\sqrt{3}}{2} & -\frac{\sqrt{3}}{2}
\end{array}\right]\left[\begin{array}{l}
V_{a} \\
V_{b} \\
V_{c}
\end{array}\right]
$$

There are eight $\left(2^{3}\right)$ possible combinations for the switch commands. These eight switch combinations determine the eight phase voltage vectors, of which the results are six non-zero vectors $\left(V_{1}-V_{6}\right)$ and two zero vectors $\left(V_{0}, V_{7}\right)$ as shown in Figure 6.

The objective of space vector PWM technique is to generate the desired instantaneous reference voltages from the corresponding basic space vectors based on the switching states. Figure 6 shows that the basic space vectors divide the plan into six sectors. Depending on the sector that the reference voltage is in, two adjacent basic vectors are chosen. The two vectors are time weighted in a sample period $T$ (PWM period) to produce the desired output voltage.

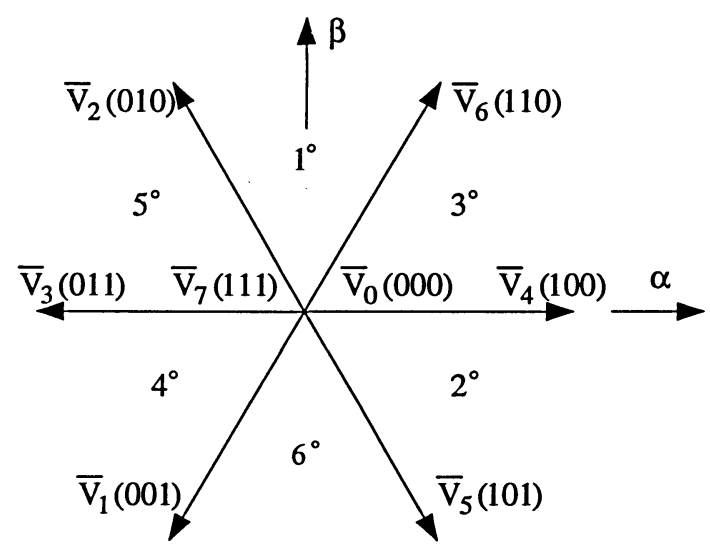

Figure 6: Configuration of Space Vector PWM

Assuming that the reference vector $V_{\text {out }}$ is in the sector 3 as shown in Figure $7 \mathrm{a}$, the application time of two adjacent vectors is given by:

$$
\begin{aligned}
& T=T_{4}+T_{6}+T_{0} \\
& \bar{V}_{\text {out }}=\frac{T_{4}}{T} \bar{V}_{4}+\frac{T_{6}}{T} \bar{V}_{6}
\end{aligned}
$$

where $T_{4}$ and $T_{6}$ are the durations of the basic vectors $V_{4}$ and $V_{6}$ to be applied respectively. $T_{0}$ is the duration for the zero vectors $\left(V_{0}\right.$ or $\left.V_{7}\right)$. Once the reference voltage $V_{\text {out }}$ and the PWM period $T$ are known, $T_{4}, T_{6}$ and $T_{0}$ can be determined according to the above equation [3]. 


$$
\begin{aligned}
& T_{4}=\frac{T}{2 V_{d c}}\left(3 V_{S \alpha}-\sqrt{3} V_{S \beta}\right) \\
& T_{6}=\sqrt{3} \frac{T}{V_{d c}} V_{s \beta} \\
& T_{0}=T-\left(T_{4}+T_{6}\right)
\end{aligned}
$$

Where $V_{S \alpha}, V_{S \beta}$ are $\alpha-\beta$ components of $V_{\text {out }}$. The voltage $V_{\text {out }}$ is an approximation of the desired output voltage based on the assumption that the change of output voltage is negligible within a PWM period $T$. Therefore it is critical that the PWM period is small with respect to the change of $V_{\text {out }}$. In practice, the approximation is very good because the calculation is

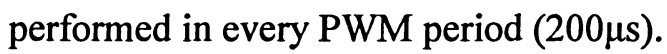

Figure 7b shows the pattern of space vector PWM in Sector 3. The space vector PWM is implemented in the DSP TMS320LF2407 in Ryerson Power Electronic Laboratory, where $t_{a o n}, t_{b o n}$ and $t_{c o n}$ are the ON durations of switches $Q 1, Q 3$ and $Q 5$ respectively. CMPR1, CMPR2 and CMPR3 are internal compare registers of DSP used to implement symmetrical PWM waveforms. Similarly, the patterns of the space vector PWM in other sectors can be formulated.

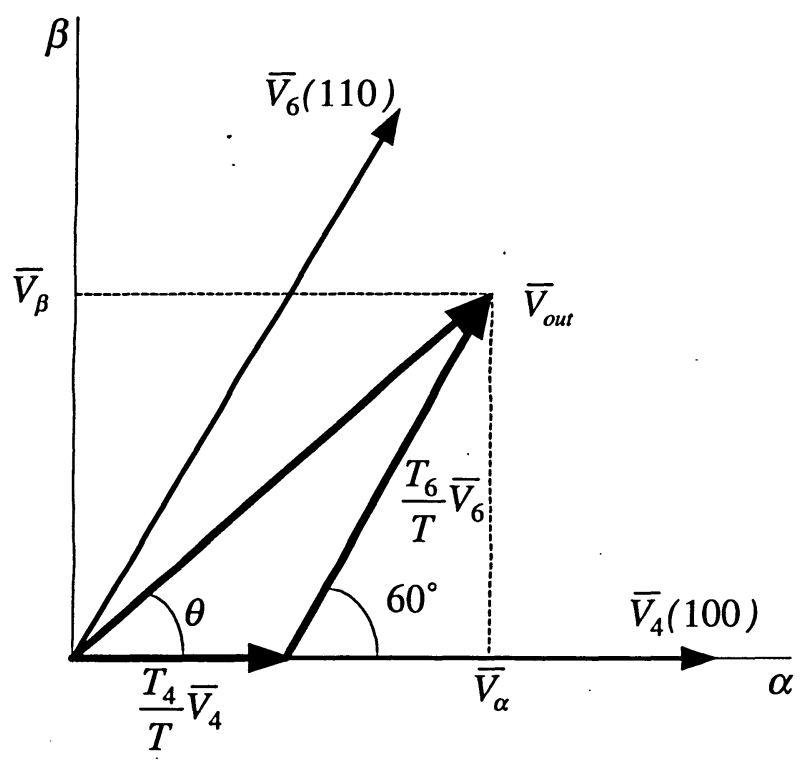

(a) Reference Voltage and Its Projections 


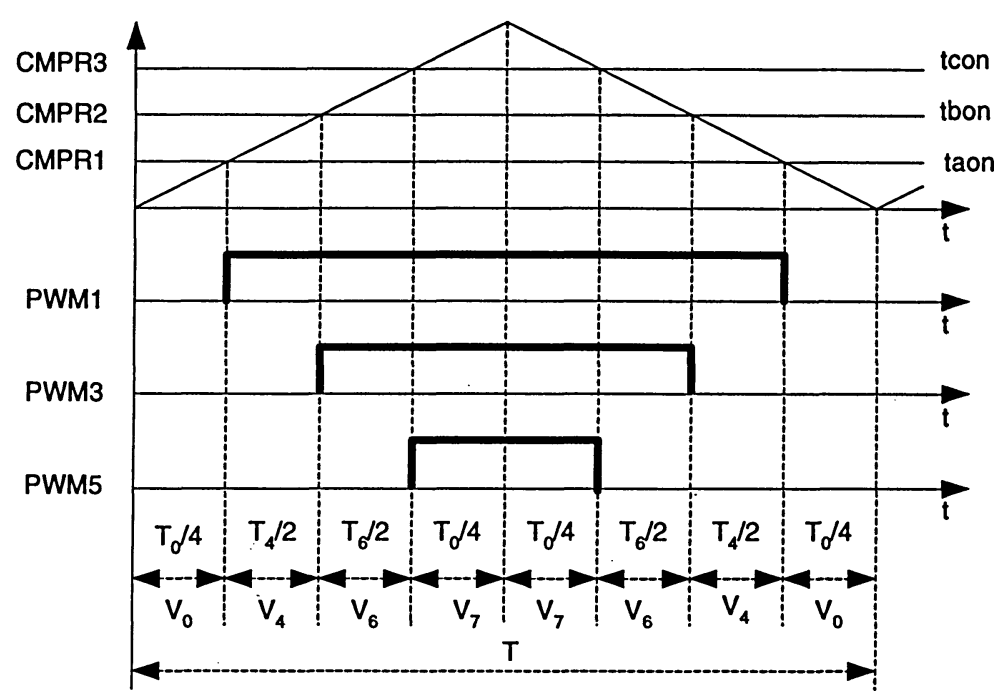

(b) PWM Pattern in Sector 3

Figure 7: Example of Space Vector PWM Pattern

\subsection{Field Oriented Control}

The field oriented control (FOC) designed in this research paper provides an efficient real-time control of the torque of the induction motor, which in terms controls the motor mechanical speed. The FOC also regulates the phase currents to avoid any current surges during the motor transient operation. The following describes the FOC method.

\subsubsection{The Basic FOC Scheme}

Figure 8 shows the basic scheme for induction motors using the FOC method. In this control scheme, two motor input currents $i_{a}, i_{b}$ are measured. These measurements are fed into the $\alpha-\beta$ transformation module. The outputs of this module are the two $\alpha-\beta$ currents: $i_{s \alpha}$ and $i_{s \beta}$. They are inputs to the d-q transformation module. This module computes the two currents $i_{s d}$ and $i_{s q}$ in the d-q rotating reference frame. Then $i_{s d}$ and $i_{s q}$ (they are virtually dc quantities) are compared to the flux reference $i_{\text {sdref }}$ and the torque reference $i_{\text {sqref. }}$. The torque reference is the output of the speed regulator in the speed feedback control module. The outputs of the two PID regulators are $V_{s d}$ and $V_{s q}$. They become the inputs to the inverse d-q transformation module. The outputs of this module are $V_{s \alpha}$ and $V_{s \beta}$, which are the stator vector voltages in the $\alpha-\beta$ orthogonal reference frame. They are the inputs to the space vector PWM control module. The outputs of this module are the switching signals that operate the voltage source inverter (VSI) for driving the induction motor. Note that both d-q transformation and its inverse transformation require the value of the rotor flux 
position for their computation. Therefore the rotor flux position needs to be determined prior to the transformation calculations.

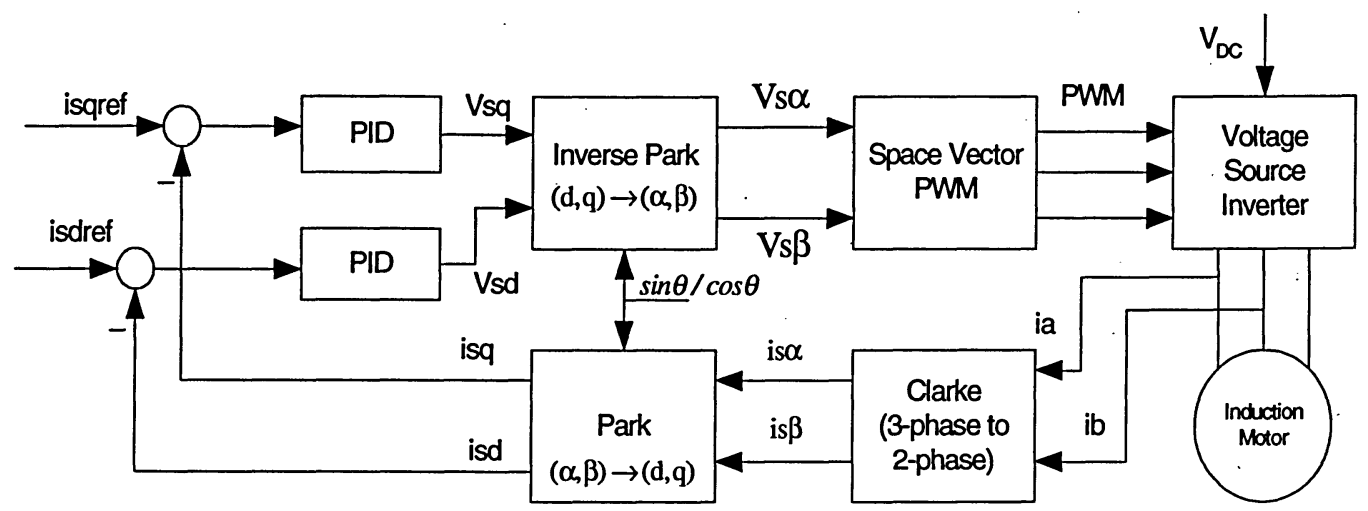

Figure 8: Field Oriented Control of Induction Motors

\subsubsection{Rotor Flux Position}

The information of the rotor flux position is crucial to the FOC control. Figure 9 shows the three reference frames, and the rotor flux position, the stator current space vector and the voltage space vector which rotate with respect to the $d-q$ reference at the synchronous speed.

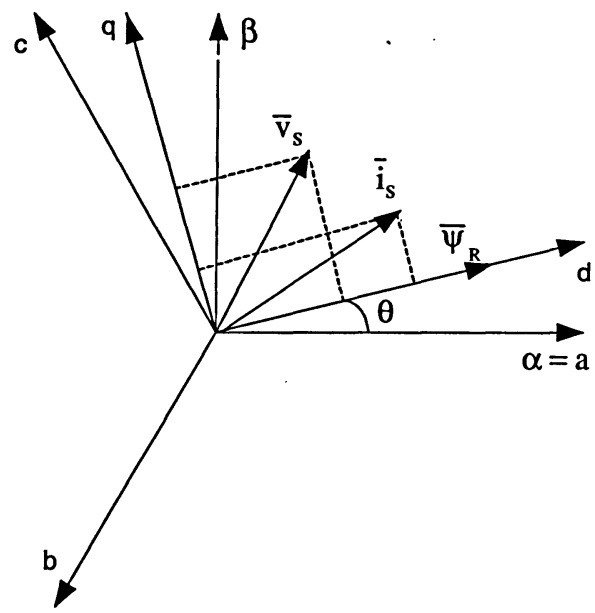

Figure 9: Relationship between Current, Voltage and Rotor Flux

In the induction motor, the speed of rotor is less than the speed of the rotor flux because of the slip due to friction and the mechanical load. For the FOC control, a specific method is needed to calculate $\theta$. The basic method is the use of the current model that needs two equations of the motor model in $d-q$ reference frame and a speed sensor [1]. Another method is to use a statistically optimal observer named the Kalman filter, to estimate the speed for achieving a sensorless control of induction motors. The details of this method is 
presented in Chapter 3. Figure 10 shows the block diagram of the sensorless control. This research paper is focused on the development of this control method.

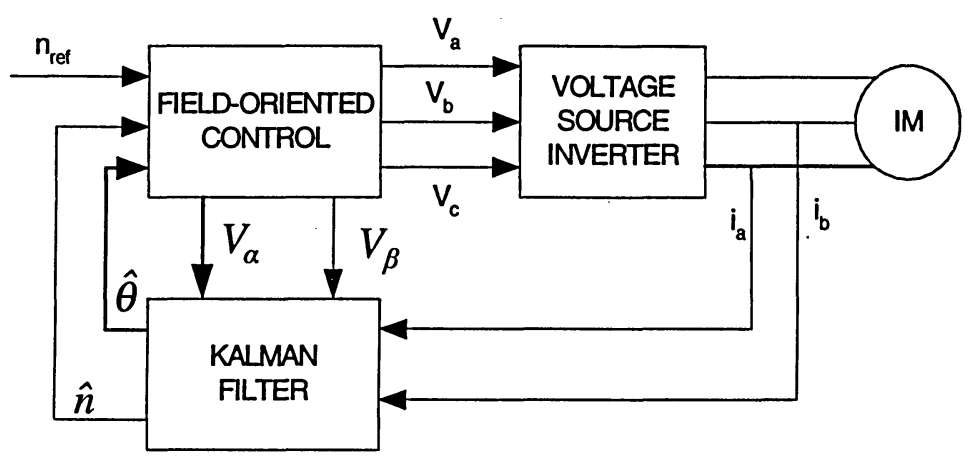

Figure 10: Sensorless Controller for Induction Motors

\subsection{Adaptive PID Regulator}

A motor drive based on the field-oriented control needs two inputs: the torque component reference $i_{\text {sqref }}$ and the flux component reference $i_{\text {sdref. }}$. The classical proportional and integral (PI) regulator is often used to regulate the motor torque and flux to the desired values. This regulator which is implemented in this research paper, is capable of reaching constant references by correctly setting both the $\mathrm{P}$ term $\left(K_{P}\right)$ and the $\mathrm{I}$ term $\left(K_{I}\right)$. The $\mathrm{P}$ term and $\mathrm{I}$ term respectively regulate the error sensibility and the steady state error. The regulation can be improved with the adaptive proportional-integral-derivative (PID) regulator [14].

To design a digital PID controller for the motor control, it may first consider the transfer function of an analog PID regulator:

$$
D(s)=K_{P}+K_{I} \frac{1}{s}+K_{D} s
$$

where $K_{P}$ is the proportional gain, $K_{I}$ is the integral gain, and $K_{D}$ is the derivative gain. Similar to the Laplace Transform in continuous time domain, the integrator and differentiator can be represented by pulse transfer function in discrete domain.

$$
\begin{aligned}
& \text { Integrator }=\frac{T(z+1)}{2(z-1)} \\
& \text { Differentiator }=\frac{z-1}{T z}
\end{aligned}
$$

where $T$ is the sampling period. Thus the transfer function of a digital non-adaptive PID controller is

$$
\begin{aligned}
D(z)=K_{P} & +K_{I} \frac{T(z+1)}{2(z-1)}+K_{D} \frac{z-1}{T z} \\
& =\frac{a_{0}+a_{1} z^{-1}+a_{2} z^{-2}}{1-z^{-1}}
\end{aligned}
$$


where

$$
\begin{aligned}
& a_{0}=K_{P}+\frac{K_{I} T}{2}+\frac{K_{D}}{T} \\
& a_{1}=-K_{P}+\frac{K_{I} T}{2}-\frac{2 K_{D}}{T} \\
& a_{2}=\frac{K_{D}}{T}
\end{aligned}
$$

Figure 11 shows the block diagram of an adaptive control system. In this figure, $r$ is the input or set point, $c$ is the output feedback, $y$ is the output and $\mathrm{D}(\mathrm{z})$ is the adaptive PID controller. The adaptive control scheme consists of two parts. First, the regulator uses initial (or updated) PID parameters and feedback input samples to determine the regulation. Second, the regulator updates the PID parameters until the error signal $e_{2}$ is approaching zero.

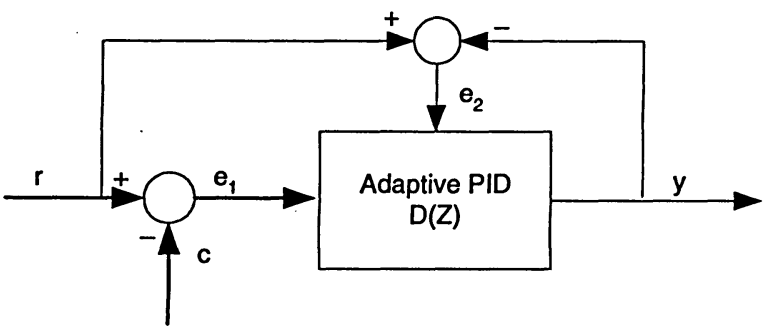

Figure 11: Adaptive PID Control

A quadratic objective function is used to minimize $e_{2}$ with respect to the regulator parameters.

$$
\begin{aligned}
f\left(a_{0}, a_{1}, a_{2}\right) & =\frac{1}{2}\left(e_{2}\right)^{2} \\
& =\frac{1}{2}[r(z)-y(z)]^{2} \\
& =\frac{1}{2}\left[r(z)-\left(\frac{a_{0}+a_{1} z^{-1}+a_{2} z^{-2}}{1-z^{-1}}\right) e_{1}\right]^{2}
\end{aligned}
$$

The first order partial derivatives with respect to the regulator parameter $a_{0}, a_{1}, a_{2}$ are given below:

$$
\frac{\partial f}{\partial a_{0}}=-r\left(\frac{1}{1-z^{-1}}\right) e_{1}+\left(\frac{a_{0}+a_{1} z^{-1}+a_{2} z^{-2}}{1-z^{-1}}\right)\left(\frac{1}{1-z^{-1}}\right) e_{1}^{2}
$$




$$
\begin{aligned}
& \frac{\partial f}{\partial a_{1}}=-r\left(\frac{z^{-1}}{1-z^{-1}}\right) e_{1}+\left(\frac{a_{0}+a_{1} z^{-1}+a_{2} z^{-2}}{1-z^{-1}}\right)\left(\frac{z^{-1}}{1-z^{-1}}\right) e_{1}^{2} \\
& \frac{\partial f}{\partial a_{2}}=-r\left(\frac{z^{-2}}{1-z^{-1}}\right) e_{1}+\left(\frac{a_{0}+a_{1} z^{-1}+a_{2} z^{-2}}{1-z^{-1}}\right)\left(\frac{z^{-2}}{1-z^{-1}}\right) e_{1}^{2}
\end{aligned}
$$

where $a_{0}, a_{1}$ and $a_{2}$ can be solved according to the steepest decent method of the gradient techniques, so that the following equations can be obtained.

$$
a_{n}(k+1)=a_{n}(k)+\beta \frac{\partial f}{\partial a_{n}} \quad n=0,1,2 ; \quad k=0,1,2 \cdots
$$

where $\beta$ is the parameter along the search direction. When combining equations (20) and (21) and $r=e_{2}+y=e_{2}+D(z) e_{1}$, the following equations can be obtained.

$$
\begin{aligned}
\frac{\partial f}{\partial a_{0}} & \approx-e_{2}\left(\frac{1}{1-z^{-1}}\right) e_{1} \\
\frac{\partial f}{\partial a_{1}} & \approx-e_{2}\left(\frac{z^{-1}}{1-z^{-1}}\right) e_{1} \\
\frac{\partial f}{\partial a_{2}} & \approx-e_{2}\left(\frac{z^{-2}}{1-z^{-1}}\right) e_{1}
\end{aligned}
$$

If ignoring the second order parts in $\partial f / \partial a_{n}$, the negative gradient is basically the product of error signal $e_{2}$ and error signal $e_{1}$ when using the first order approximation. Therefore, a modified gradient method is used as the search direction in updating the PID parameters. This also agrees with the general adaptive mechanism mentioned in [15]:

new parameter $=$ old parameter +

(bounded step size) $\times($ function of input $) \times$ (function of error).

More specifically equation (21) can be written into:

$$
a_{n}(k+1)=a_{n}+\beta e_{2}(k) e_{1}(k-n) \quad n=0,1,2 ; k=0,1,2 \cdots
$$




\section{Chapter 3}

\section{Kalman Filter}

\section{for Speed Senforless Control of Induction Motors}

This chapter presents the design of a control observer named the Kalman filter for efficient induction motor control which does not require any speed / position sensors. The Kalman filter is an optimal recursive algorithm, which provides the minimum variance state estimation for a time-varying linear system. It can tolerate system modeling and measurement errors, which are considered as noise processes in the state estimation. The Kalman filter processes all available measurements regardless of their precision, and provides a quick and optimal estimate of the variables of interest, as well as achieves a fast convergence. Its extension to applications for non-linear systems is called the Extended Kalman Filter (EKF). This chapter describes the Kalman filter and its application to induction motor drives.

Section 3.1 presents the basics for Kalman filter. First, the basic formulation of the Kalman filter control for the linear systems is provided. Second, the Kalman filter is extended for the nonlinear systems, in particular for the induction motor control systems. Third, an efficient recursive algorithm for the DSP implementation of the Kalman filter is provided.

Section 3.2 presents the specific formulation of induction motors for the implementation of Kalman filter in the speed sensorless field-oriented control. First, the d-q model of the induction motor is re-formulated such that the rotor flux linkage and speed can be computed efficiently. Second, the per unit formulation is provided for the implementation in the fixed-point DSPs.

\subsection{Basics of Kalman Filter}

Assume a linear system with the following equations.

$$
\begin{array}{ll}
\text { State Equation: } & \dot{x}=A x+B u+w \\
\text { Output Equation: } & y=C x+v
\end{array}
$$

where $x$ is the state variable vector, $y$ is the output vector, $u$ is the input vector, and $A, B$, $C$ are the system matrices. $\quad w$ and $v$ are the system and the measurement noises respectively.

Assuming that $w$ and $v$ are stationary, white, uncorrelated and Gauss noises, their 
expectations are zero, their covariance matrices are $Q$ and $R$ respectively.

$$
\begin{aligned}
& \operatorname{cov}(w)=E\left\{w w^{T}\right\}=Q \\
& \operatorname{cov}(v)=E\left\{v v^{T}\right\}=R
\end{aligned}
$$

where $E\{$.$\} denotes the expected value, that is:$

$$
E(w)=\int_{-\infty}^{\infty} w f(w) d w
$$

where $f(w)$ is the probability of $w$.

Briefly speaking, Kalman filter has the same structure as an observer shown in Figure 12 . The system equation of the Kalman filter is as follows.

$$
\dot{\hat{x}}=A \hat{x}+B u+K(y-C \hat{x})=(A-K C) \hat{x}+B u+K y
$$

where $\hat{x}$ is the estimate of state $x$. Kalman gain matrix $\mathrm{K}$ is a solution based on the well-known Riccati equation, which is developed to minimize the following quadratic objective function [6].

$$
\text { Minimize: } \quad J=\sum_{i=1}^{n} E\left\{e_{i}^{2}\right\}, \quad e=x-\hat{x}
$$

Kalman gain: $\quad K=P C^{T} R^{-1}$

$$
\text { Riccati equation: } \quad A P+P A^{T}-P C^{T} R^{-1} C P+Q=0
$$

The error covariance matrix $P$ is the solution of Riccati equation, which is used to determine the feedback matrix $K$ of the Kalman filter. The matrix $K$, which is called Kalman gain, determines how the estimate of the state vector $\hat{x}$ is modified after the output of Kalman filter is compared with the real output of the system.

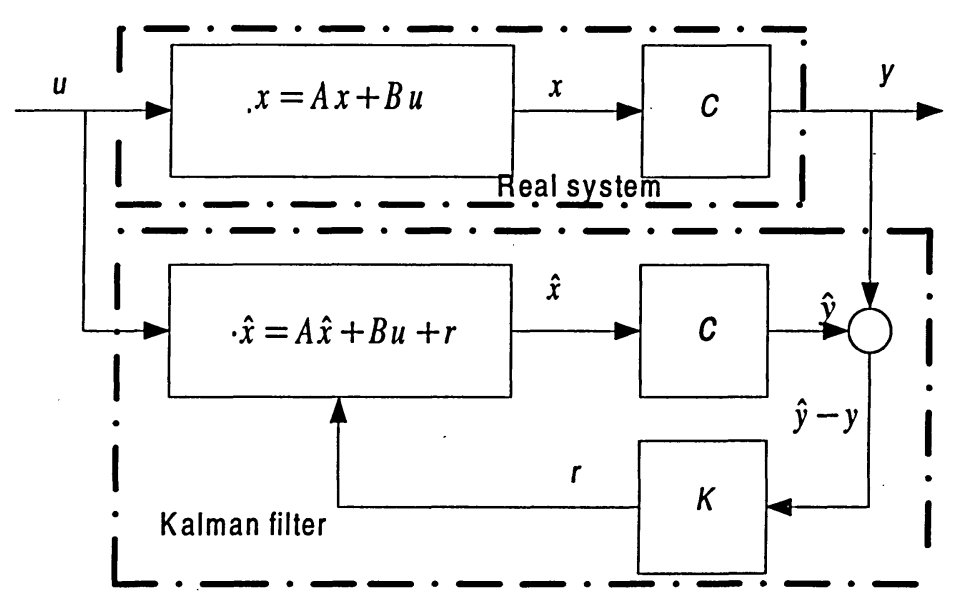

Figure 12: Principle of Kalman Filter 
The extended Kalman filter (EKF) is developed to deal with the non-linearity of the induction motor. Assume $g$ and $h$ are the functions of the state equation and the output equation for a nonlinear system.

$$
\begin{gathered}
\dot{x}=g(x, u, t) \\
y=h(x)
\end{gathered}
$$

The discrete time model of the nonlinear system can be represented in the following equations.

$$
\begin{aligned}
& x(k+1)=x(k)+T g(x(k), u(k), k)=\Phi(x(k), u(k), k) \\
& y(k)=h(x(k))
\end{aligned}
$$

Where $T$ is the sample period. The linearizing model of equation (33) is as follows.

$$
\begin{aligned}
& x(k+1)=A(k) x(k)+B(k) U(k)+w(k) \\
& y(k)=C(k) x(k)+v(k)
\end{aligned}
$$

where

$$
A(k)=\left.\frac{\partial \Phi}{\partial x}\right|_{x=x_{k}}, \quad B(k)=\left.\frac{\partial \Phi}{\partial u}\right|_{u=u_{k}}, \quad C(k)=\left.\frac{\partial h}{\partial x}\right|_{x=x_{k}}
$$

The $v(k)$ and $w(k)$ are considered as noises, with their covariance matrices $R$ and $Q$. If the input $U_{k}$ is perfectly known, then the prediction of state $x_{k}$ is as follows:

$$
\hat{x}(k+1)=A(k) \hat{x}(k)+B(k) U(k)
$$

The state prediction error is found by subtracting equations (36) from equation (34) as given below.

$$
\hat{x}(k+1)-x(k+1)=A(k)[\hat{x}(k)-x(k)]-w(k)
$$

For simplicity, equation (37) can be written as:

$$
\begin{aligned}
& \hat{x}_{k+1}-x_{k+1}=A_{k}\left(\hat{x}_{k}-x_{k}\right)-w_{k} \\
& e_{k+1}=A_{k} e_{k}-w_{k} \\
& e_{k}=\hat{x}_{k}-x_{k}
\end{aligned}
$$

Hence, the covariance matrix $P_{k}$ of state prediction error $e_{k}$ is:

$$
P_{k+1}=A_{k} P_{k} A_{k}{ }^{T}+Q
$$

The objective function for estimating $x_{k+1}$ is set as follows.

$$
\hat{x}_{k+1}=\arg \min _{x}\left[\left(\hat{x}_{k}-x\right)^{T} P_{k}^{-1}\left(\hat{x}_{k}-x\right)+\left(y_{k}-C_{k} x\right)^{T} R^{-1}\left(y_{k}-C_{k} x\right)\right]
$$

The minimum is found by putting the partial derivative with respect to $x$ equal to zero. This yield:

$$
\begin{gathered}
\hat{x}_{k+1}=\hat{x}_{k}+K_{k}\left(y_{k}-C_{k} \hat{x}_{k}\right) \\
P_{k+1}=P_{k}-K_{k} C_{k} P_{k}
\end{gathered}
$$




$$
K_{k}=P_{k} C_{k}^{T}\left(R+C_{k} P_{k} C_{k}{ }^{T}\right)^{-1}
$$

The extended Kalman filter (EKF) can be implemented in a DSP using a recursive algorithm given below. The implementation requires the following five steps:

1) State vector prediction at instant $k+1$.

$$
x_{k+1 / k}=\Phi\left(x_{k / k}, u_{k}, k\right)
$$

2) Prediction error covariance matrix computation.

$$
P_{k+1 / k}=A_{k} P_{k / k} A_{k}^{T}+Q
$$

3) Kalman gain computation at instant $k+1$.

$$
K_{k+1}=P_{k+1 / k} C_{k}^{T}\left(C_{k} P_{k+1 / k} C_{k}^{T}+R\right)^{-1}
$$

4) State vector estimation at instant $k+1$.

$$
x_{k+1 / k+1}=x_{k+1 / k}+K_{k+1}\left(y_{k+1}-h\left(x_{k+1 / k}\right)\right)
$$

5) Filter error covariance matrix computation at instant $k+1$.

$$
P_{k+1 / k+1}=P_{k+1 / k}-K_{k+1} C_{k} P_{k+1 / k}
$$

Equation (47) is crucial to the EKF algorithm, because it provides the state estimation. Figure 13 shows the EKF algorithm used in induction motor control.

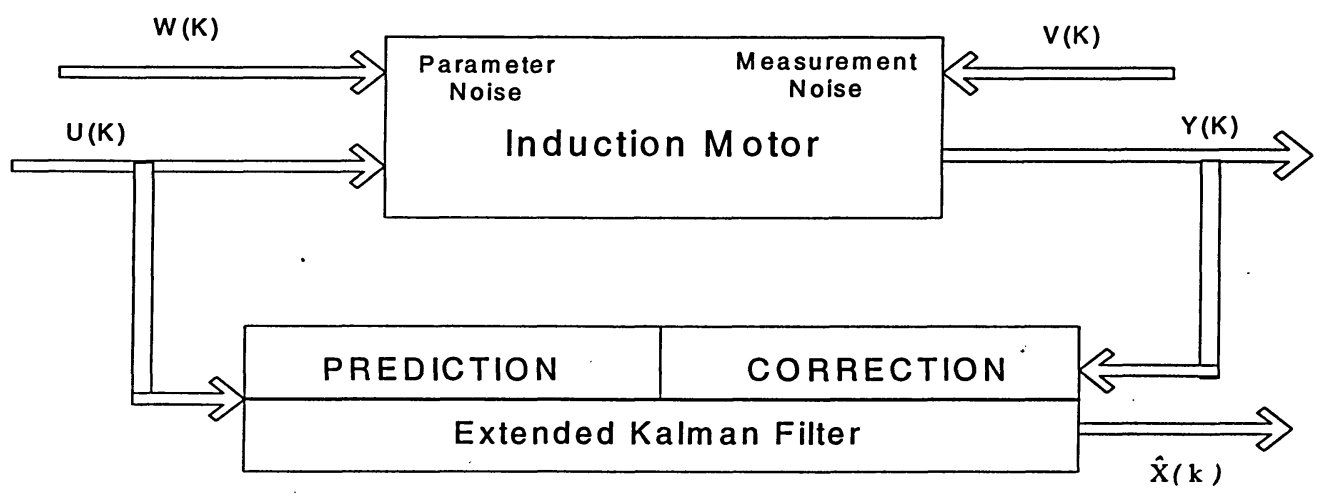

. Figure 13: Extended Kalman Filter Algorithm

\subsection{Induction Motor Model for Implementation of Kalman Filter}

As shown in previous section, an appropriate model of induction motor is required to 
implement the Kalman filter [16]. The following presents the formulation of induction motors for the implementation of the Kalman filter.

The stator and rotor flux in the stator reference frame are defined as

$$
\begin{aligned}
& \Psi_{S \alpha}=L_{S} i_{S \alpha}+L_{H} i_{R \alpha} \\
& \Psi_{S \beta}=L_{S} i_{S \beta}+L_{H} i_{R \beta} \\
& \Psi_{R \alpha}=L_{R} i_{R \alpha}+L_{H} i_{S \alpha} \\
& \Psi_{R \beta}=L_{R} i_{R \beta}+L_{H} i_{S \beta}
\end{aligned}
$$

The $\alpha-\beta$ stator voltages in the stator reference frame are:

$$
\begin{aligned}
& V_{S \alpha}=R_{S} i_{S \alpha}+\frac{d}{d t} \Psi_{S \alpha} \\
& V_{S \beta}=R_{S} i_{S \beta}+\frac{d}{d t} \Psi_{S \beta}
\end{aligned}
$$

The $\alpha-\beta$ rotor voltages in the stator reference frame are:

$$
\begin{aligned}
& V_{R \alpha}=R_{R} i_{R \alpha}+\omega \Psi_{R \beta}+\frac{d}{d t} \Psi_{R \alpha} \\
& V_{R \beta}=R_{R} i_{R \phi}-\omega \Psi_{R \alpha}+\frac{d}{d t} \Psi_{R \beta}
\end{aligned}
$$

For squirrel-cage induction motor, the rotor voltages are zero that is $V_{R \alpha}=0, V_{R \beta}=0$. Since the digital computation period for the speed control loop is very short as compared to the induction motor speed, the speed can be considered constant to have no change during one sampling period, that is $d \omega / d t=0$. If the required rotor flux $\Psi_{R \alpha}, \Psi_{R \beta}$, stator current $i_{s a}, i_{s \beta}$ and speed $\omega$ are selected as state variables, the state equation and output equation of the induction motor can be written as:

1) State equation is

$$
\frac{d}{d t}\left[\begin{array}{c}
i_{S \alpha} \\
i_{S \beta} \\
\Psi_{R \alpha} \\
\Psi_{R \beta} \\
\omega
\end{array}\right]=\left[\begin{array}{ccccc}
-\frac{K_{R}}{K_{L}} & 0 & \frac{L_{H} R_{R}}{L_{R}^{2} K_{L}} & \frac{L_{H} \omega}{L_{R} K_{L}} & 0 \\
0 & -\frac{K_{R}}{K_{L}} & -\frac{L_{H} \omega}{L_{R} K_{L}} & \frac{L_{H} R_{R}}{L_{R}^{2} K_{L}} & 0 \\
\frac{L_{H}}{T_{R}} & 0 & -\frac{1}{T_{R}} & -\omega & 0 \\
0 & \frac{L_{H}}{T_{R}} & \omega & -\frac{1}{T_{R}} & 0 \\
0 & 0 & 0 & 0 & 0
\end{array}\right]\left[\begin{array}{c}
i_{S \alpha} \\
i_{S \beta} \\
\Psi_{R \alpha} \\
\Psi_{R \beta} \\
\omega
\end{array}\right]+\frac{1}{K_{L}}\left[\begin{array}{ll}
1 & 0 \\
0 & 1 \\
0 & 0 \\
0 & 0 \\
0 & 0
\end{array}\right]\left[\begin{array}{c}
u_{S \alpha} \\
u_{S \beta}
\end{array}\right]
$$

2) Output equation is 


$$
\left[\begin{array}{c}
i_{S \alpha} \\
i_{S \beta}
\end{array}\right]=\left[\begin{array}{lllll}
1 & 0 & 0 & 0 & 0 \\
0 & 1 & 0 & 0 & 0
\end{array}\right]\left[\begin{array}{c}
i_{S \alpha} \\
i_{S \beta} \\
\Psi_{R \alpha} \\
\Psi_{R \beta} \\
\omega
\end{array}\right]
$$

where $K_{R}=R_{S}+\left(L_{H} / L_{R}\right)^{2} R_{R}, \quad K_{L}=\sigma L_{S}$. The per unit model is useful for the digital implementation as it converts various quantity ranges into one single range. This allows to choose one accurate numerical representation for the complete system. With the implementation in the fixed-point DSP TMS320LF2407, the $Q 15$ format is used in this research paper for its greatest precision. In per unit model, all quantities are scaled with respect to their base values.

$$
\begin{aligned}
& i_{S \alpha}=i_{S \alpha}^{n} I_{b} \quad \Psi_{R \alpha}=\Psi_{R \alpha}^{n} \Psi_{b} \quad u_{S \alpha}=u_{S \alpha}^{n} U_{b} \\
& i_{S \beta}=i_{S \beta}^{n} I_{b}, \quad \Psi_{R \beta}=\Psi_{R \beta}^{n} \Psi_{b}{ }^{\prime} \quad u_{S \beta}=u_{S \beta}^{n} U_{b}, \quad \omega=\omega^{n} \omega_{b} \\
& V_{b}=I_{b} Z_{b} \\
& \Psi_{b}=V_{b} / \omega_{b}
\end{aligned}
$$

where $I_{b}, V_{b}$ are the base values of the phase current and voltage. $\omega_{b}$ is the nominal speed, $\Psi_{b}$ is the base flux, $Z_{b}$ is the base impedance. $i_{s \alpha}^{n}, i_{s \beta}^{n}, \Psi_{R \alpha}^{n}, \Psi_{R \beta}^{n} u_{s \alpha}^{n}, u_{s \beta}^{n}, \omega^{n}$ are normalized currents, fluxes, voltages and speed respectively.

By using normalized values of state variables, equations (52) and (53) can be written as the Per Unit Model of Induction Motors below.

1) State equation is

$$
\frac{d}{d t}\left[\begin{array}{c}
i_{S \alpha}^{n} \\
i_{S \beta}^{n} \\
\Psi_{R \alpha}^{n} \\
\Psi_{R \beta}^{n} \\
\omega^{n}
\end{array}\right]=\left[\begin{array}{ccccc}
-\frac{K_{R}}{K_{L}} & 0 & \frac{L_{H} R_{R}}{L_{R}^{2} K_{L}} \frac{Z_{b}}{\omega_{b}} & \frac{L_{H} Z_{b} \omega^{n}}{L_{R} K_{L}} & 0 \\
0 & -\frac{K_{R}}{K_{L}} & -\frac{L_{H} Z_{b} \omega^{n}}{L_{R} K_{L}} & \frac{L_{H} R_{R}}{L_{R}^{2} K_{L}} \frac{Z_{b}}{\omega_{b}} & 0 \\
\frac{L_{H}}{T_{b}} \frac{\omega_{b}}{Z_{b}} & 0 & -\frac{1}{T_{R}} & -\omega_{b} \omega^{n} & 0 \\
0 & \frac{L_{H}}{T_{R}} \frac{\omega_{b}}{Z_{b}} & \omega_{b} \omega^{n} & -\frac{1}{T_{R}} & 0 \\
0 & 0 & 0 & 0 & 0
\end{array}\right]\left[\begin{array}{c}
i_{S \alpha}^{n} \\
i_{S \beta}^{n} \\
\Psi_{R \alpha}^{n} \\
\Psi_{R \beta}^{n} \\
\omega^{n}
\end{array}\right]+\frac{Z_{b}}{K_{L}}\left[\begin{array}{ll}
1 & 0 \\
0 & 1 \\
0 & 0 \\
0 & 0 \\
0 & 0
\end{array}\right]\left[\begin{array}{c}
u_{S \alpha}^{n} \\
u_{S \beta}^{n}
\end{array}\right]
$$

2) Output equation is 


$$
\left[\begin{array}{c}
i_{S \alpha}^{n} \\
i_{S \beta}^{n}
\end{array}\right]=\left[\begin{array}{lllll}
1 & 0 & 0 & 0 & 0 \\
0 & 1 & 0 & 0 & 0
\end{array}\right]\left[\begin{array}{c}
i_{S \alpha}^{n} \\
i_{S \beta}^{n} \\
\Psi_{R \alpha}^{n} \\
\Psi_{R \beta}^{n} \\
\omega^{n}
\end{array}\right]
$$

Using the linearizing form of the induction motor model, the matrices and vectors that are needed for the EKF recursive algorithm [equations (44)-(48)] can be calculated.

$$
\begin{aligned}
& \Phi=\left[\begin{array}{c}
\left(1-T \frac{K_{R}}{K_{L}}\right) i_{S \alpha}^{n}+T \frac{L_{H} R_{R}}{L_{R}^{2} K_{L}} \frac{Z_{b}}{\omega_{b}} \Psi_{R \alpha}^{n}+T \frac{L_{H} Z_{b}}{L_{R} K_{L}} \omega^{n} \Psi_{R \beta}^{n}+T \frac{Z_{b}}{K_{L}} u_{S \alpha}^{n} \\
\left(1-T \frac{K_{R}}{K_{L}}\right) i_{S \beta}^{n}-T \frac{L_{H} Z_{b}}{L_{R} K_{L}} \omega^{n} \Psi_{R \alpha}^{n}+T \frac{L_{H} R_{R}}{L_{R}^{2} K_{L}} \frac{Z_{b}}{\omega_{b}} \Psi_{R \beta}^{n}+T \frac{Z_{b}}{K_{L}} u_{S \beta}^{n} \\
T \frac{L_{H}}{T_{R}} \frac{\omega_{b}}{Z_{b}} i_{S \alpha}^{n}+\left(1-\frac{T}{T_{R}}\right) \Psi_{R \alpha}^{n}-T \omega_{b} \omega^{n} \Psi_{R \beta}^{n} \\
T \frac{L_{H}}{T_{R}} \frac{\omega_{b}}{Z_{b}} i_{S \beta}^{n}+T \omega_{b} \omega^{n} \Psi_{R \alpha}^{n}+\left(1-\frac{T}{T_{R}}\right) \Psi_{R \beta}^{n} \\
\omega^{n}
\end{array}\right] \\
& h=C x=\left[\begin{array}{l}
i_{s \alpha}^{n} \\
i_{s \beta}^{n}
\end{array}\right] \\
& A=\frac{\partial \Phi}{\partial x}=\left[\begin{array}{ccccc}
1-T \frac{K_{R}}{K_{L}} & 0 & T \frac{L_{H} R_{R}}{L_{R}^{2} K_{L}} \frac{Z_{b}}{\omega_{b}} & T \frac{L_{H} Z_{b}}{L_{R} K_{L}} \omega^{n} & T \frac{L_{H} Z_{b}}{L_{R} K_{L}} \Psi_{R \beta}^{n} \\
0 & 1-T \frac{K_{R}}{K_{L}} & -T \frac{L_{H} Z_{b}}{L_{R} K_{L}} \omega^{n} & T \frac{L_{H} R_{R}}{L_{R}^{2} K_{L}} \frac{Z_{b}}{\omega_{b}} & -T \frac{L_{H} Z_{b}}{L_{R} K_{L}} \Psi_{R \alpha}^{n} \\
T \frac{L_{H}}{T_{R}} \frac{\omega_{b}}{Z_{b}} & 0 & 1-\frac{T}{T_{R}} & -T \omega_{b} \omega^{n} & -T \omega_{b} \Psi_{R \beta}^{n} \\
0 & T \frac{L_{H}}{T_{R}} \frac{\omega_{b}}{Z_{b}} & T \omega_{b} \omega^{n} & 1-\frac{T}{T_{R}} & T \omega_{b} \Psi_{R \alpha}^{n} \\
0 & 0 & 0 & 0 & 1
\end{array}\right] \\
& C=\frac{\partial h}{\partial x}=\left[\begin{array}{lllll}
1 & 0 & 0 & 0 & 0 \\
0 & 1 & 0 & 0 & 0
\end{array}\right]
\end{aligned}
$$

First, the matrices and vectors for the induction motor model are calculated. Second, the recursive algorithm for the extended Kalman filter is executed. Third, the rotor flux and the rotor speed are computed. 
The recursive Kalman filter algorithm can accurately determine the speed of the induction motor and the rotor flux position or angle. This allows an accurate control of the motor speed without the need of a speed or position sensor. However, the implementation of the Kalman filter is a fairly involved process. The model of the induction motor must be calculated in real time, which involves many matrix multiplications. The DSP with high computation capability is most suitable for this type of control process.

Appendix B lists all the internal parameters of the induction motor used in this research paper, which are needed for the calculations of equations (44) to (48). 


\section{Chapter 4}

\section{Digital Signal Processor Implementation of Speed Sensorless Field Oriented Control}

This chapter presents the digital signal processor (DSP) implementation of the field oriented control (FOC) of induction motors without any speed or position sensors. The hardware and the software implementation of the speed sensorless FOC system is detailed in this chapter. The following outlines the sections in this chapter.

Section 4.1 presents an overview of the DSP FOC operation. This section describes the key operations which include determination of the rotor speed and flux angle using Kalman filter, d-q transformation with respect to the rotor flux, speed and torque regulation, and generation of PWM control signals.

Section 4.2 presents the hardware implementation of the FOC system for induction motors. This section describes the power electronic inverter circuit, the DSP control circuit, and their interface used for the implementation.

Section 4.3 presents the software implementation of the FOC operation. This section describes the software modules used to implement the sensorless speed control of induction motors.

Section 4.4 presents the debugging of hardware and software. This section describes the six phases used in debugging the FOC software and hardware.

\subsection{Overview of DSP FOC Operations}

The fixed-point DSP TMS320LF2407 is the core of the control system designed in this research paper. The following provides an overview of the DSP FOC operations.

1) The DSP operates its analog-to-digital converter (ADC) to collect the instantaneous induction motor input currents measured by a current transducer at the motor terminal.

2) The DSP executes the recursive algorithm of the extended Kalman filter, using the measured motor currents, to compute the $\alpha$ and $\beta$ currents, the rotor speed and flux angle. 
3) The DSP carries out a specific d-q transformation with the d-coordinate chosen to be in line with the rotor flux, using the $\alpha-\beta$ currents and the flux angle, to compute the d-coordinate and q-coordinate currents.

4) The DSP executes three feedback regulators for the motor speed, the rotor torque, and the rotor flux, to determine the d-coordinate and the q-coordinate stator reference voltages.

5) The DSP carries out an inverse d-q transformation, using the d-q reference voltages, to compute the stator $\alpha$ and $\beta$ reference voltages.

6) The DSP executes the space vector pulse-width-modulation (PWM) module, using the $\alpha-\beta$ reference voltages, to compute the PWM control signals.

7) The DSP finally outputs the PWM control signals to the gating circuits of the power electronic inverter that drives the induction motor.

\subsection{Hardware Implementation of Field Oriented Control}

Figure 14 shows the block diagram of the hardware required to implement a sensorless speed control system for an induction motor. The DSP control system designed in this research paper consists of the following major hardware components:

1) DSP controller: Texas Instruments, eZdspTMS320LF2407 development kit.

2) Current sensors: Two LEM, 10A current transducers.

3) Power inverter: Semikron-SkiiP2, IGBT module.

4) Induction motor: Teco-Westinghouse, 3-Phase, $60 \mathrm{~Hz}$ induction motor.

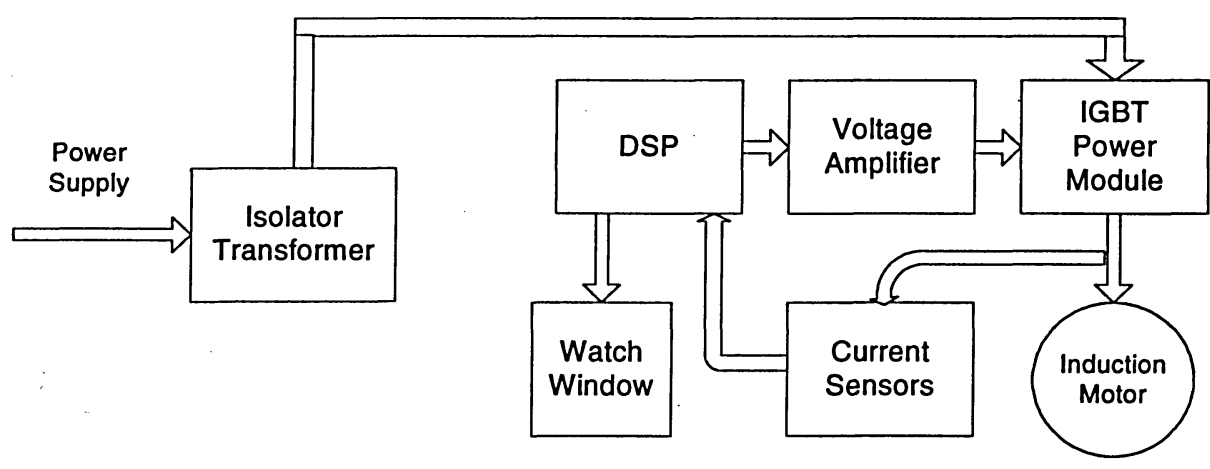

Figure 14. Experiment Setup for Sensorless Control System 
The power hardware used to implement and test the control scheme developed in this research paper is based on the six-power IGBT module (SkiiP2 from SEMIKRON). This module is controlled by a DSP (TMS320LF2407 from Texas Instruments) via a voltage-level shifter (MC14504). This shifter amplifies the PWM output voltages from $3.3 \mathrm{~V}$ to $15 \mathrm{~V}$ for gating the IGBT switches. The current sensing is performed via two current-to-voltage transducers ( $L E M$ type) supplied with $\pm 12 \mathrm{~V}$. Their maximum input current is $\pm 10 \mathrm{~A}$, which is converted into a $3.3 \mathrm{~V}$ output voltage to match the requirement of inputs to DSP. The induction motor is a 3-Phase, $60 \mathrm{~Hz}$ motor (EPACT from TECO-WESTINGHOUSE), of which the rated power is 5 horsepower.

DSP is the key control element in this research paper design of the FOC of induction motors. The settings of the DSP TMS320LF2407 are given as follows.

- Development/Emulation: Code Composer 4.1 supports real-time debugging.

- CPU Clock: 30MHz.

- PWM frequency: $5 \mathrm{kHz}$.

- PWM mode: Symmetrical with dead band $1.8 \mu \mathrm{s}$.

- Interrupts: 2 (T1 Underflow - System time base).

- System time base/PWM period: $200 \mu \mathrm{S}$.

- Peripheral Usage: Timer 1, PWM1-6, ADC (2 channels).

- Current loop sampling frequency: $5 \mathrm{kHz}$.

Two ADC input pins (Pin 4, 6 in port P1) are used to sense the two stator currents $i_{a}, i_{b}$. PWM 1-PWM6 output pins (Pin 9-14 in port P2) are used to drive the IGBT power inverter.

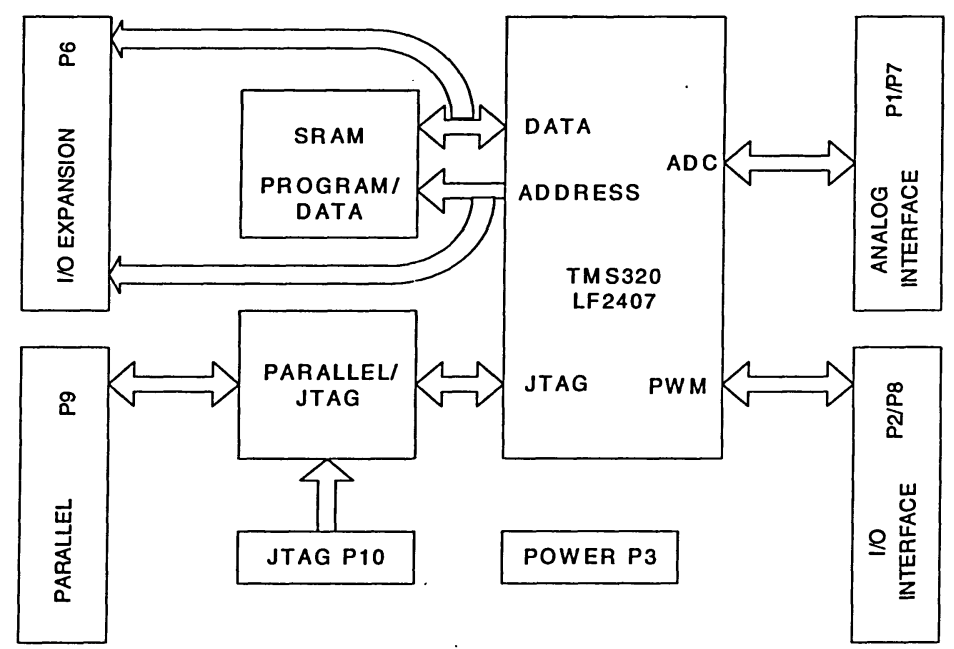

Figure 15: Block Diagram of eZdsp ${ }^{\mathrm{TM}}$ LF2407

The eZdsp LF2407 platform is used to develop and run software for the DSP TMS320LF2407. With 64K words of onboard program/data RAM, this platform allows a full speed verification of software code. To simplify code development and shorten 
debugging time, a C2000 Tools Code Composer driver is provided, which could run the software in real time. Figure 15 shows the block diagram of this platform.

\subsection{DSP Software Implementation of Field Oriented Control}

DSP control software developed in this research paper is based on two modules: the initialization and the run module. The initialization module is performed only once at the beginning of the software execution. The run module is based on a user interface loop interrupted by the PWM underflow. When this interrupt flag is set, the corresponding Interrupt Service Routine (ISR) is acknowledged and served. The complete extended Kalman filter (EKF) and the FOC algorithms are computed within a PWM interrupt at the same period as the chopping frequency $5 \mathrm{KHz}$. The overview of the software is given in Figure 16.
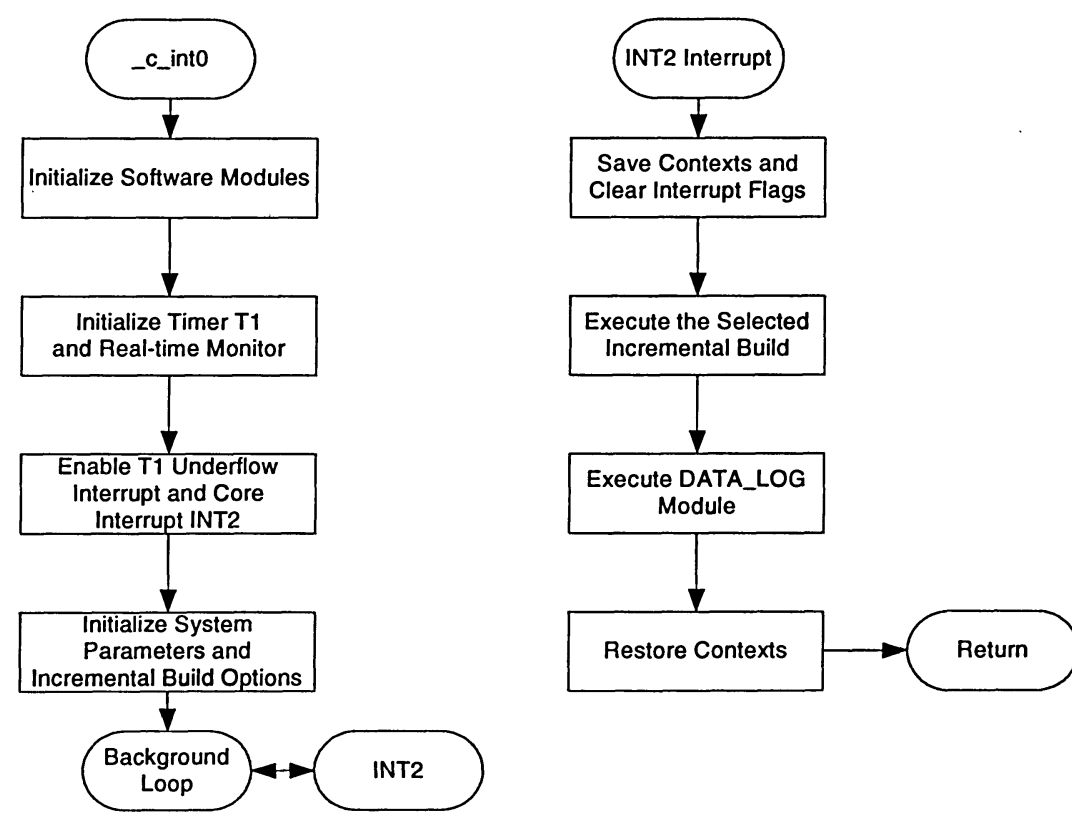

Figure 16: Software Flowchart

The DSP Controller Full Compare Units are designed to generate symmetrical complementary PWM signals to the power inverter at a frequency of $5 \mathrm{KHz}$. TIMER1 is used as the time base with a sampling period $\mathrm{T}=200 \mu \mathrm{s}$.

The benefits of structured modulator software are well known. This is especially true for large complex systems, such as the FOC motor control, with many sub-blocks. It reduces the developing time, and could be reused in the future project. Therefore, a typical 
incremental build process is used in this research paper.

$* * * * * * * * * * * * * * * * * * * * * * * * * * * * * * * * * * * * * * * * * * * * * * * * * * * * * * * * * * * * * * * * * *$

Phase commissioning options

$* * * * * * * * * * * * * * * * * * * * * * * * * * * * * * * * * * * * * * * * * * * * * * * * * * * * * * * * * * * * * * * * * * *$

phase_commissioning1 .set 1 ; hardware checkup

phase_commissioning2 .set $\quad 0 \quad$; open loop start up

phase_commissioning 3 .set $\quad 0 \quad$; current measurement chain checking

phase_commissioning 4 .set $0 \quad$; current regulation implementation

phase_commissioning5 .set $0 \quad$; Kalman filter estimation

phase_commissioning6 .set $\quad 0 \quad$; closed current and speed loop

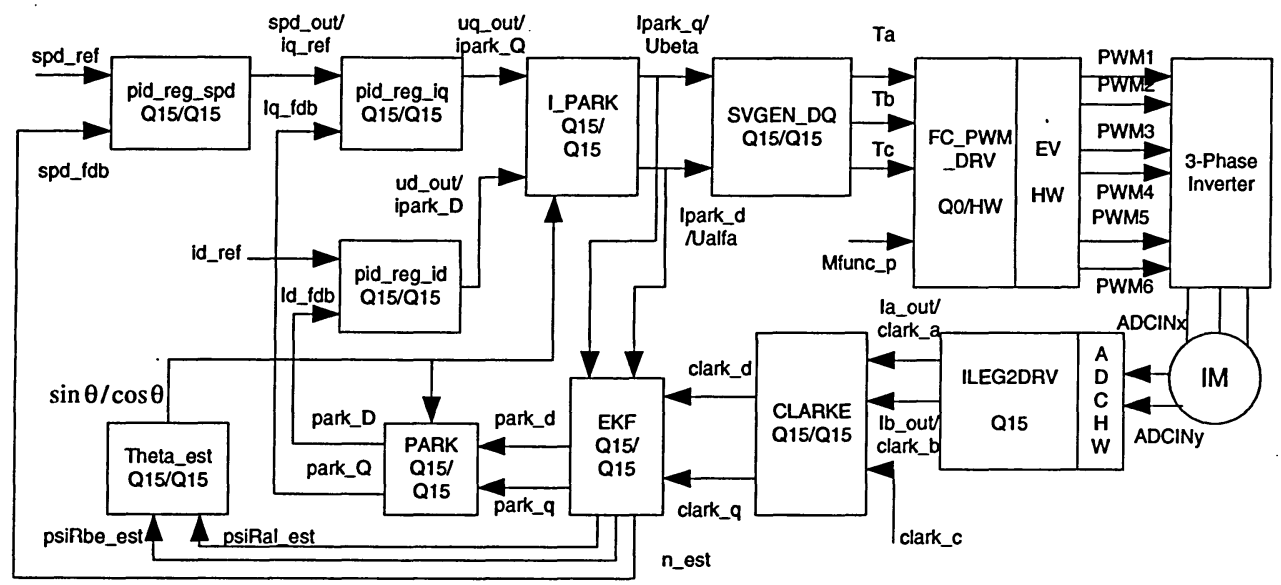

Figure 17: EKF Software Structure for an Induction Motor

The Figure 17 shows the block diagram of the software developed in this research paper. The system implemented here uses the following assembly software modules, which meet the requirements of reusability, compatibility, predictability and expandability. Appendix D gives all of the software modules used in this research paper.

CLARKE: $\quad$ Clarke transformation module converts the three-phase stator currents into two-phase quadrature currents.

EKF: $\quad$ Extended Kalman filter computes the rotor flux and speed.

DATA_LOG: Store the real-time values of two selected variables for graphical display.

I_PARK: Inverse Park transformation converts rotating reference frame into stationary frame. 
ILEG2DRV-ADC: Convert two current inputs $i_{a}, i_{b}$ into digital representations.

PARK: $\quad$ Park transformation converts two-phase $\alpha-\beta$ stationary frame into $\mathrm{d}-\mathrm{q}$ rotating reference frame.

$P I D \_R E G \_I D / I Q$ : Control module for regulatinge currents $i_{d}, i_{q}$.

PID_REG_SPD: Control module for speed control loop.

FC_PWM_DRV: Generate PWM outputs.

THETA_EST: Calculate the sine value and cosine value of rotor flux angle $(\sin \theta / \cos \theta)$ for Park transformation and inverse Park transformation.

RAMP_GEN: Generate $60 \mathrm{~Hz}$ saw-tooth waveform to emulate the rotor flux angle in debugging.

SINTB360: Look-up table for sine value from $0^{\circ}$ to $360^{\circ}$.

$S Q R T_{-} T A B: \quad$ Square-root table from 0 to 1.22 .

SVGEN_DQ: Use space vector PWM technique to calculate duty ratios for PWM1-PWM6.

SYS_INIT: Initialize the software.

\subsection{Debugging of Software and Hardware}

After the hardware work and software programming have been completed, the software needs to be carefully debugged. The Incremental Build Process plays a key role in debugging, which decomposes the whole control task into several small tasks. There are totally six phases in debugging of the control system:

Phase 1: Confirm operation of hardware connection and the code framework.

Phase 2: Check the forward control path and PWM driver.

Phase 3: Use the $60 \mathrm{~Hz}$ open-loop start to check the current sensing and feedback path.

Phase 4: Check the correct operation of the current regulators.

Phase 5: Examine the correct operation of extended Kalman filter estimation and insert it in the control system.

Phase 6: Implement close loop control of current and speed. 
For simplicity, Phase 2 is explained as an example. The other phase debugging procedure can be found in the software of main program foc2.asm (Appendix D).

\section{a) Goal of the Phase 2}

The objective of this phase is to check the PWM driver and PWM outputs with a simple RC filter. Following this verification, the induction motor will be connected to the power inverter board and started to run smoothly

\section{b) Building blocks interconnection}

Figure 18 shows all software modules to be used in Phase 2. The output of the inverse Park module "I_PARK" is used as the stator voltage reference. The amplitude and phase of this voltage vector are translated into commutation durations by the SVGEN_DQ module, which uses the space vector PWM technique. The FC_PWM_DRV module uses the timing information to generate the PWM outputs to control inverter for driving the induction motor. The DATA_LOG module provides a dual memory buffer to display two graphical waveforms in real time, which could be used to show any interested variable such as $T a, T b, T c$ or PWM outputs. The RAMP_GEN module is used to generate a $60-\mathrm{Hz}$ saw-tooth waveform to emulate the rotor flux angle in debugging.

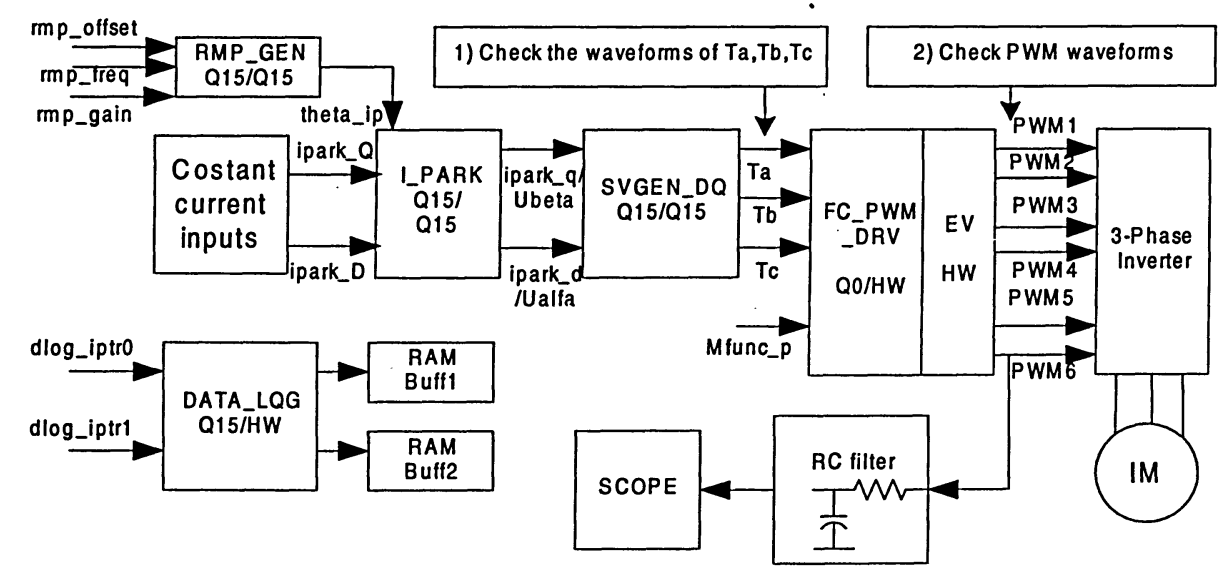

Figure 18: Overview of Phase 2 Software Flow

c) Hardware and software debugging in Phase 2 
1. Select the build option phase_commissioning 2 set 1 (the others stay to zero) in the main program foc2.asm, then build the project and load the program foc2.out and the corresponding workspace (see Figure 19).

2. Connect a RC filter to one PWM output.

3. Verify that the filtered waveform.

4. The values of resistor and capacitor in the RC low-pass filter can be changed to have different cutoff frequencies, which must be well below the PWM carrier frequency.

5. Connect the PWM outputs to the power inverter.

6. Increase the input voltage gradually until the motor starts running.

7. Change the value of $r m p$ freq (one of inputs in RAMP_GEN module) in a real-time watch window to vary the motor speed.

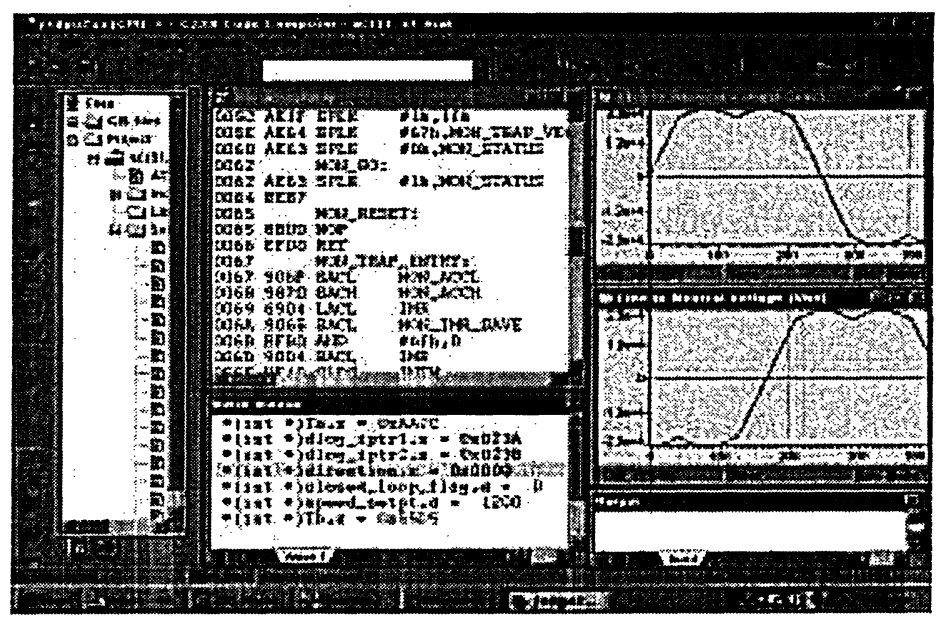

Figure 19: Overview of Phase 2 Workspace

It should be pointed out that the real time watch window is very helpful in the debugging period, since it can be used to watch each internal variable defined in the software and can be updated continuously. 


\section{Chapter 5 \\ Experimental Verification of \\ Field Oriented Motor Control using Kalman Filter}

This chapter presents the experimental verification of the field oriented control of induction motor using Kalman filter. Typical measurements are given to demonstrate the efficiency of the novel control designed in this research paper.

The following outlines the sections in this chapter.

Section 5.1 presents all the waveforms of the internal states in the close-loop speed control to demonstrate the efficiency of the control process designed in this research paper.

Section 5.2 demonstrates the dynamic response of the speed sensorless control scheme, and results of the speed-step response are given.

Section 5.3 shows the quality of the speed sensorless control, and results of the relative speed errors are given.

\subsection{Internal States of Closed-Loop Control}

This section presents all the waveforms of the internal states in the close-loop speed control to demonstrate the efficiency of the control process designed in this research paper. The control scheme was shown in Figure 17 (Chapter 4). In the following demonstration, the reference speed spd_ref was set to $1758 \mathrm{rpm}$

1) Phase currents $\left(i_{a}, i_{b}\right)$ are measured by current transducers, then the outputs of the transducers are converted into digital signals (Ia_out, Ib_out) by two analog-to-digital (ADC) channels in the DSP analog interface. Figure 20 shows the phase currents. 


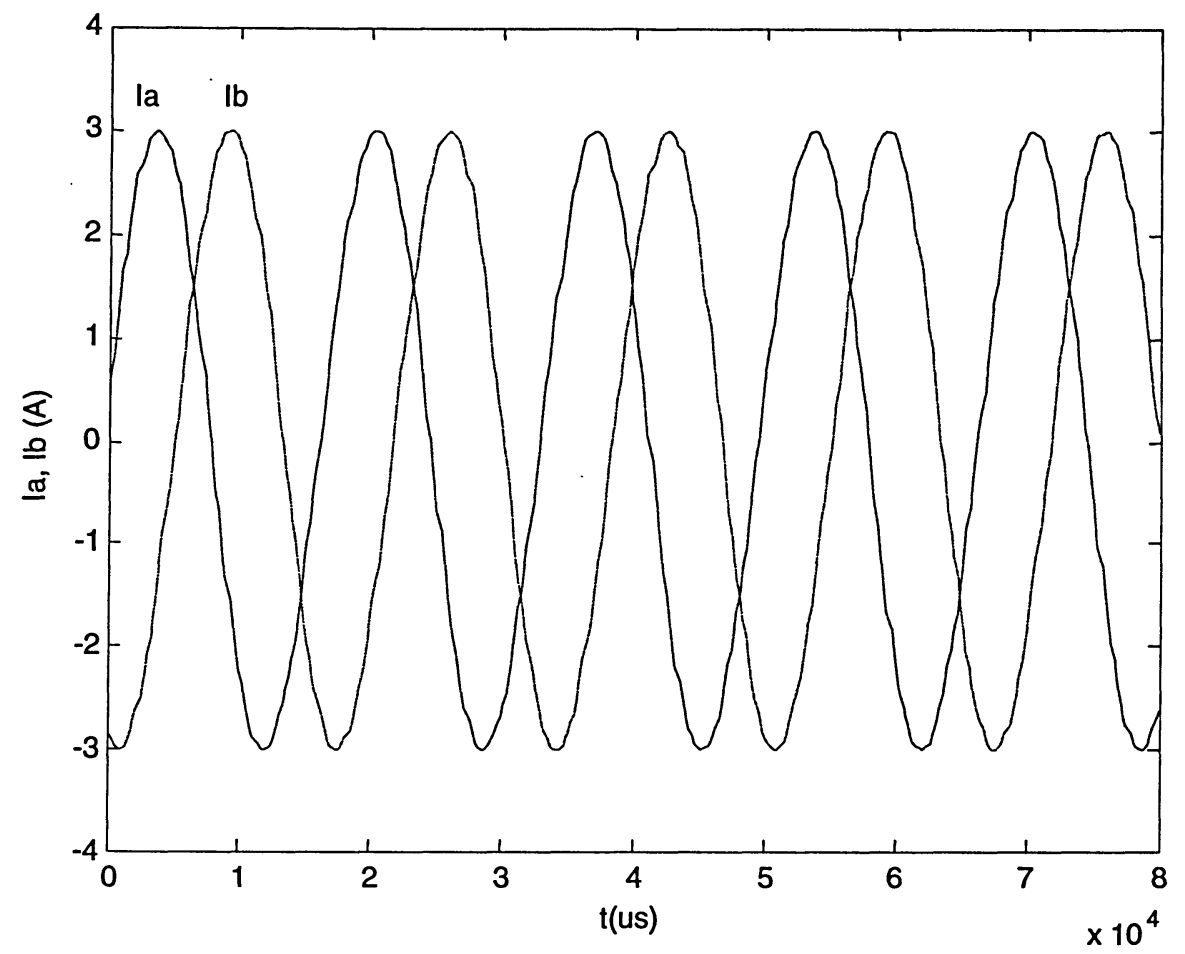

Figure 20: $\quad i_{a}, i_{b}$ Currents from ADC

2) The digital signals of the two phase currents are transformed into $\alpha-\beta$ currents (clark_d, clark_q) using Clarke transformation. Figure 21 shows the $\alpha-\beta$ currents.

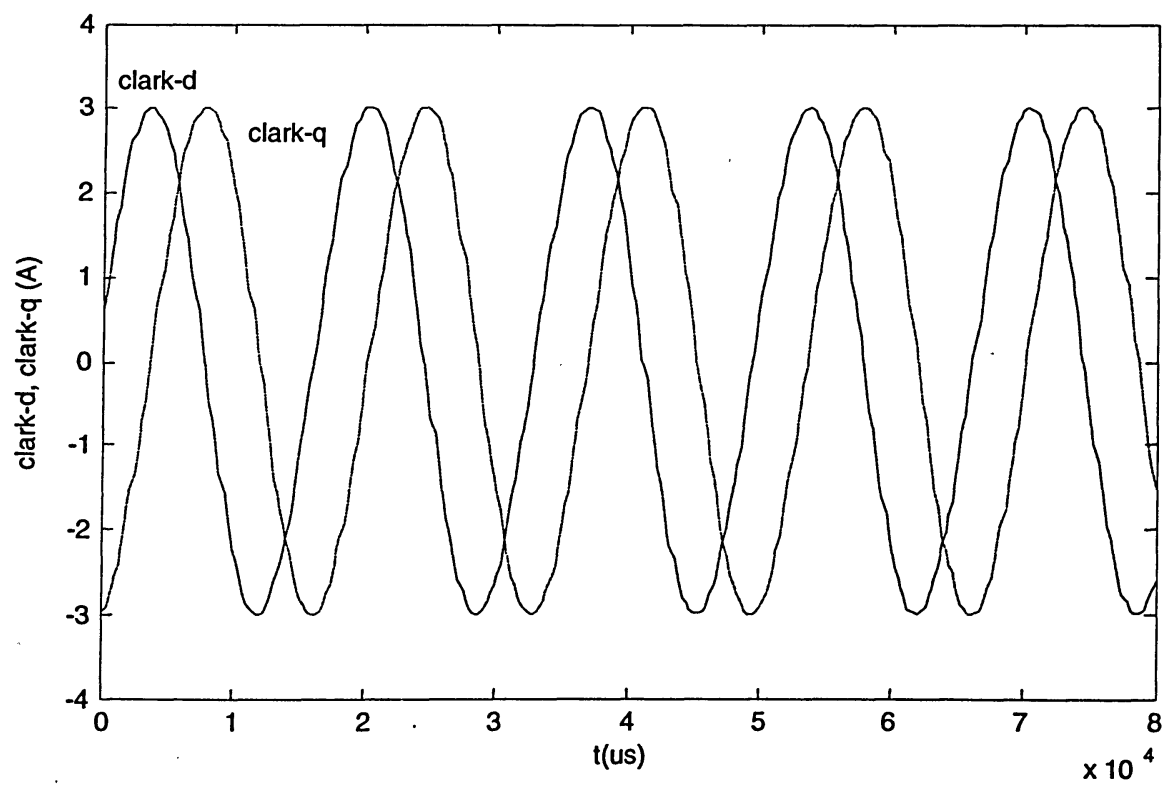

Figure 21: Currents from Clarke Transformation 
3) Kalman filter module has four inputs (two currents clark_d, clark_q and two voltages Ubeta,Ualfa) and five estimate outputs (two currents iSal_est, iSbe_est, two rotor fluxes psiRal_est, psiRbe_est and one speed estimation $\left.n \_e s t\right)$. Figure 22 and Figure 23 show the estimated outputs from the Kalman filter module.

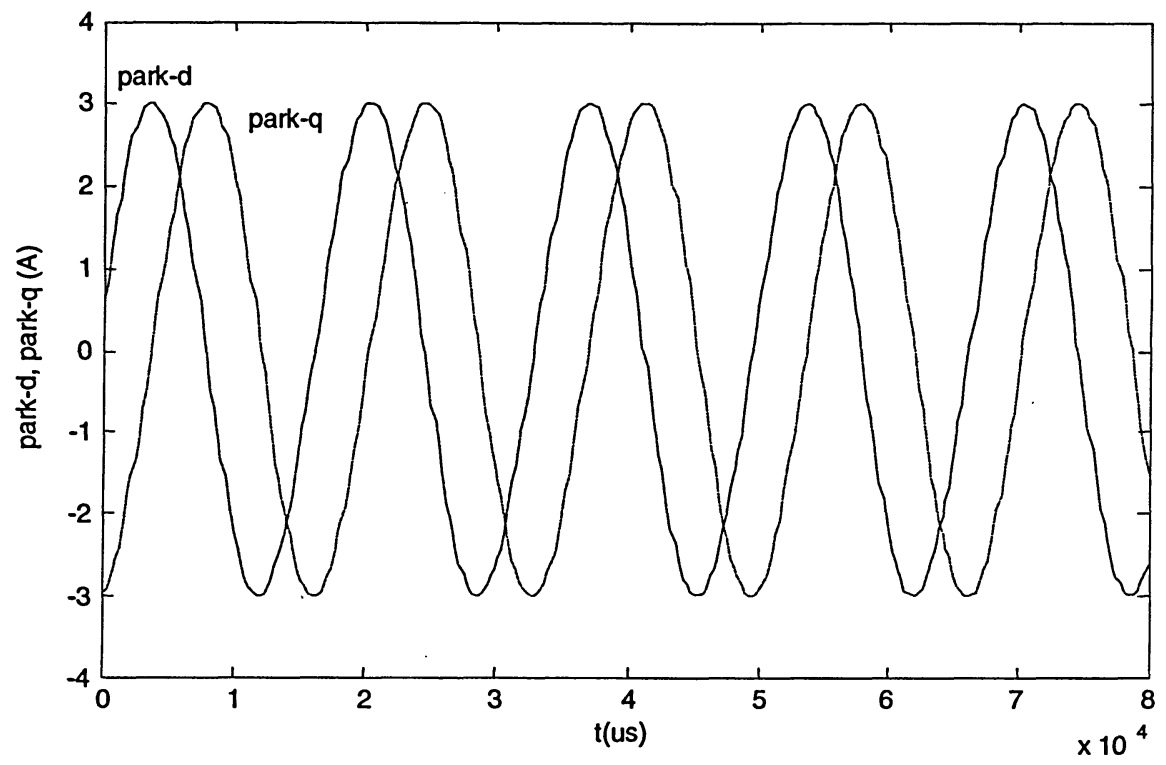

Figure 22: Current Estimated by Kalman Filter

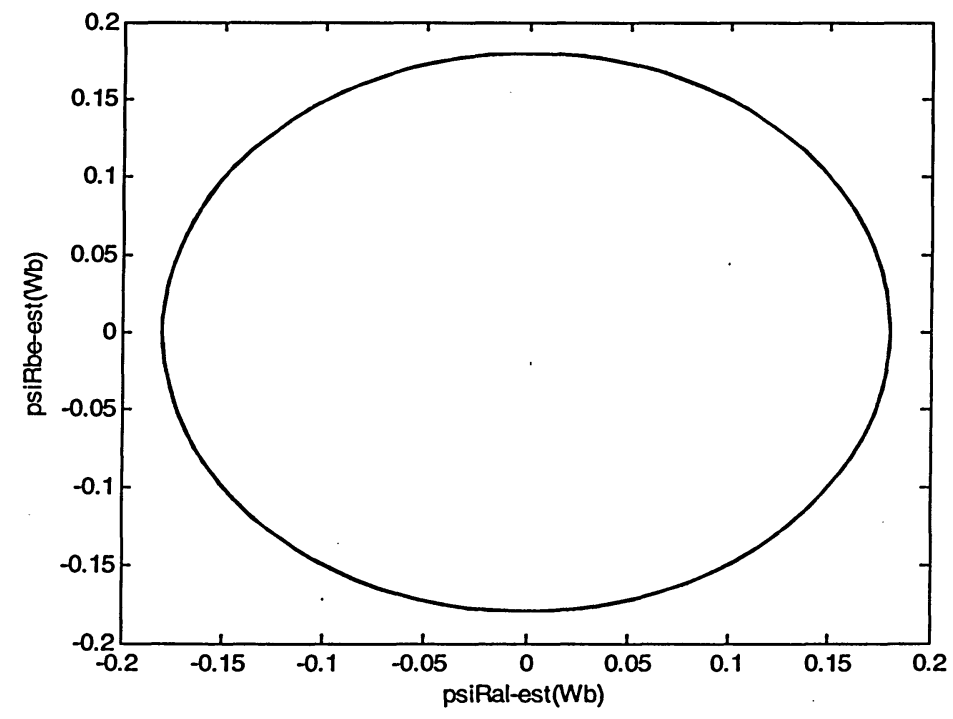

Figure 23: Flux Circle Estimated by Kalman Filter

4) $\alpha-\beta$ currents (park_d, park_q) computed by Kalman filter module are transformed into two quadrature currents (park_ $D$, park_Q ) with reference to the rotor flux using extended Park transformation. Figure 24 shows the quadrature currents. 


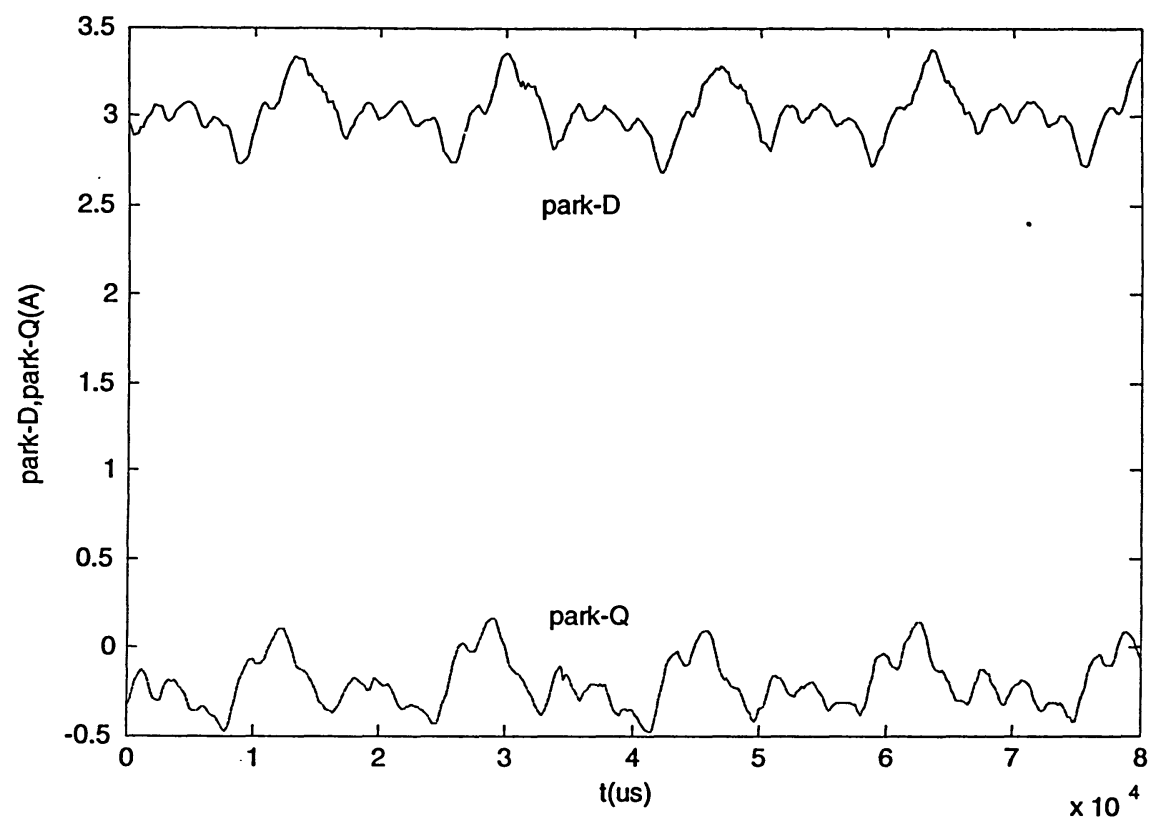

Figure 24: Currents from Park Transformation

5) Speed regulator has two inputs. One is reference speed (spd_ref), and the other is speed feedback $\left(s p d \_d b\right)$ estimated by Kalman filter. The output of the regulator is used as the reference $i_{q}$ current (iq_ref). Figure 25 shows the speed control.

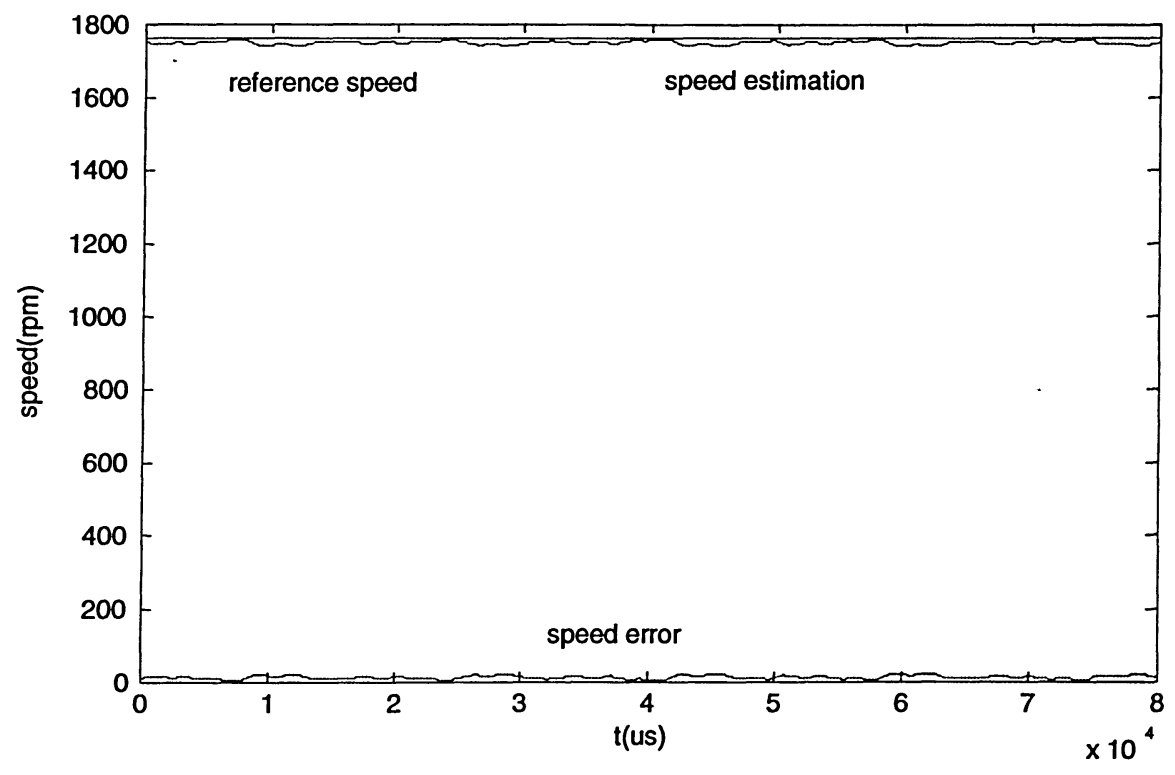

Figure 25: Speed Control 
6) There are two regulators for $i_{d}$ and $i_{q}$. The $i_{d}$ regulator has two inputs: one is reference $i_{d}\left(i d \_r e f\right)$, which is dependent on the flux requirement; the other is feedback $i_{d}$ (id_fdb), which is obtained from the Park transformation. Similarly, the $i_{q}$ regulator has two inputs: one is reference $i_{q}\left(i q_{-} r e f\right)$, which is dependent on the speed requirement; the other is feedback $i_{q}(i q f d b)$, which is obtained from the Park transformation. Figure 26 shows the torque control and Figure 27 shows the flux control. Figure 28 shows the outputs from the two regulators.

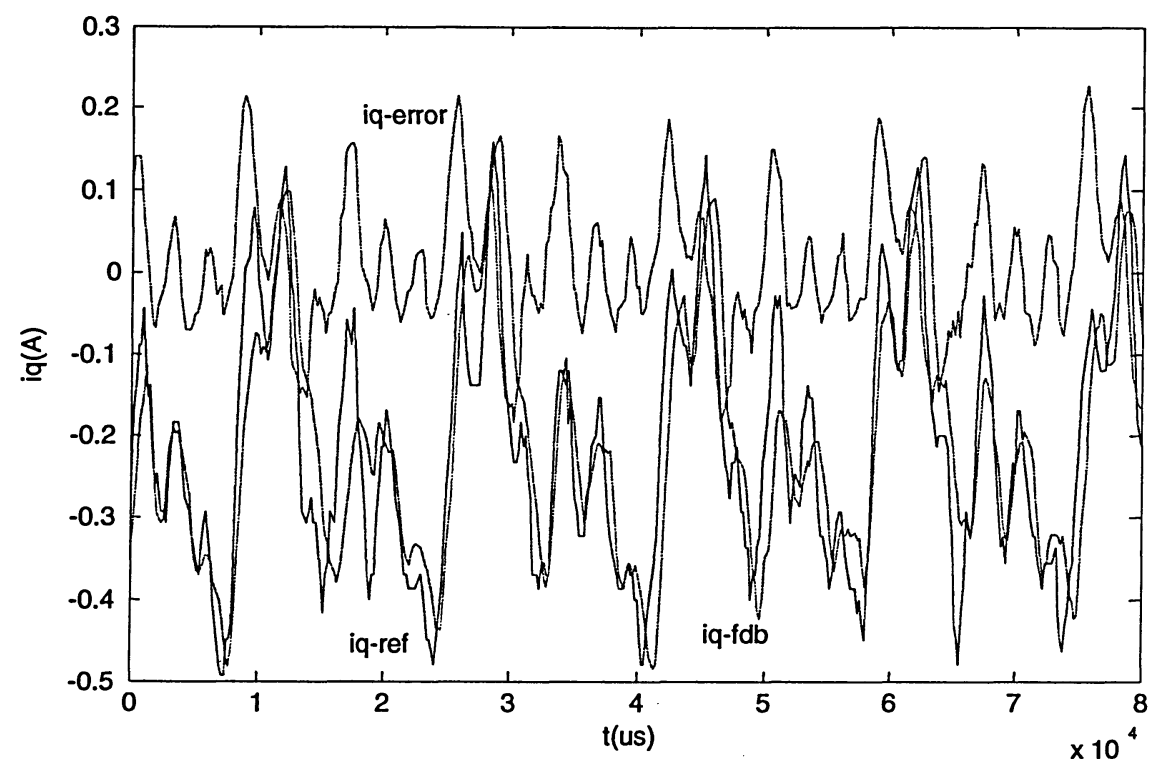

Figure 26: $i_{q}$ (torque component) Control

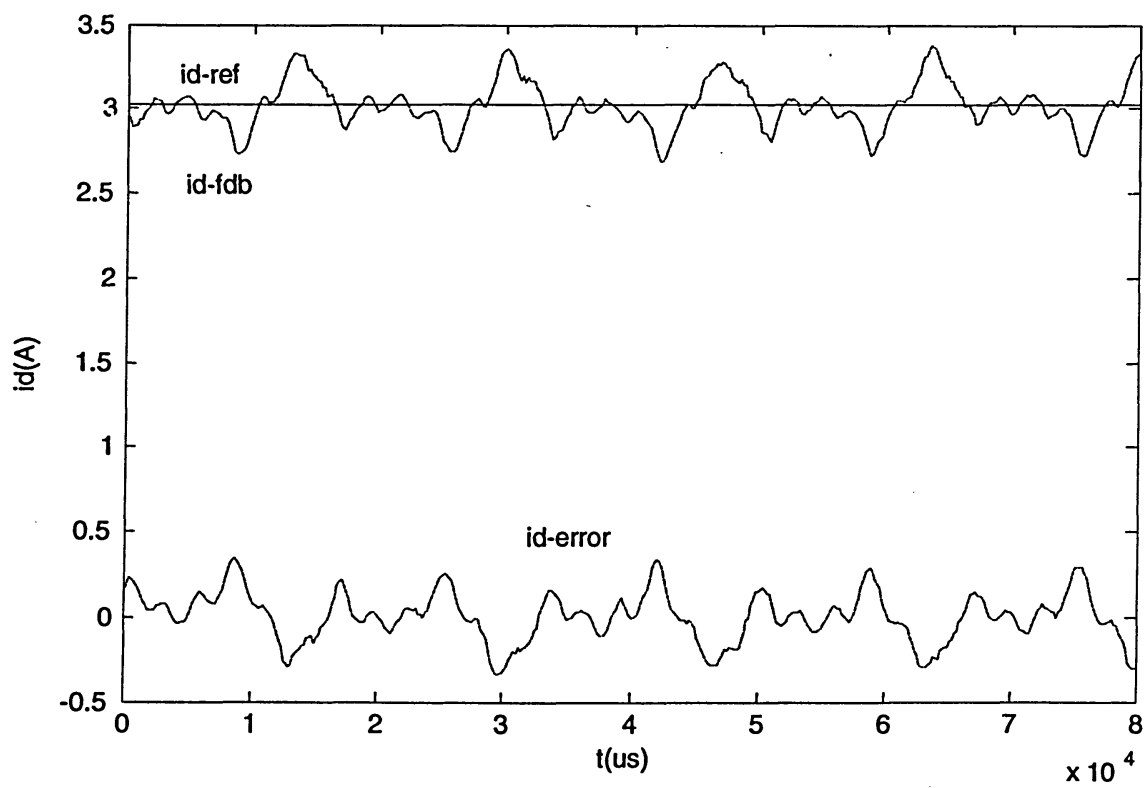

Figure 27: $i_{d}$ (flux component) Control 


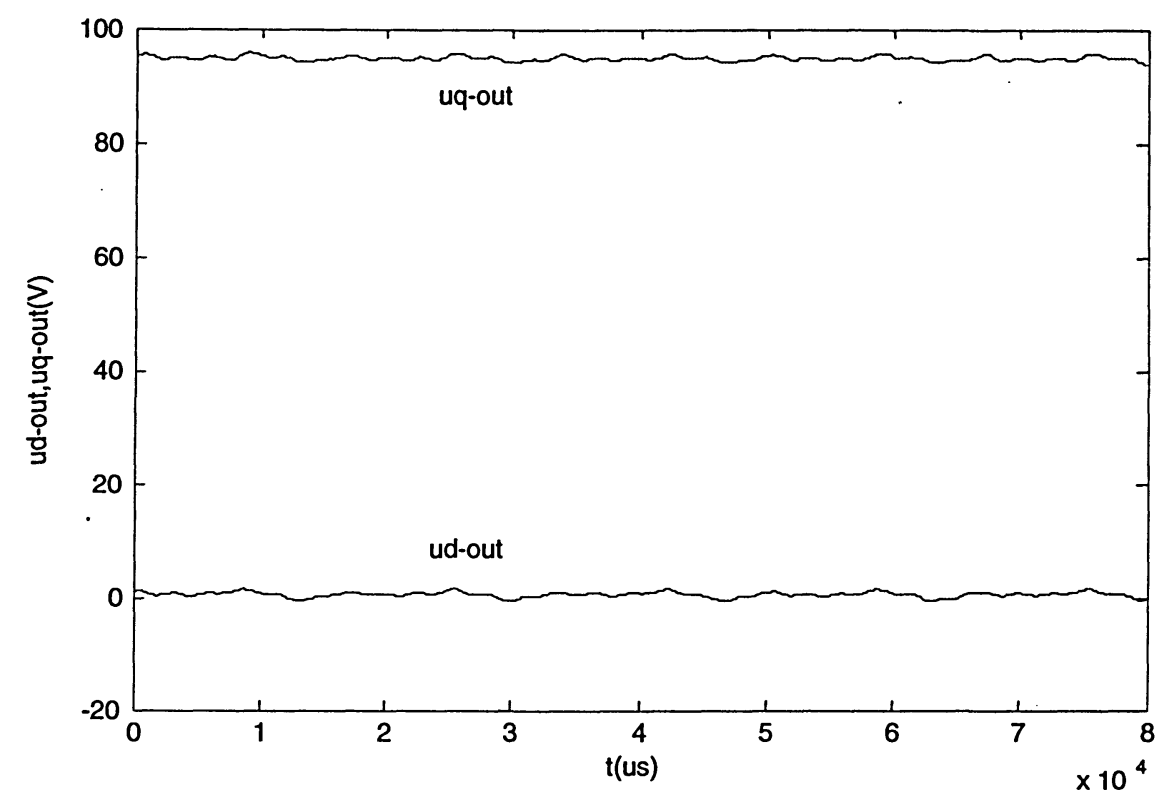

Figure 28: Voltage Outputs from $i_{d}, i_{q}$ Regulators

7) Inverse Park transformation is used to convert the outputs of the two current regulators (ud_out, uq_out) into $\alpha-\beta$ voltages (Ualfa, Ubeta). Figure 29 shows the $\alpha-\beta$ voltages.

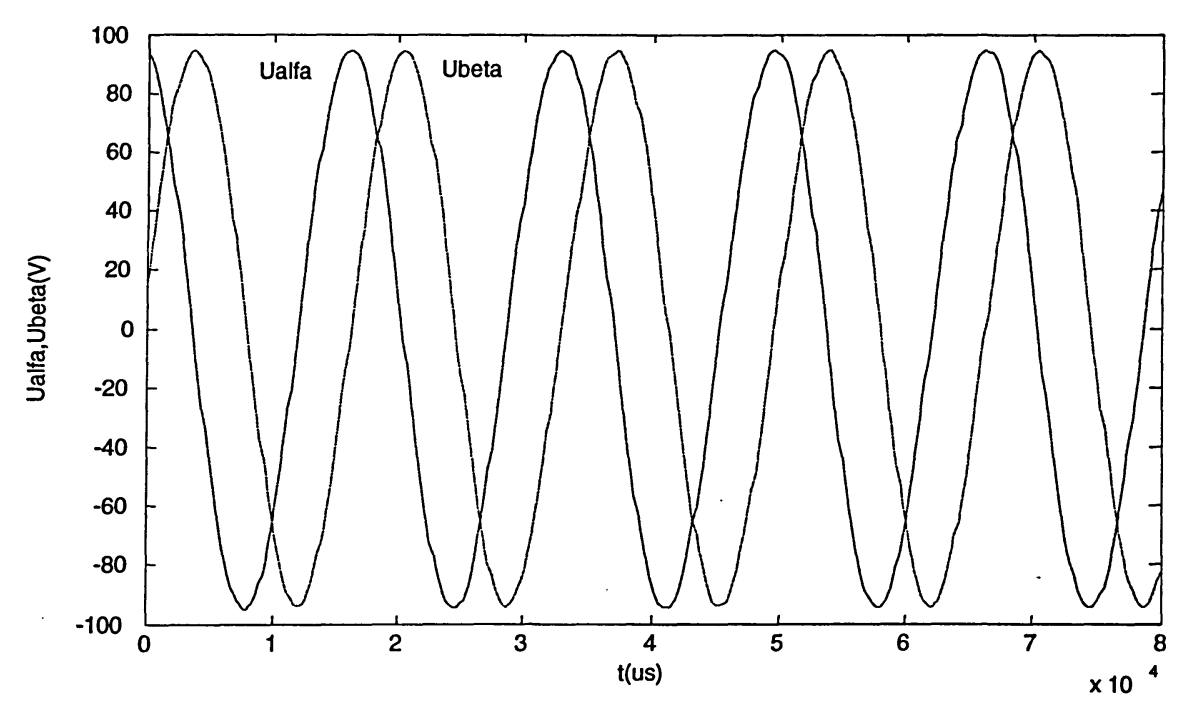

Figure 29: Ualfa, Ubeta from Inverse Park Transformation

8) Flux angle estimation (theta_est) is used to calculate the sine and cosine values of flux angle, which are crucial to Park and inverse-Park transformations, based on the estimated flux inputs from Kalman filter.

9) Given the required $\alpha-\beta$ voltages (Ualfa, Ubeta), space vector PWM module is used to generate corresponding $O N$ durations: $T a, T b$ and $T c$ in a sample period $T$. Figure 30 
shows the durations.

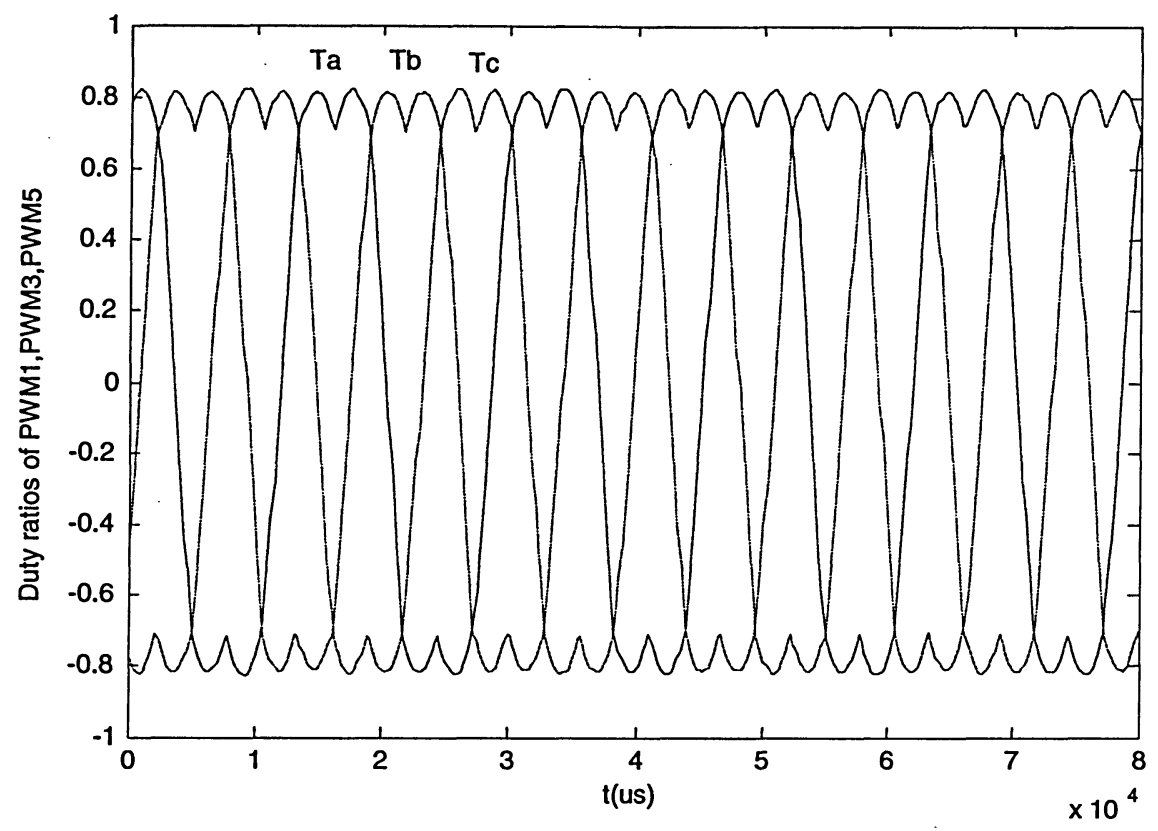

Figure 30: Duty Ratios of PWM1, PWM3 and PWM5 in Carrier Period

10) Three full compare units in the DSP are used to produce the PWM1-PWM6 based on the $T a, T b, T c$ and sample period $T$.

11) The PWMs from the DSP are fed to the voltage source inverter (VSI). The outputs of the VSI are three-phase voltages used to control the induction motor. Figure 31 shows the PWM outputs from the IGBT module and Figure 32 shows the line-to-line voltages fed to the induction motor.

From above figures, the following results can be obtained.

1) $i_{a}$ leads $i_{b} 120$ degrees.

2) After Clark transformation, clark_d leads clark_q by 90 degrees.

3) Currents (park_d, park_q), estimated by Kalman filter, are almost the same as the clark_d and clark_q.

4) After Park transformation, $i_{d}, i_{q}$ currents (Park_D,Park_Q) are close to dc currents.

5) The performances of the speed control, $i_{d}$ regulation and $i_{q}$ regulation are very good.

6) Ualfa leads Ubeta by 90 degrees.

7) Flux is constant in the control process.

8) $T a$ leads $T b 120$ degrees, $T b$ leads $T c 120$ degrees.

9) Phase voltages are 120 degrees shift from each other. 


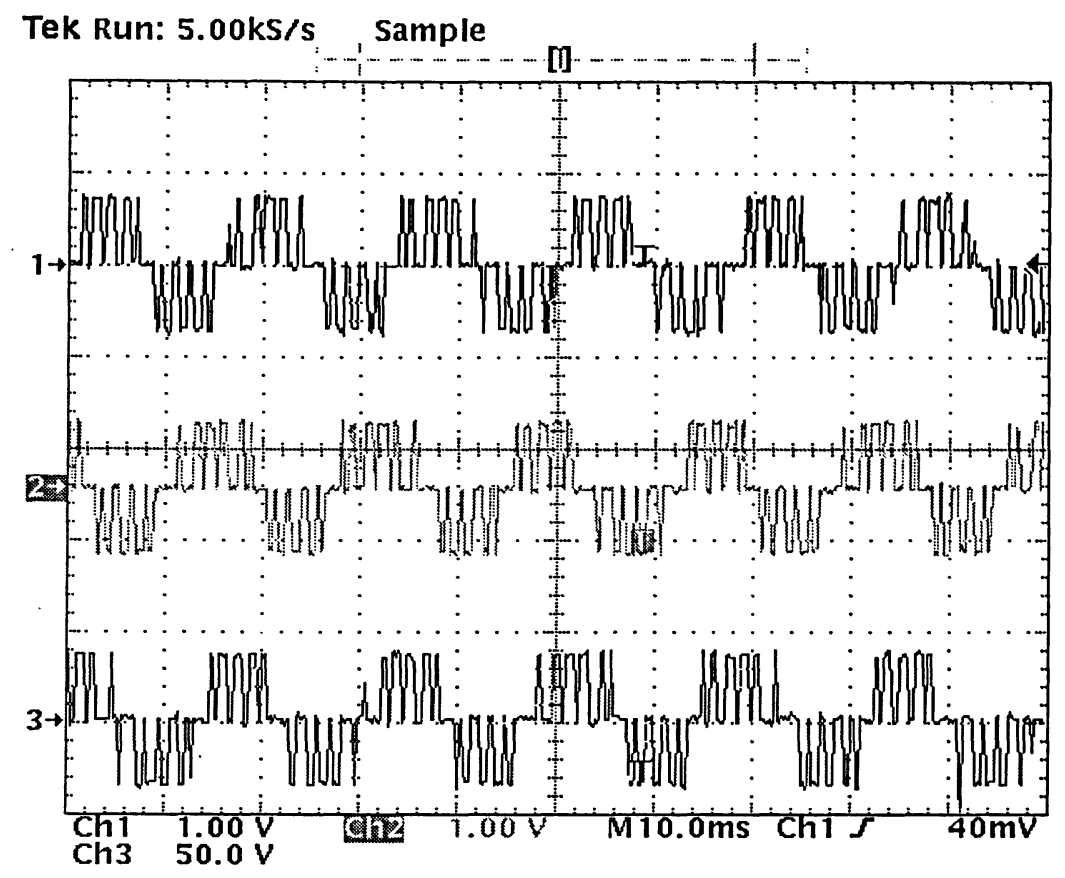

Figure 31: PWM Outputs of IGBT Power Module

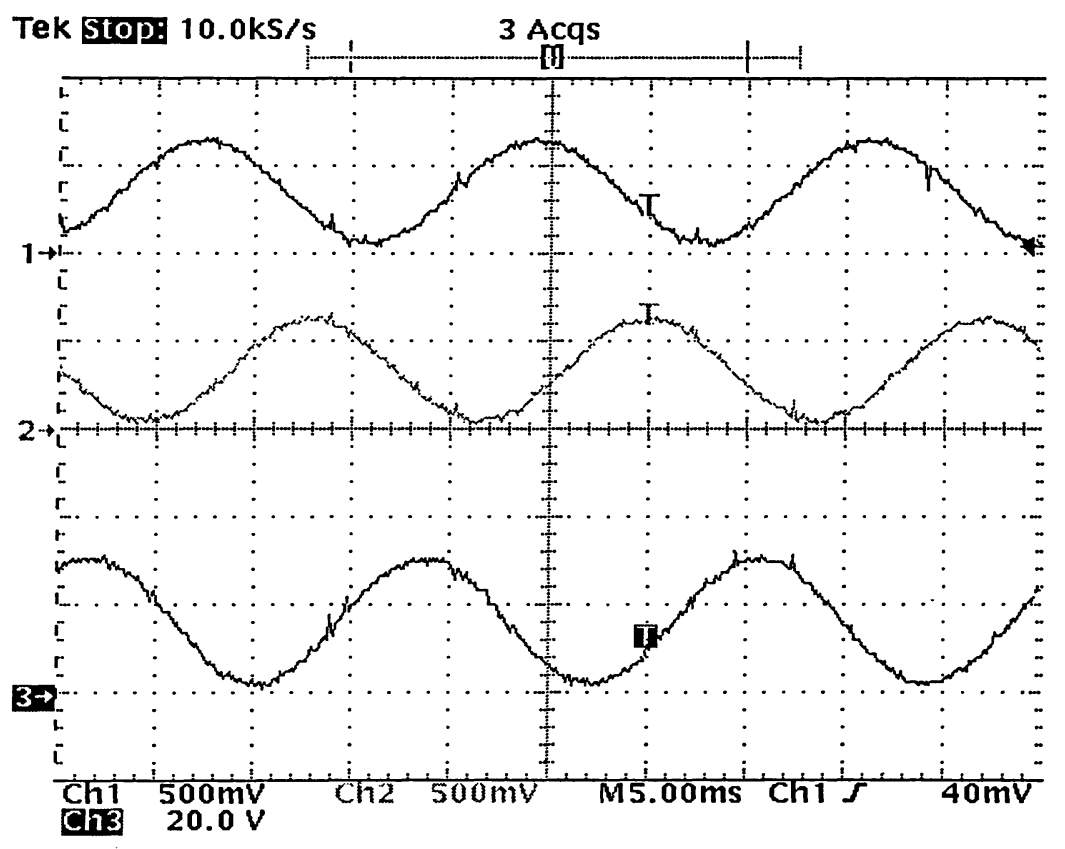

Figure 32: Line to Line Voltages of the Induction Motor 


\subsection{Dynamic Response}

This section demonstrates the dynamic response of the speed sensorless control scheme. Figure 33 shows speed-step response from 500rpm to 1500rpm. The scope Channel \#1 is the speed reference, Channel \#2 is the measured speed, Channel \#3 is the flux component $i_{d}$, and Channel \#4 is the torque component $i_{q}$.

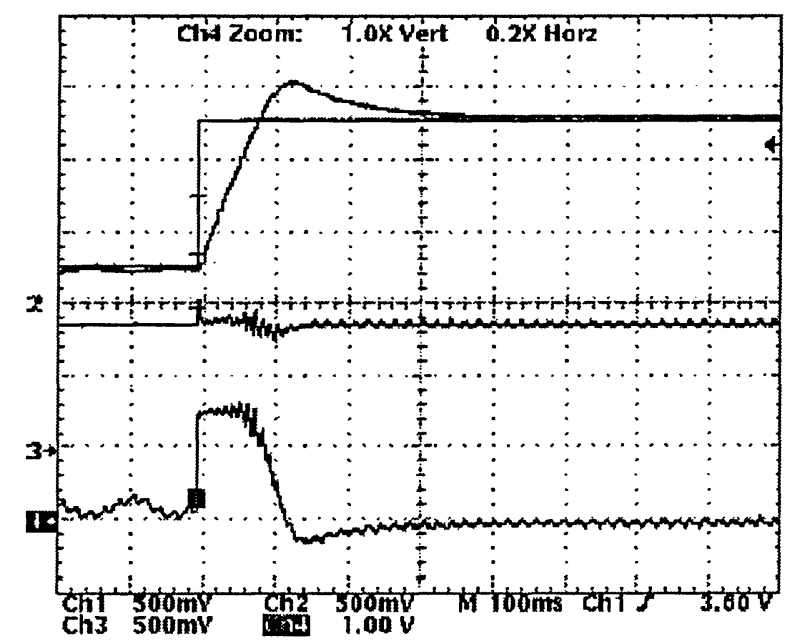

(a) without load

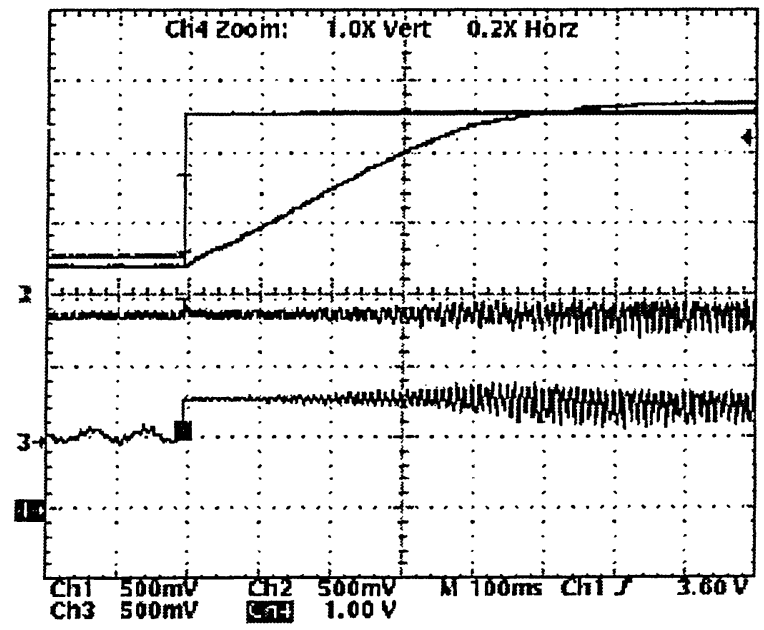

(b) with load

\section{Figure 33: Speed Step Response (from 500rpm to 1500rpm)}

The Figure 33a shows the speed control without load. Figure 33b shows the speed control with load (another EPACT motor is used as a load). The response time varies between 
$500 \mathrm{~ms}$ and $800 \mathrm{~ms}$. Figure 34 shows the stator currents starting from 0 to $1000 \mathrm{rpm}$.

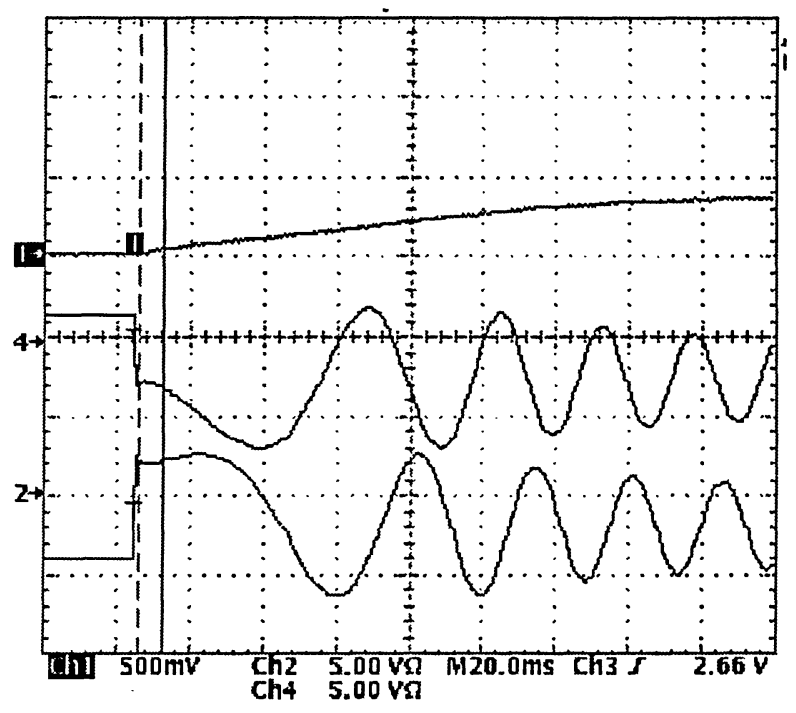

Figure 34: Speed Transient from 0 to 1000rpm

Ch1: n: mechanical speed; Ch2: Ib stator phase current; Ch4: Ia stator phase current

The following scope picture shows a speed reversion test from -1000rpm to 1000rpm with load.

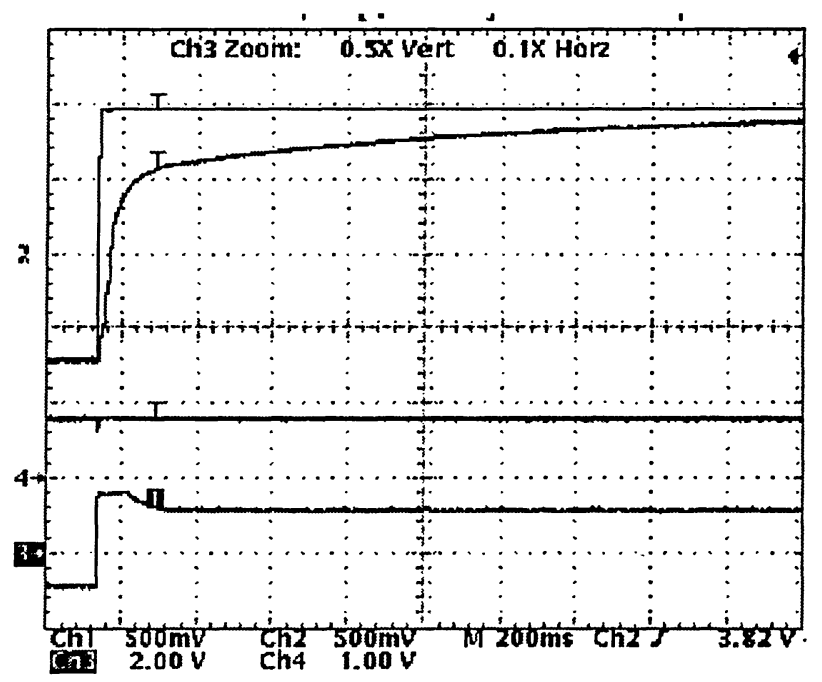

Figure 35: Speed Reversion (from -1000rpm to 1000rpm) 


\subsection{Speed Control Accuracy}

For testing the quality of the speed sensorless control performance, the relative error in speed is defined as follows:

$$
e(\%)=\frac{n-n_{r e f}}{n_{r e f}} 100 \%
$$

Table 1 shows the relative speed error at different speeds. The measurements run from 500 rpm up to $1800 \mathrm{rpm}$ (the nominal speed of the induction motor used in the test).

\section{TABLE 1}

\section{Steady State Performance of Speed Sensorless Control}

\begin{tabular}{|l|l|l|l|}
\hline $\begin{array}{l}\text { Reference speed } \\
(\mathrm{rpm})\end{array}$ & $\begin{array}{l}\text { Measured speed } \\
(\mathrm{rpm})\end{array}$ & $\begin{array}{l}\text { Estimated speed } \\
(\mathrm{rpm})\end{array}$ & $\begin{array}{l}\text { Speed control error } \\
(100 \%)\end{array}$ \\
\hline 549.3 & 550.5 & 550.8 & $0.22 \%$ \\
\hline 824.0 & 818.9 & 819.6 & $-0.62 \%$ \\
\hline 1098.6 & 1095.0 & 1096.5 & $-0.33 \%$ \\
\hline 1373.3 & 1376.0 & 1377.6 & $0.20 \%$ \\
\hline 1648.0 & 1650.0 & 1651.5 & $0.12 \%$ \\
\hline 1702.9 & 1702.0 & 1702.5 & $-0.05 \%$ \\
\hline 1757.9 & 1758.0 & 1759.5 & $0.005 \%$ \\
\hline 1785.3. & 1789.0 & 1790.7 & $0.21 \%$ \\
\hline
\end{tabular}




\section{Chapter 6}

\section{Conclusions}

This research paper has presented a novel method for the speed control of induction motors without using a speed sensor. The rotor speed is accurately computed using the Kalman filter software module designed in this research paper. The computation, which has been implemented in the DSP TMS320LF2407, simply uses the two current measurements at the motor terminal. This replaces a speed sensor and eliminates the difficulty of the sensor installation in many applications.

This research paper has presented an advanced field oriented control of induction motors based on a specific d-q coordinate model, with the d-coordinate chosen to be in line with the rotor flux and the q-coordinate chosen to be $90^{\circ}$ lagging. The position of the rotor flux is accurately computed using the Kalman filter module. This eliminates the position sensors required to monitor the flux. This research paper has shown that as a result of the specific $\mathrm{d}-\mathrm{q}$ transformation, the motor torque is proportional to the product of the rotor flux and the q-coordinate stator current. This significantly simplifies the induction motor control, such that the rotor flux is controlled simply by regulating the flux-related d-coordinate stator current and the motor torque is controlled just by regulating the q-coordinate stator current.

This research paper has presented the Kalman filter which is an optimal control observer and its recursive algorithm and formulations which are specifically designed for the induction motor control. The Kalman filter provides the minimum variance state estimation and tolerates system modeling and measurement errors, which are considered as noise processes in the state estimation. The filter processes all available measurements regardless of their precision, and provides a quick and optimal estimate of the variables of interest, as well as achieves a fast convergence.

This research paper has presented the implementation of the field oriented control of induction motors using Kalman filter. The hardware requirements and all software modules are detailed. The experimental verification of the control method designed in this research paper has been provided. Typical measurements are given to demonstrate the efficiency of the novel control designed in this research paper.

\section{Key Contributions of this Research Paper}

The following lists the key contributions in this research paper.

1. Full investigation of Kalman filter for the control of induction motors. 
This research paper has provided a full investigation of the practical application of Kalman filter for the control of induction motors. There has been very limited information about the use of Kalman filter for induction motor drives, possibly because of the difficulty of understanding the theory of the Kalman filter. This research paper research has carried out a detailed study of the control theory of the Kalman filter and its suitability for the control of induction motors. This research has provided a concrete finding that Kalman filter is well suitable for induction motor control.

2. Detailed formulations of the specific field oriented control with Kalman filter for real-time control of induction motors.

This research paper has developed all formulations required for the specific field oriented control of induction motors using Kalman filter. In particular, a recursive algorithm of the Kalman filter is given in the research paper. The algorithm and its corresponding formulations has provided an accurate computation of the rotor speed, the flux linkage, and the flux angle which are the essential components for the specific field oriented control of induction motors.

3. DSP implementation of the specific field oriented induction motor control.

This research paper has developed the software required for the implementation of the specific field oriented motor control in DSP TMS320LF2407. The software is developed in modules which can be easily modified for different induction motor drive applications.

4. Real-time verification of the specific field oriented control using Kalman field.

This research paper has built a prototype of the induction motor drive to verify the specific field oriented control using Kalman filter. Typical measurements are given to demonstrate the efficiency of the novel control designed in this research paper.

\section{Future Work in Field Oriented Control using Kalman Filter}

This research paper has provided a concrete proof that Kalman filter can be used to accurately compute the motor speed and flux which are crucial for the specific field oriented control of induction motors. The efficiency of the novel control designed in this research paper has been demonstrated. Key future researches in the area of the induction motor control are proposed in the following.

1. Tune the $\mathrm{Q}$ and $\mathrm{R}$ matrices in the Kalman filter to get better performance. 
$\mathrm{Q}$ and $\mathrm{R}$ are the covariance matrices of the state and measurement noises in the Kalman filter formulation. To date, there has been no exceptional good method to determine the matrices of $Q$ and $R$.

2. Find lower order of the state equation for the Kalman filter computation.

If a lower order state equation, instead of the 5-order equation presented in this research paper, can be found and can represent accurately the rotor speed and flux, the computation can be significantly reduced. The reduction of computation time is very important for the real-time control of induction motors.

3. Compare the motor drive with or without a speed sensor.

Usually the sensorless control has good performance in the normal motor speed. However, the performance may deteriorate in very low motor speed. In fact, low speed sensorless control for induction motors is a hot topic in recent research on sensorless control. 


\section{Bibliography}

[1] R. Di Gabriele, F. Parasiliti, M. Tursini, "Digital Field Oriented Control for induction motors: implementation and experimental results", Universities Power Engineering Conference (UPEC'97)

[2] Texas Instruments, "Implementation of a Speed Field Orientated Control of Three phase AC Induction Motor using TMS320F240", Literature number: BPRA076, January 1998

[3] Texas Instruments, "Field Orientated Control of Three phase AC-motors", Literature number: BPRA073, December 1997

[4] R. Di Gabriele, F. Parasiliti, M. Tursini, "Digital Field Oriented Control for induction motors: implementation and experimental results", Universities Power Engineering Conference (UPEC'97)

[5] L. Loron, G. H. L. Peterson, "Application of an Extended Kalman Filter to the estimation of the parameters of a solid rotor asynchronous drive with two degrees of freedom", EPE 1991, Firenze

[6] Texas Instruments, "Sensorless Control with Kalman Filter on TMS320 Fixed-Point DSP", Literature number: BPRA057, July 1997

[7] A. Germano, F. Parasiliti, M.Tursini, "Sensorless Speed Control of a PM Synchronous Motor drive by Kalman Filter", International Conference on Electrical Machine (ICEM '94)

[8] C. Manes, F. Parasiliti, M.Tursini, "A Comparative Study of Rotor Flux Estimation in Induction Motors with a Nonlinear Observer and the Extended Kalman Filter", IEEE International Conference on Power Electronics, Control and Instrumentation (IECON '94)

[9] Werner Leonard, "Control of Electrical Drives", 2nd Completely Revised and Enlarged Edition, Springer, ISBN 3-540-59380-2

[10] D. W. Novotny and T. A. Lipo, "Vector Control and Dynamics of AC Drives", Oxford Science Publications, ISBN 0-19-856439-2

[11] K. Astrom, B. Wittenmark, Adaptive Control, Addison Wesley, 1995.

[12] C. Phillips, H. Nagle, Digital Control Systems Analysis and Design, Prentice Hall, 1995. 
[13] Y. Dote, Servo Motor and Motion Control Using Digital Signal Processors, Prentice Hall, 1990.

[14] J. Tang, R. Chassaing, "PID Controller Using the TMS320C31 DSK for Real-Time DC Motor Control," Proc. of the 1999 Texas Instruments DSPS Fest, Houston, Texas, August, 1999.

[15] B. Widrow, S. Stearns, Adaptive Signal Processing, Prentice Hall, 1985

[16] R. Krishnan, Electrical Motor Drives: Modeling, Analysis, and Control, Prentice Hall, 2001. 


\section{Appendix A}

\section{Summary of Notations}

\begin{tabular}{|l|l|}
\hline \multicolumn{1}{|c|}{ Meaning } & \multicolumn{1}{c|}{ Notation } \\
\hline Electrical and Mechanical Torque & $\mathrm{T}_{\mathrm{e}}, \mathrm{T}_{\mathrm{L}}$ \\
Flux & $\Psi$ \\
Flux Angle & $\theta$ \\
Flux Speed & $\omega_{m R}$ \\
Inertia & $J$ \\
Leakage Factor & \\
Magnetic Pole Number & \\
Magnetizing Current & $i_{S \alpha}, i_{S \beta}$ \\
Phase Currents & $\mathrm{u}$ \\
Roltage & $i_{m R}$ \\
Rotor, Stator and Main Inductances & $i_{a}, i_{b}, i_{c}$ \\
Rotor and Stator Resistances & $L_{R}, L_{S}, L_{H}$ \\
Rotor and Stator Time constants & $R_{R}, R_{S}$ \\
Rotor Currents Scalar Components & $T_{R}, T_{S}$ \\
\hline
\end{tabular}

Table 2: Summary of Notations Used in this Research Paper 


\section{Appendix B}

\section{Induction Motor Parameters}

The induction motor used in this research paper is a single cage, three-phase, $60 \mathrm{~Hz}$, Y-connected induction motor. The rated values and the parameters of this motor are as follows:

\begin{tabular}{|c|c|}
\hline Rated power & $\mathrm{P}=5 \mathrm{HP}$ \\
\hline Rated voltage & $\begin{array}{l}\mathrm{V}=133 \mathrm{~V} \mathrm{rms} \\
\text { (phase) }\end{array}$ \\
\hline Rated current & $\mathrm{I}=12 \mathrm{~A} \mathrm{rms}$ \\
\hline Rated speed & 1800rpm \\
\hline Pole pairs & 2 \\
\hline Slip & 0.0278 \\
\hline Stator resistance (Rs) & $0.375 \Omega$ \\
\hline Rotor resistance $\left(\mathrm{R}_{\mathrm{R}}\right)$ & $0.405 \Omega$ \\
\hline Magnetizing inductance $(\mathrm{LH})$ & $77 \mathrm{mH}$ \\
\hline Stator leakage inductance (Los) & $2.63 \mathrm{mH}$ \\
\hline Stator inductance $(\mathrm{Ls}=\mathrm{L} \sigma \mathrm{s}+\mathrm{LH})$ & $79.63 \mathrm{mH}$ \\
\hline Rotor leakage inductance (Lof) & $2.63 \mathrm{mH}$ \\
\hline Rotor inductance $\left(L_{R}=L_{\sigma R}+L_{H}\right)$ & $79.63 \mathrm{mH}$ \\
\hline Leakage coefficient $\left(\sigma=1-L_{H} / L_{R} L_{s}\right)$ & 0.065 \\
\hline Rotor inertia & $19.36^{\star} 10^{-3} \mathrm{kgm}^{2}$ \\
\hline
\end{tabular}

These values are used in the electromagnetic equation of the induction motor as well as the mechanical equation and the control algorithm.

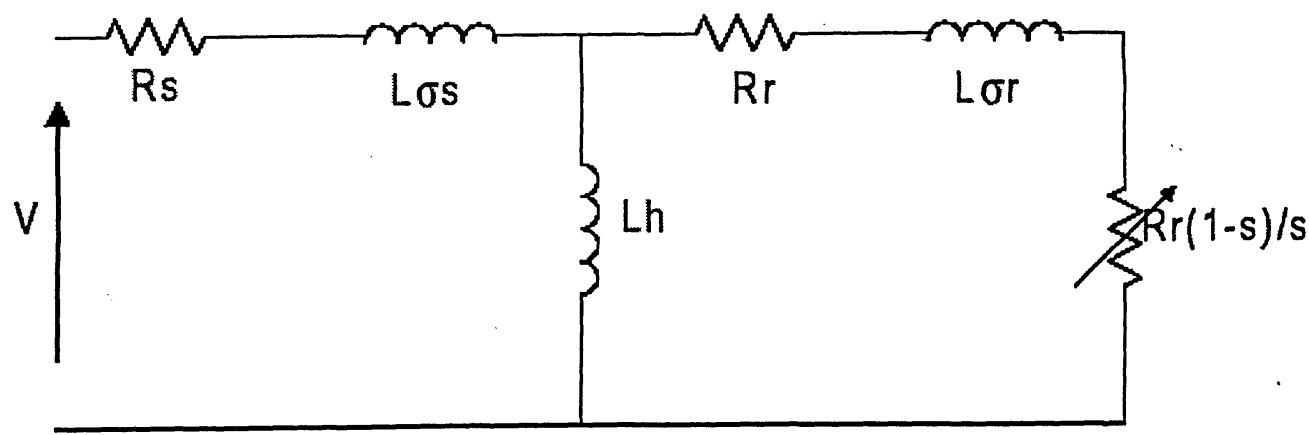

Figure 36: The Model of an Induction Motor 


\section{Appendix C}

\section{Characteristics of PID Control}

The proportional controller $(\mathrm{Kp})$ will have the effect on reducing the rise time a step response and will reduce, but not eliminate, the steady-state error. An integral control (Ki) will have the effect of eliminating the steady-state error, but it may make the transient response worse. A derivative control $(\mathrm{Kd})$ will have the effect of increasing the stability of the system, reducing the overshoot, and improving the transient response. Effects of each of controllers $\mathrm{Kp}, \mathrm{Kd}$, and $\mathrm{Ki}$ on a closed-loop system are summarized in the table shown below.

\begin{tabular}{|c|c|c|c|c|}
\hline CL RESPONSE & RISE TIME & OVERSHOOT & SETTLING TIME & S-S ERROR \\
\hline \hline $\mathrm{Kp}$ & Decrease & Increase & Small Change & Decrease \\
\hline $\mathrm{Ki}$ & Decrease & Increase & Increase & Eliminate \\
\hline $\mathrm{Kd}$ & Small Change & Decrease & Decrease & Small Change \\
\hline
\end{tabular}

Note that the correlations shown in the table may not be exactly accurate, because $\mathrm{Kp}, \mathrm{Ki}$, and $\mathrm{Kd}$ are dependent of each other. In fact, changing one of these variables can change the effect of the other two. For this reason, the table should only be used as a reference for determining the values of $\mathrm{Ki}, \mathrm{Kp}$ and $\mathrm{Kd}$. 


\section{Appendix D}

\section{Assembly Code for TMS320LF2407}

1) Main program-foc2.asm

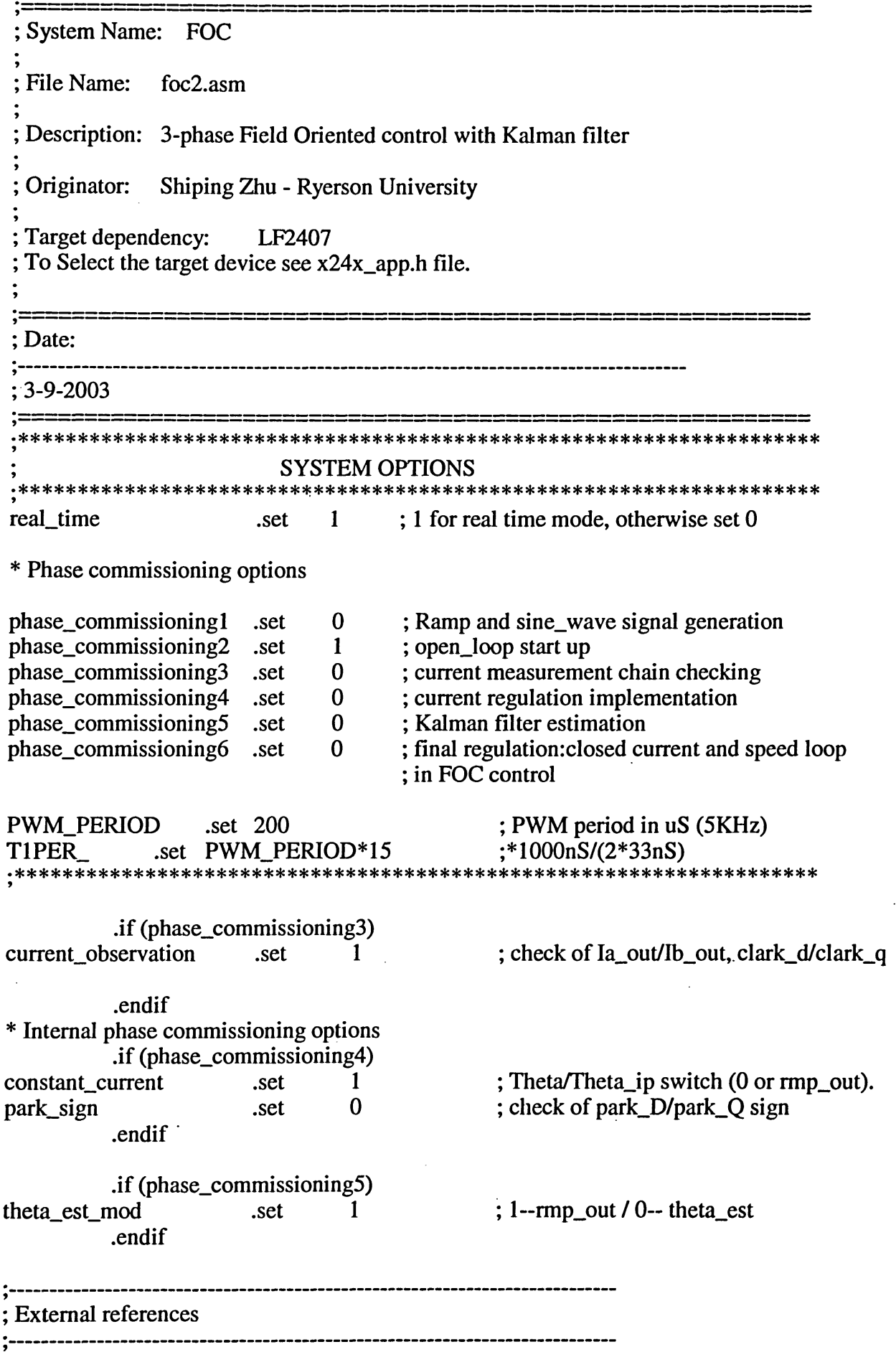




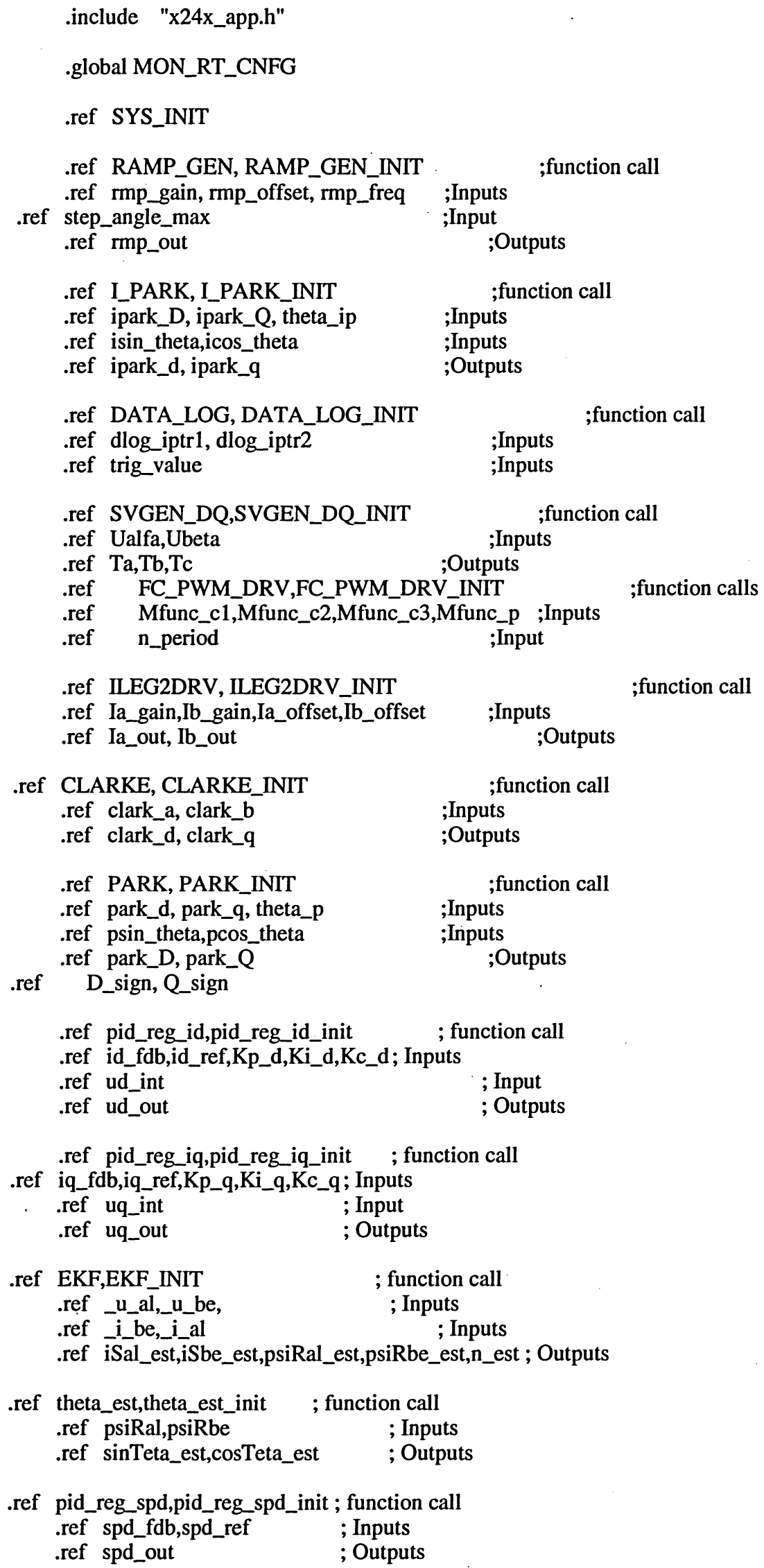




; Variable Declarations
.def GPR0
.bss GPR0,1 ;General purpose register
.bss my_iq_ref,1
.bss my_id_ref,1
.bss speed_reference, 1

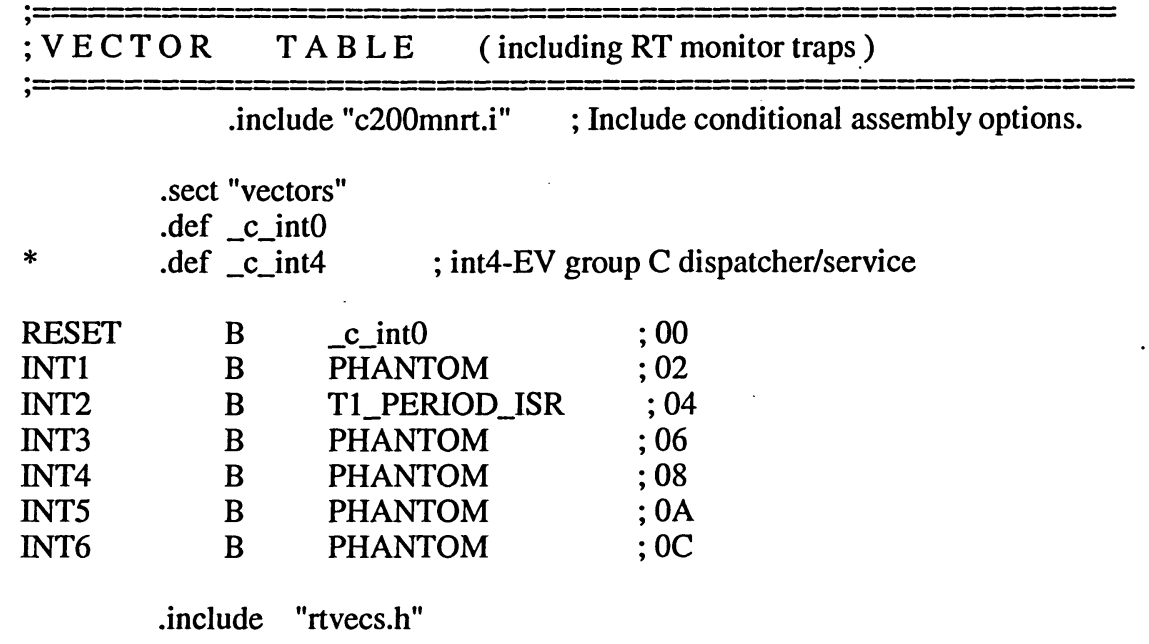

; Note : The above include line must be AFTER the user configurable ; vectors. Do not change the place where this line is included.

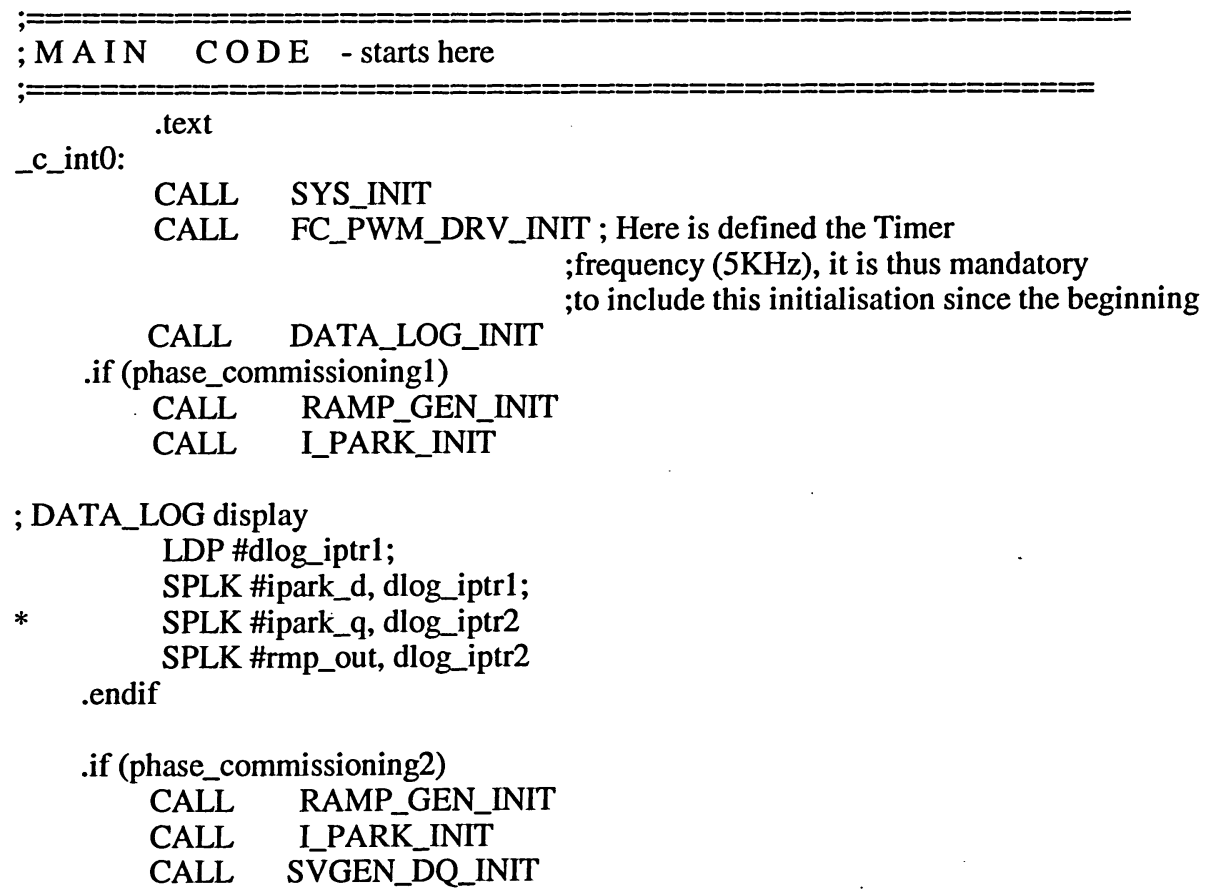

; DATA_LOG display

LDP \#dlog_iptr1;

SPLK \#ipark_d, dlog_iptr1;

* SPLK \#ipark_q, dlog_iptr2 


$$
\begin{array}{ll}
\text { * } & \multicolumn{2}{c}{\text { SPLK \#rmp_out, dlog_iptr2 }} \\
\text { SPLK \#Ta, dlog_iptr2 } \\
\text {.endif } \\
\text { if (phase_commissioning3) } \\
\text { CALL } & \text { RAMP_GEN_INIT } \\
\text { CALL } & \text { I_PARK_INIT } \\
\text { CALL } & \text { SVGEN_DQ_INIT } \\
\text { CALL } & \text { ILEG2DRV_INIT } \\
\text { CALL } & \text { CLARKE_INIT } \\
\text { CALL } & \text { PARK_INIT }
\end{array}
$$
CALL RAMP_GEN_INIT
CALL I_PARK_INIT
CALL SVGGEN_DQ INIT
CALL ILEG2DRV_INIT
CALL CLARKE_INIT
CALL PARK_INIT

\section{; DATA_LOG display}

\section{LDP \#dlog_iptr1;}

SPLK \#Ia_out, dlog_iptr1;

$\begin{array}{ll}* & \text { SPLK \#Ib_out, dlog_iptr2 } \\ * & \text { SPLK \#clark_d, dlog_iptr1 }\end{array}$

SPLK \#clark_q, dlog_iptr2

* $\quad$ SPLK \#park_D, dlog_iptr1

* SPLK \#park_Q, dlog_iptr2

.endif

if (phase_commissioning4)
CALL RAMP_GEN_INIT
CALL I_PARK_INIT
CALL SV̄GEN_DQ_INIT
CALL ILEG2DRV_INIT
CALL CLARKE_INIT
CALL PARK_INIT
* CALL QEP_THETA_DRV_INIT
CALL pid_reg_id_init
CALL pid_reg_iq_init

* CALL SPEED_FRQ_INIT

; DATA_LOG display

LDP \#dlog_iptr1; this is inserted by Shiping SPLK \#rmp_out, dlog_iptr1;

.endif splk \#Ia_out, dlog_iptr2

if (phase_commissioning5)
CALL RAMP_GEN_INIT
CALL I_PARK_INIT
CALL SVGEN_DQ_INIT
CALL ILEG2DRV_INIT
CALL CLARKE_INIT
CALL PARK_INIT
CALL EKF_INIT
CALL pid_reg_id_init
CALL pid_reg_iq_init
CALL theta_est_init

; DATA_LOG display

.endif

if (phase_commissioning6)
CALL I_PARK_INIT
CALL SVGGEN_DQ_INIT
CALL ILEG2DRV_INIT
CALL CLARKE_INIT 

CALL PARK_INIT
CALL EKF_INIT
CALL pid_reg_id_init
CALL pid_reg_iq_init
CALL theta_est_init
CALL pid_reg_spd_init

\section{; DATA_LOG display \\ .endif}

;---Real Time option .if (real_time) .endif

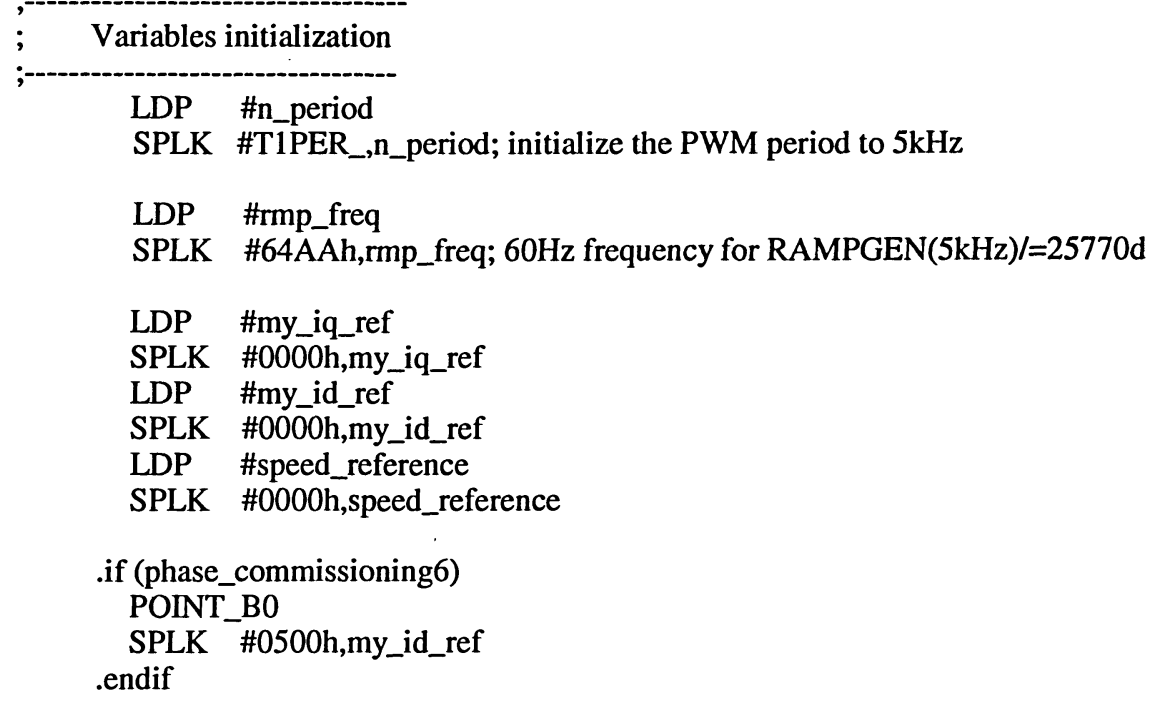


SPLK \#OFFFFh, IFR $\quad$;Clear any pending Ints EINT ;Enable global Ints

POINT_B0

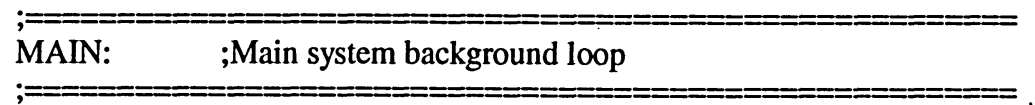

M_1 NOP

NOP

NOP

CLRC XF

B MAIN

; Routine Name: T1_PERIOD_ISR $\quad$ Routine Type: ISR

; Description:

;

; Originator: Shiping Zhu - Ryerson University

;

;; Last Update: 20-Apr 03

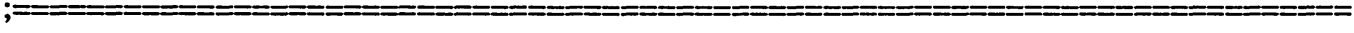

$==$

T1_PERIOD_ISR:

;Context save regs

MAR *,AR1 $\quad$;AR1 is stack pointer

MAR ;skip one position

SST \#1, *+ ;save ST1

SST \#0,*+ ;save ST0

$\mathrm{SACH} \quad *_{+} \quad$;save acc high

SACL $* \quad$;save acc low

;NOTE: should use "read-modify-write" to clear Int flags \& not SPLK!

POINT_EV

SPLK \#OFFFFh,IFRA ; Clear all Group A interrupt flags (T1 ISR)

SETC XF

SETC SXM ; set sign extension mode

CLRC OVM ; clear overflow mode

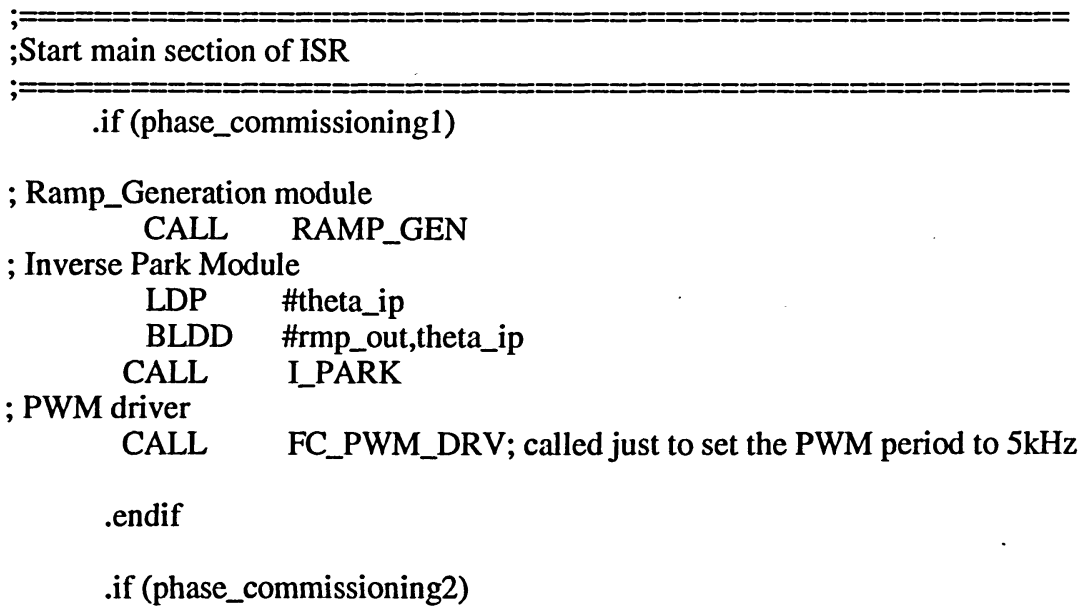


; Ramp generation module

CALL RAMP_GEN

; Inverse-Park module

LDP \#theta_ip

BLDD \#rmp_out,theta_ip

CALL I_PARK

; Space-Vector DQ module

LDP \#Ualfa

BLDD \#ipark_d,Ualfa

BLDD \#ipark_q,Ubeta

CALL SVGEN_DQ

; PWM driver
LDP \#Mfunc_cl
BLDD \#Ta,Mfunc_c1
BLDD \#Tb,Mfunc_c2
BLDD \#Tc,Mfunc_c3
CALL FC_PWM_DRV

.endif

.if (phase_commissioning3)

; Current leg measurement, Ileg2drv module

$$
\text { CALL ILEG2DRV }
$$

; Clarke module

$$
\begin{array}{ll}
\text { LDP } & \text { \#clark_a } \\
\text { BLDD } & \text { \#Ia_out,clark_a } \\
\text { BLDD } & \text { \#Ib_out,clark_b } \\
\text { CALL } & \text { CLARKE }
\end{array}
$$

; PARK module
LDP
BLDD \#clark_d,park_d
BLDD \#clark_q,park_q
BLDD \#rmp_out,theta_p
CALL PARK

; Ramp generation module

$$
\text { CALL RAMP_GEN }
$$

; Inverse-Park module

$$
\begin{array}{ll}
\text { LDP } & \text { \#theta_ip } \\
\text { BLDD } & \text { \#rmp_out,theta_ip } \\
\text { CALL } & \text { I_PARK }
\end{array}
$$

; Space-Vector DQ module

$$
\text { LDP \#Ualfa }
$$

BLDD \#ipark_d,Ualfa

BLDD \#ipark_q,Ubeta

; PWM driver

CALL SVGEN_DQ
LDP \#Mfunc_c1
BLDD \#Ta,Mfunc_c1
BLDD \#Tb,Mfunc_c2
BLDD \#Tc,Mfunc_c3
CALL FC_PWM_DRV

.endif

.if (phase_commissioning4)

; Current leg measurement, Ileg2drv module CALL ILEG2DRV

; Clarke module

LDP \#clark_a

BLDD \#Ia_out,clark_a 


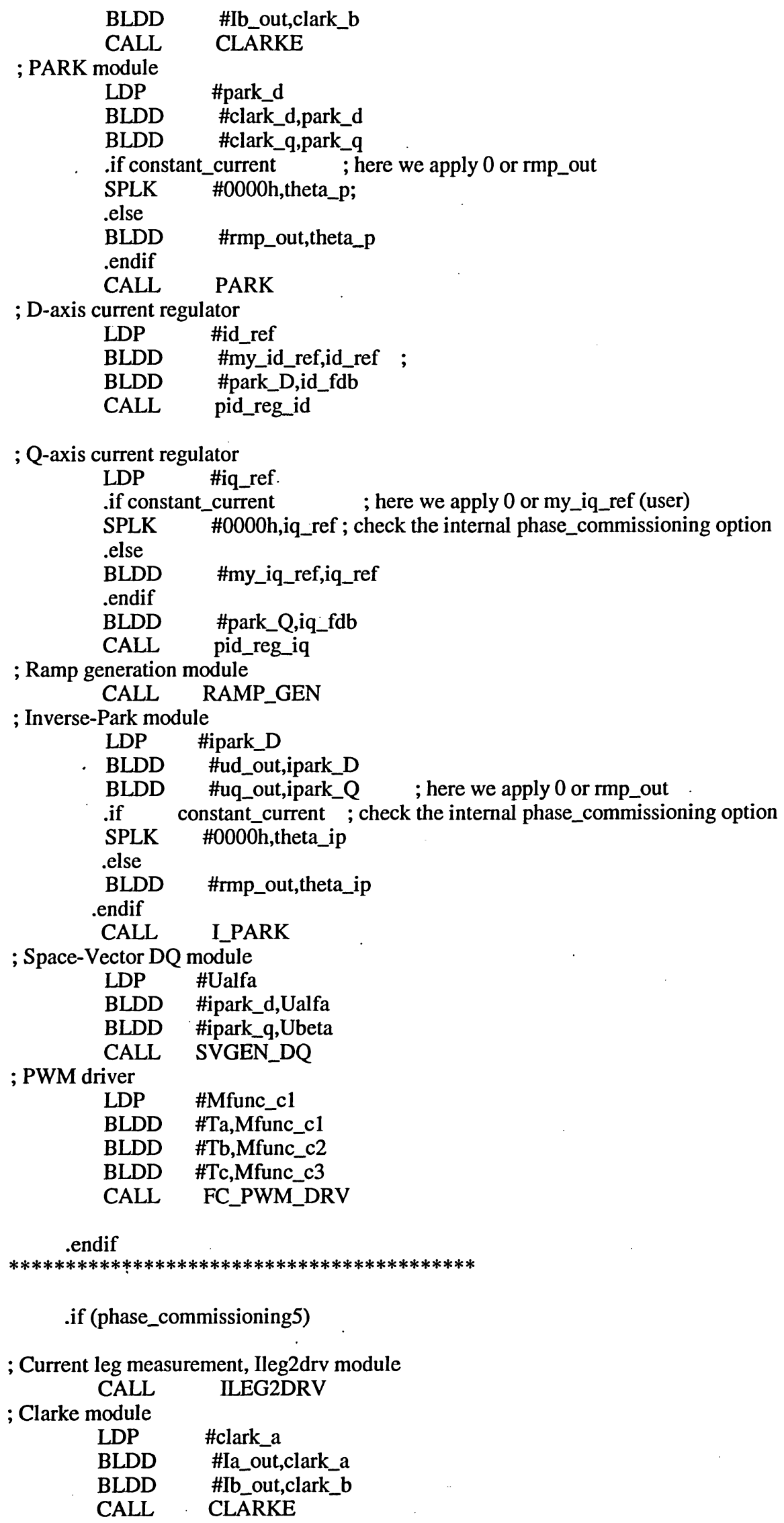


; EKF module

$$
\begin{array}{lc}
\text { LDP } & \text { \#_u_al } \\
\text { BLDD } & \text { \#ipark_d,_u_al } \\
\text { BLDD } & \text { \#ipark_q,_u_be } \\
\text { BLDD } & \text { \#clark_d,_i_al } \\
\text { BLDD } & \text { \#clark_q,_i_be } \\
\text { CALL } & \text { EKF }
\end{array}
$$

; PARK module

LDP \#park_d

BLDD \#iSal_est,park_d

BLDD \#iSbe_est,park_q

.if theta_est_mod $\quad$; here we connect rmp_out or theta_est_mod

BLDD \#rmp_out,theta_p ; check the internal phase_commissioning option

.else

BLDD \#sinTeta_est,psin_theta

BLDD \#cosTeta_est,pcos_theta

.endif

CALL PARK

; theta_est
LDP \#psiRal
BLDD \#psiRal_est,psiRal
BLDD \#psiRbe_est,psiRbe
CALL theta_est

; D-axis current regulator

$$
\begin{array}{ll}
\text { LDP } & \text { \#id_ref } \\
\text { BLDD } & \text { \#my_id_ref,id_ref } \\
\text { BLDD } & \text { \#park_D,id_fdb } \\
\text { CALL } & \text { pid_reg_id }
\end{array}
$$

; Q-axis current regulator

$$
\begin{array}{ll}
\text { LDP } & \text { \#iq_ref } \\
\text { BLDD } & \text { \#my_iq_ref,iq_ref } \\
\text { BLDD } & \text { \#park_Q,iq_fdb } \\
\text { CALL } & \text { pid_reg_iq }
\end{array}
$$

; Ramp generation module

$$
\text { CALL RAMP_GEN }
$$

; Inverse-Park module

$$
\begin{array}{ll}
\text { LDP } & \text { \#ipark_D } \\
\text { BLDD } & \text { \#ud_out,ipark_D } \\
\text { BLDD } & \text { \#uq_out,ipark_Q }
\end{array}
$$

if theta_est_mod ; here we connect rmp_out or theta_est_mod

BLDD \#rmp_out,theta_ip ; check the internal phase_commissioning option

.else

BLDD \#sinTeta_est,isin_theta

BLDD \#cosTeta_est,icos_theta

.endif

CALL I_PARK

; Space-Vector DQ module

LDP \#Ualfa

BLDD \#ipark_d,Ualfa

BLDD \#ipark_q,Ubeta

; PWM driver

CALL SVGEN_DQ
LDP \#Mfunc c1
BLDD \#Ta,Mfunc_c1
BLDD \#Tb,Mfunc_c2
BLDD \#Tc,Mfunc_c3
CALL FC_PWM_DRV

.endif 
.if (phase_commissioning6)

; Current leg measurement, Ileg2drv module

; Clarke module $\begin{array}{ll}\text { LDP } & \text { \#clark_a } \\ \text { BLDD } & \text { \#Ia_out,clark_a } \\ \text { BLDD } & \text { \#Ib_out,clark_b }\end{array}$

BLDD \#Ib_out,cl

; SPEED REGULATION

LDP \#spd_ref

bldd \#speed_reference,spd_ref

bldd \#n_est,spd_fdb

; EKF module

CALL pid_reg_spd

$$
\begin{array}{ll}
\text { LDP } & \text { \#_u_al } \\
\text { BLDD } & \text { \#ipark_d,_u_al } \\
\text { BLDD } & \text { \#ipark_q,_u_be } \\
\text { BLDD } & \text { \#clark_d,_i_al } \\
\text { BLDD } & \text { \#clark_q,_i_be } \\
\text { CALL } & \text { EKF }
\end{array}
$$

; PARK module

LDP \#park_d

BLDD \#iSal_est,park_d

BLDD \#iSbe_est,park_q

BLDD \#sinTeta_est,psin_theta

BLDD \#cosTeta_est,pcos_theta

; theta_est

CALL PARK

$$
\begin{array}{ll}
\text { LDP } & \text { \#psiRal } \\
\text { BLDD } & \text { \#psiRal_est,psiRal } \\
\text { BLDD } & \text { \#psiRbe_est,psiRbe } \\
\text { CALL } & \text { theta_est }
\end{array}
$$

; D-axis current regulator

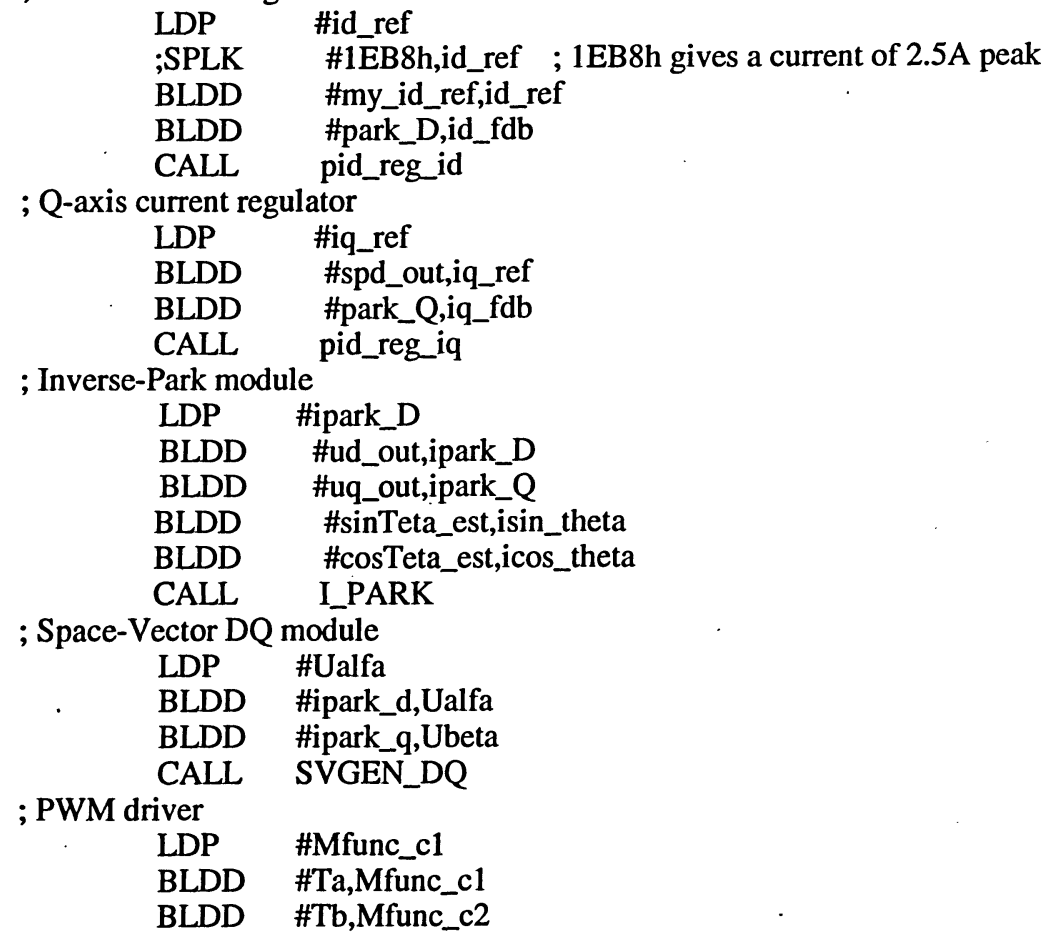

$\begin{array}{ll}\text { LDP } & \text { \#id_ref } \\ \text {;SPLK } & \text { \#1EB8h,id_ref ; 1EB8h gives a current of 2.5A peak } \\ \text { BLDD } & \text { \#my_id_ref,id_ref } \\ \text { BLDD } & \text { \#park_D,id_fdb } \\ \text { CALL } & \text { pid_reg_id }\end{array}$

; Q-axis current regulator 
BLDD \#Tc,Mfunc_c3

CALL FC_PWM_DRV

.endif

;Allow variable viewing via $\mathrm{DAC}$ o/ps

CALL DATA_LOG

CALL DAC_VIEW_DRV

;End main section of ISR

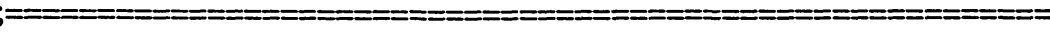

;Context restore regs

END_ISR:

POINT_PG0

MAR *, AR1 ;make stack pointer active

LACL $\quad *_{-} \quad$;Restore Acc low

ADDH $\quad *_{-} \quad$;Restore Acc high

LST \#0, *- ;load ST0

LST \#1, * ;load ST1

EINT

RET

; I I R - PHANTOM
;
; Description: Dummy ISR, used to trap spurious interrupts.
; Modifies:
; Last Update: 11-03-03
;=============================================================
PHANTOM B PHANTOM




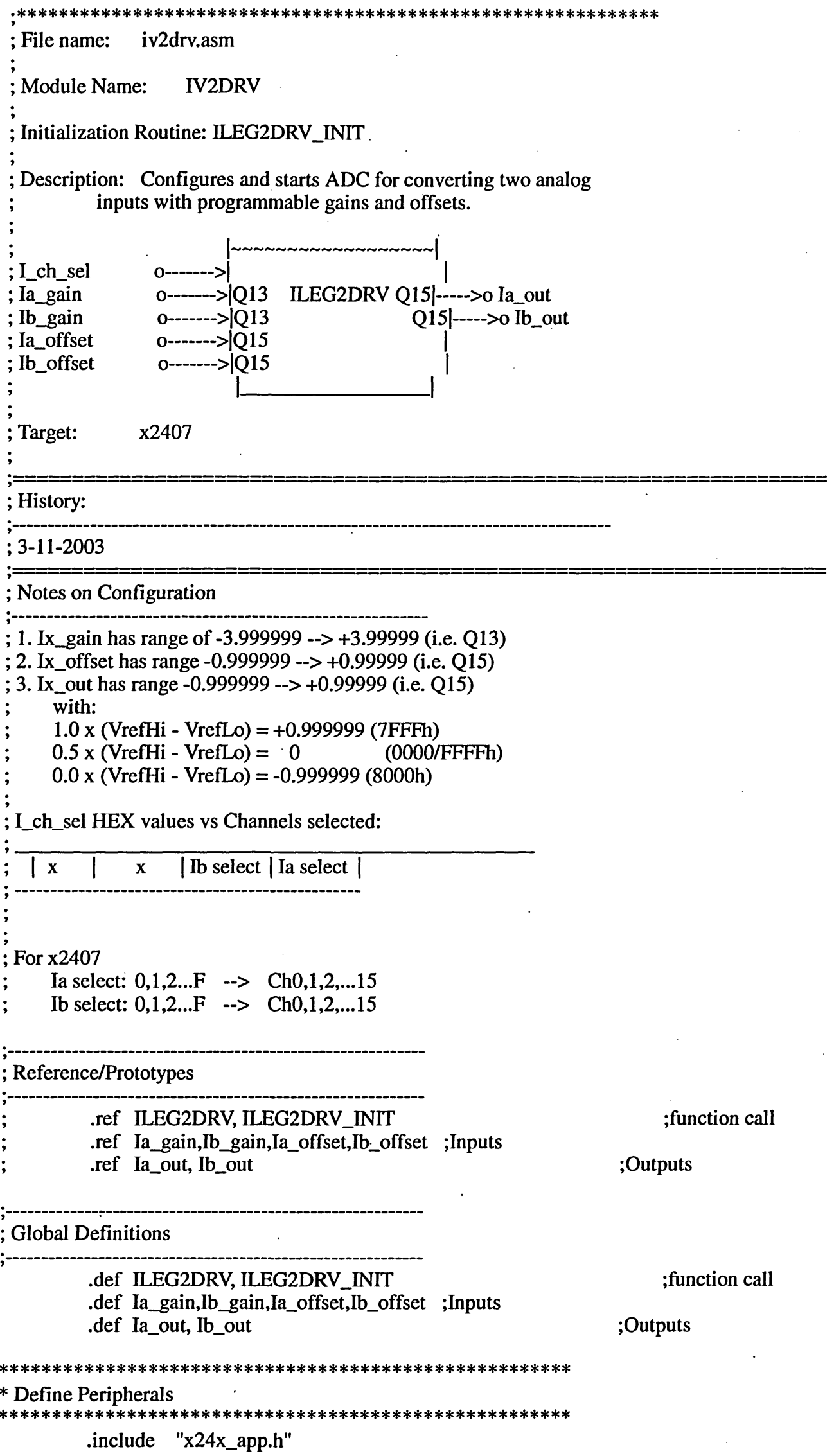




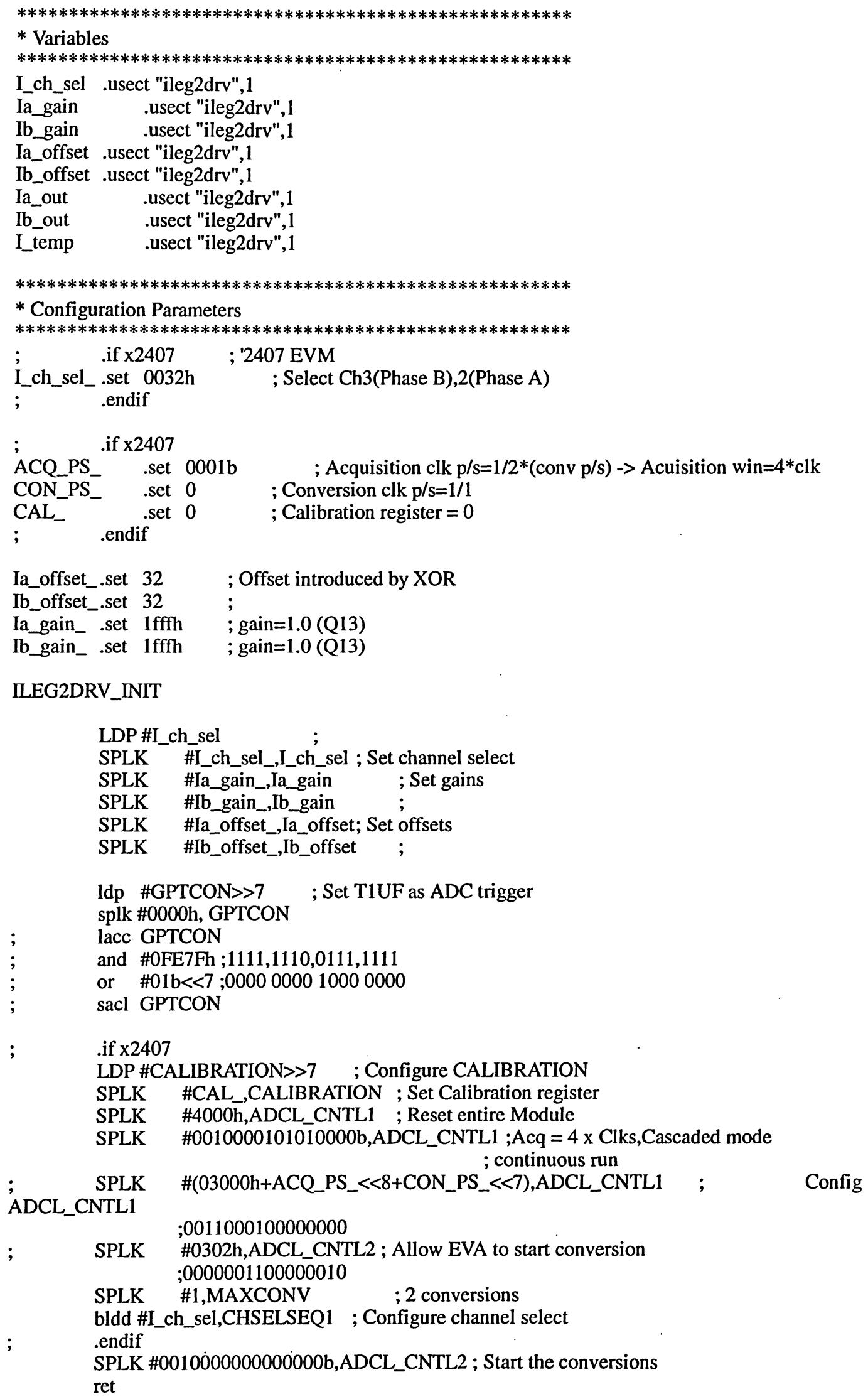

ILEG2DRV_INIT

LDP \#I_ch_sel
SPLK \#I_ch_sel_,I_ch_sel ; Set channel select
SPLK \#Ia_gain_,Ia_gain $\quad$; Set gains
SPLK \#Ib_gain_Ib_gain $\quad$;
SPLK \#Ia_offset_,Ia_offset; Set offsets
SPLK \#Ib_offset_Ib_offset ;

Idp \#GPTCON $\gg>7$; Set T1UF as ADC trigger splk \#0000h, GPTCON

; $\quad$ lacc GPTCON

and \#0FE7Fh ; 1111,1110,0111,1111

or $\# 01 \mathrm{~b}<<7 ; 0000000010000000$

sacl GPTCON

; $\quad$.if $\times 2407$

LDP \#CALIBRATION $>>7$; Configure CALIBRATION

SPLK \#CAL_CALIBRATION ; Set Calibration register

SPLK \#4000h,ADCL_CNTL1 ; Reset entire Module

SPLK \#0010000101010000b,ADCL_CNTL1 ;Acq = 4 x Clks,Cascaded mode ; continuous run

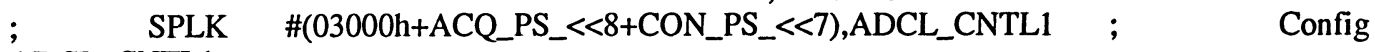

ADCL_CNTL1 ;0011000100000000

; $\quad$ SPLK $\quad \# 0302 \mathrm{~h}, \mathrm{ADCL} \_C N T L 2$; Allow EVA to start conversion ;0000001100000010

SPLK \#1,MAXCONV ;2 conversions

bldd \#I_ch_sel,CHSELSEQ1 ; Configure channel select

; $\quad$.endif

SPLK \#0010000000000000b,ADCL_CNTL2 ; Start the conversions ret 


\section{ILEG2DRV:}

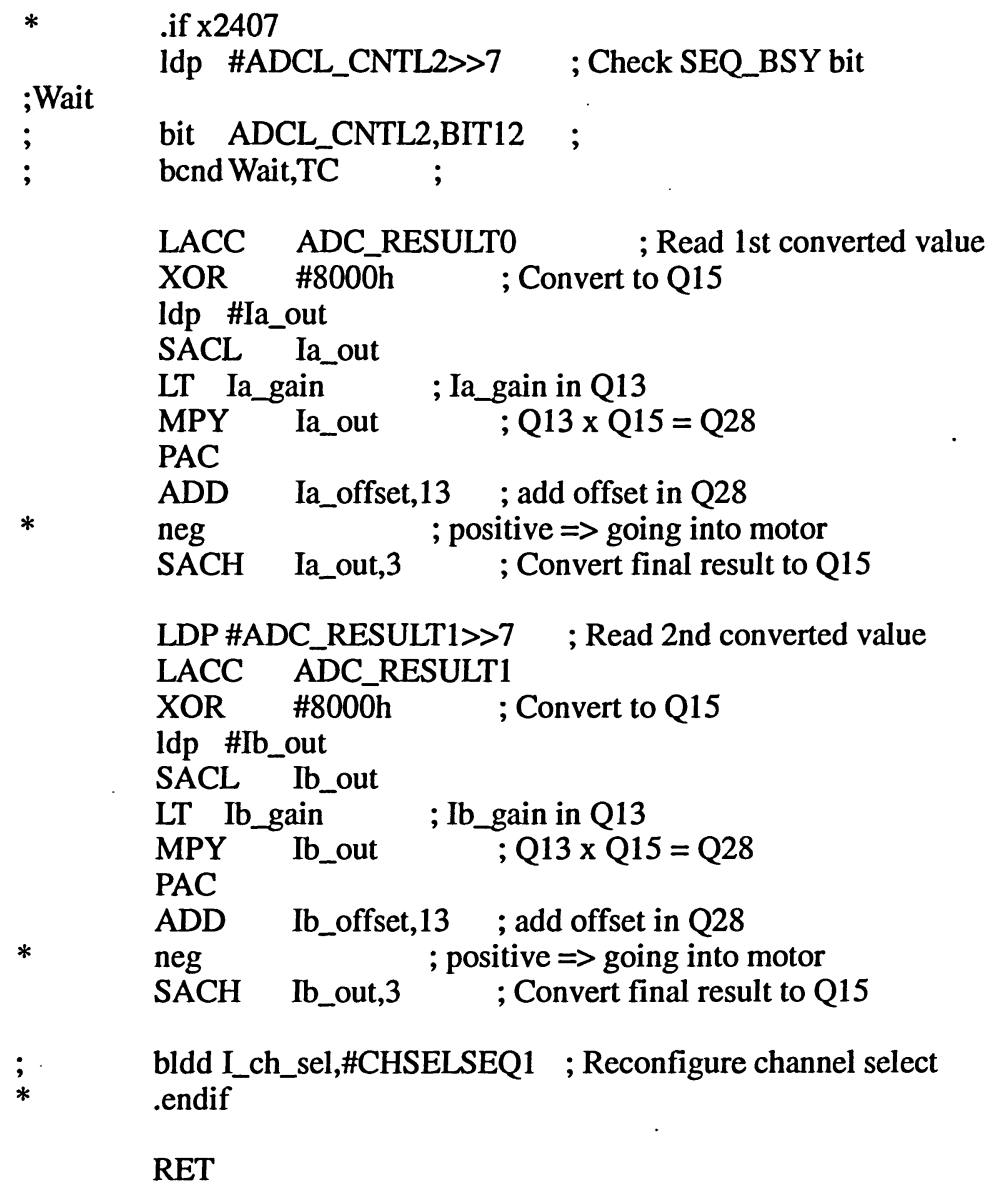

\footnotetext{
; File Name: park.asm

; Module Name: PARK

; Description: This transformation converts vectors in balanced 2-phase orthogonal stationary system into orthogonal rotating reference frame.

$$
\text { id }=\text { ialfa } * \text { cos_teta }+ \text { ibeta } * \text { sin_teta }
$$$$
\mathrm{iq}=\text {-ialfa } * \sin \_ \text {teta }+ \text { ibeta } * \text { cos_teta }
$$

park_d

park_q

theta_p

;or psin_theta

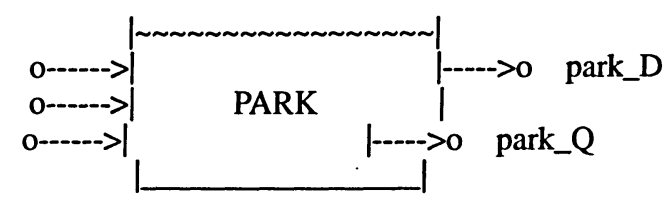

pcos_theta

Note: $0<$ theta_p $<7$ FFFh (i.e. equivalent to $0<$ theta_p $<360$ deg )
} 


\begin{tabular}{|c|c|}
\hline $\begin{array}{l}\text {; Target dependency:C } 2 \mathrm{xx} \text { core only } \\
;\end{array}$ & \\
\hline ; History: & \\
\hline ; 3-11-2003 & \\
\hline 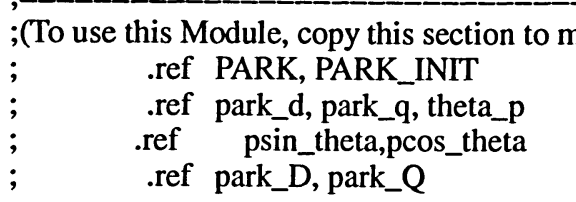 & $\begin{array}{l}\text { em file) } \\
\quad \text {;function call } \\
\text {;Inputs } \\
\text {;Inputs } \\
\quad ; \text { Outputs }\end{array}$ \\
\hline $\begin{array}{l}\text {;Module definitions for external reference. } \\
\text {.def PARK, PARK_INIT } \\
\text {.def park_d, park_q, theta_p } \\
\text {.def psin_theta,pcos_theta } \\
\text {.def park_D, park_Q } \\
\text {.def } \text { D_sign, Q_sign }\end{array}$ & $\begin{array}{l}\text {;function call } \\
\text {;Inputs } \\
\text {;Inputs } \\
\text {;Outputs } \\
\text {;D,Q sign }\end{array}$ \\
\hline
\end{tabular}

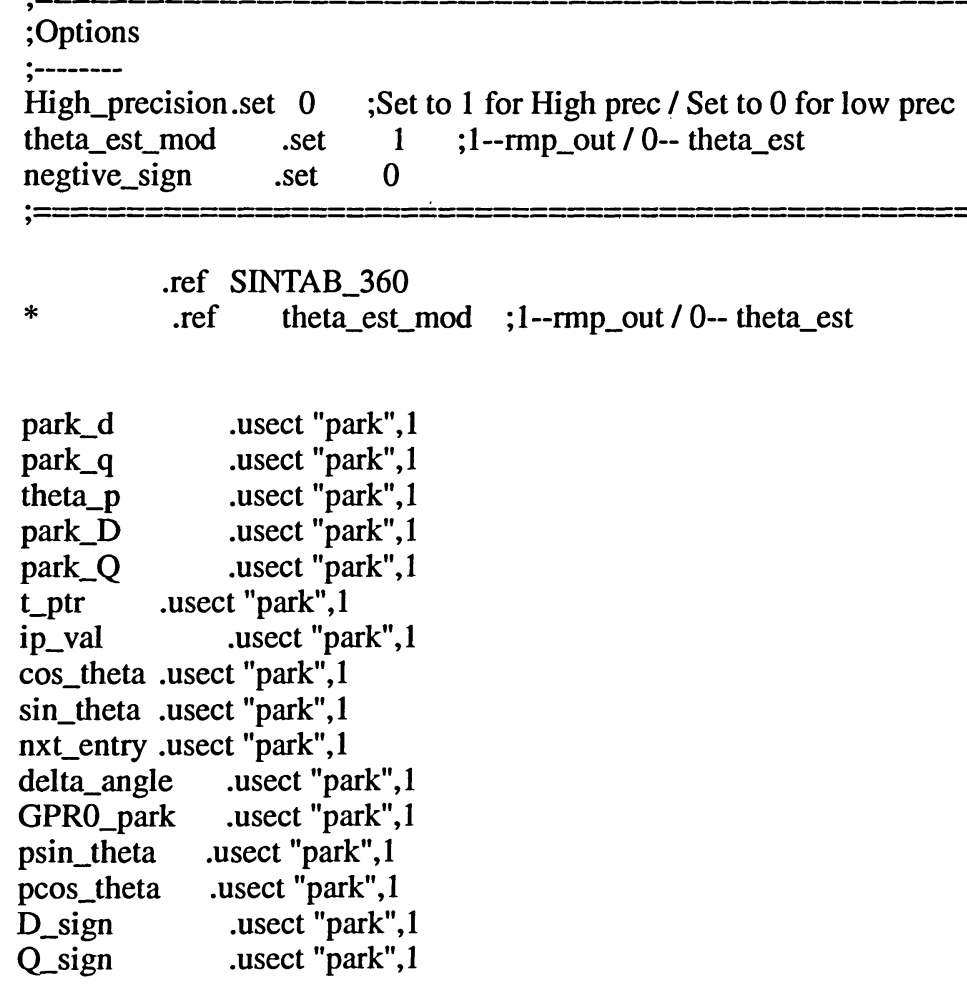

RET

\section{PARK:}

* = ====-==-==-===-

.if theta_est_mod 
;Calculate Cos(theta_p)

;--- High_precision option -------

.if (High_precision) ;see park for its definition

;Higher precision using look-up + interpolation method

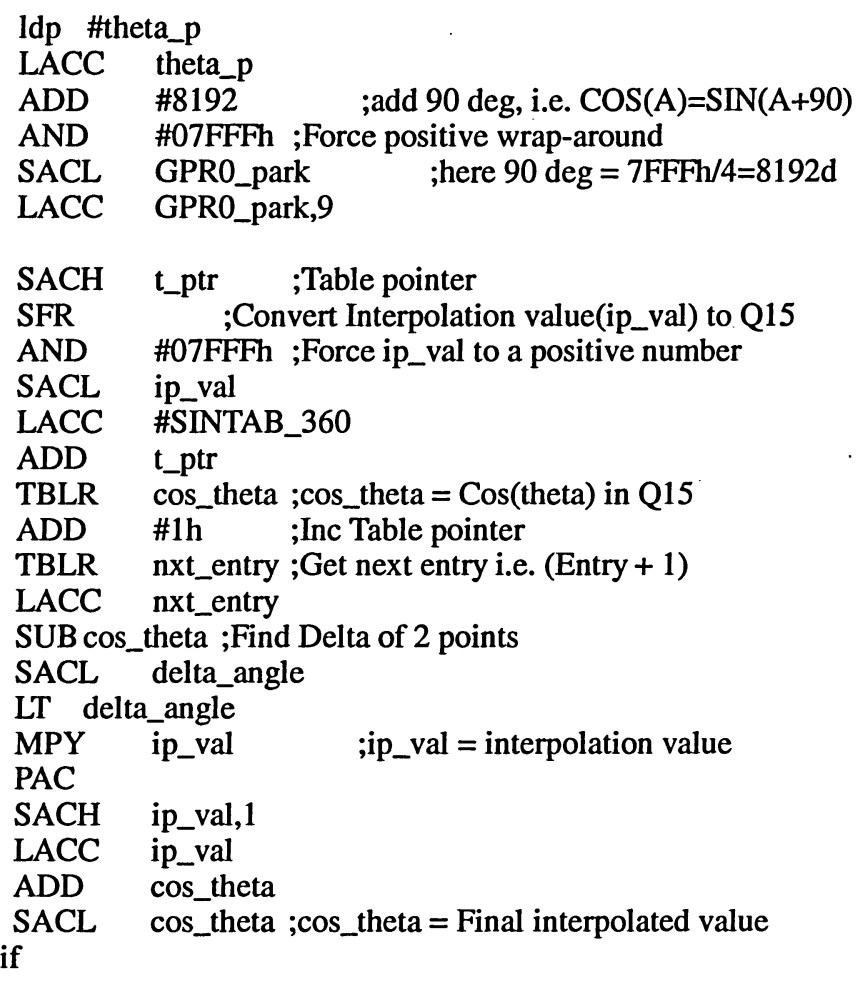

;--- Normal precision option -------

.if (High_precision != 1)

;Normal precision with simple 256 word look-up

ldp \#theta_p

LACC theta_p

ADD \#8192 ;add 90 deg, i.e. $\operatorname{COS}(A)=\operatorname{SIN}(A+90)$

AND \#07FFFh ;Force positive wrap-around

SACL . GPR0_park ;here $90 \mathrm{deg}=7 \mathrm{FFFh} / 4$

LACC GPR0_park,9

SACH t_ptr

LACC \#SINTAB_360

ADD t_ptr .endif

TBLR cos_theta $; \cos$ _theta $=\operatorname{Cos}($ theta_p) in Q15

;Calculate Sin(theta_p)

;--- High_precision option --------

.if (High_precision)

;Higher precision using look-up + interpolation method

LACC theta_p,9

SACH t_ptr ;Table pointer

SFR ;Convert Interpolation value(ip_val) to Q15

AND \#07FFFh ;Force ip_val to a positive number

SACL ip_val

LACC \#SINTAB_360

ADD t_ptr 


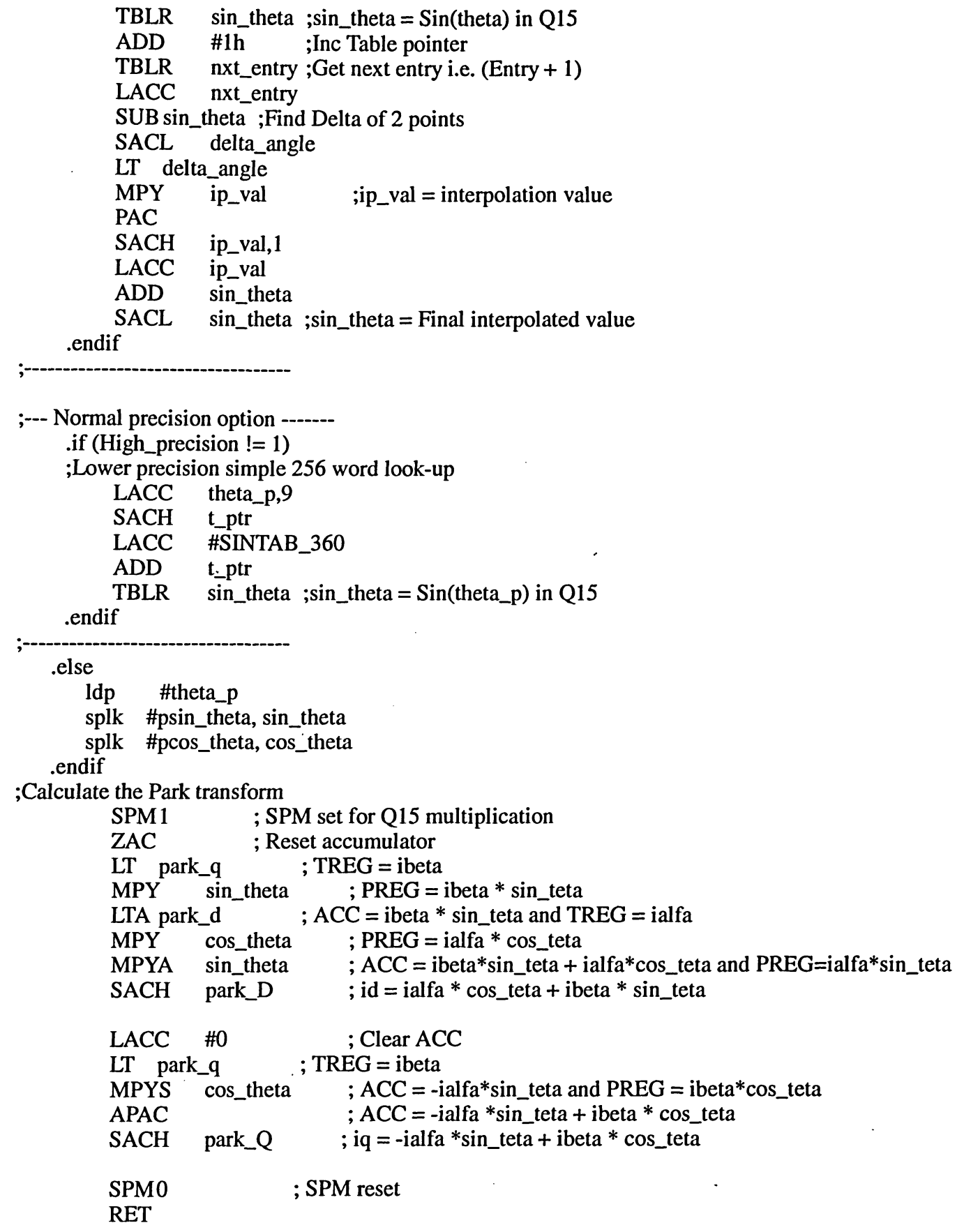




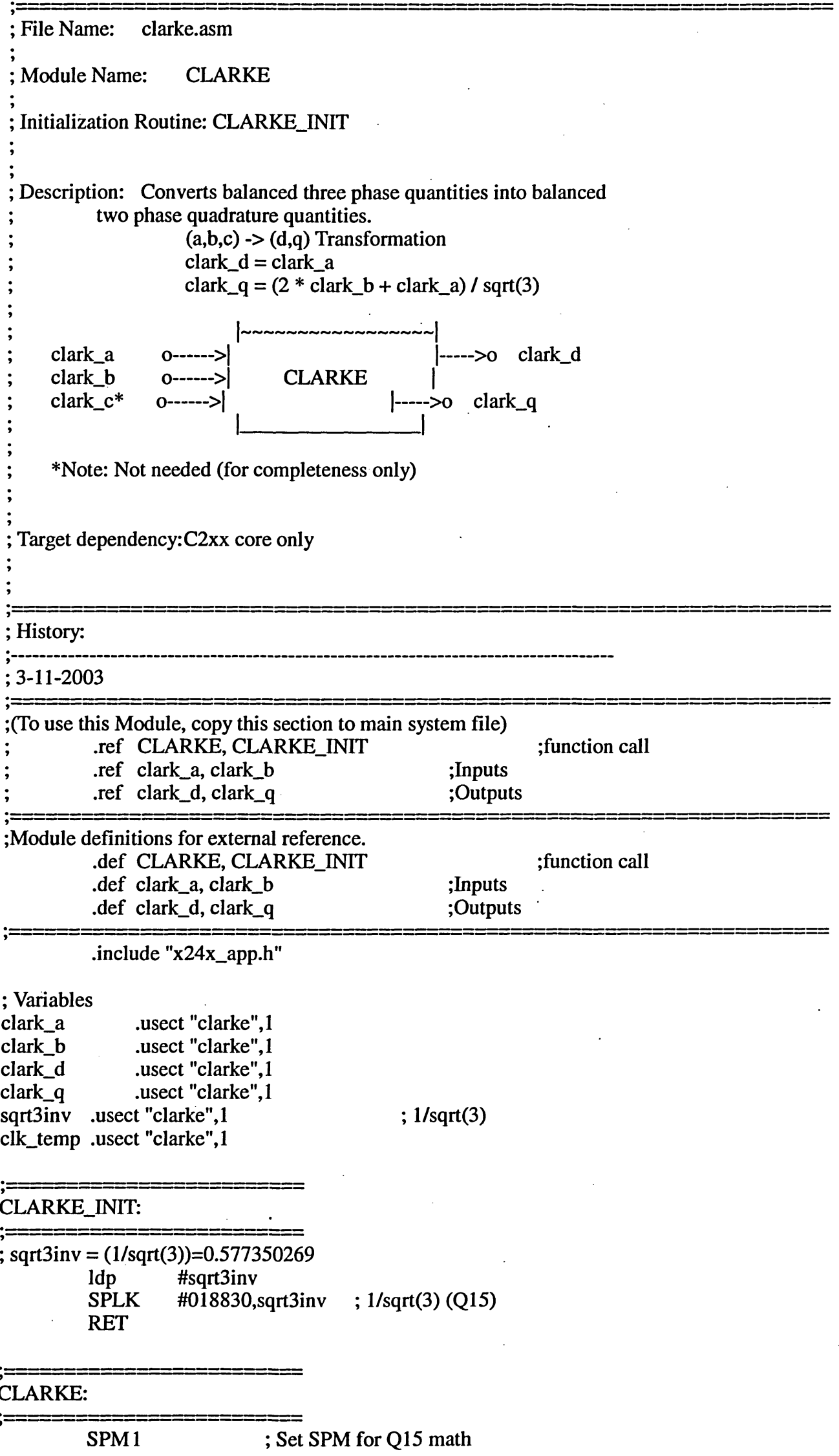


SETC SXM ; Sign extension mode on

;clark_d = clark_a

ldp \#clark_a

LACC clark_a ; ACC $=$ clark_a

SACL clark_d $\quad ;$ clark_d $=$ clark_a

;clark_q $=(2 *$ clark_b + clark_a $) / \operatorname{sqrt}(3)$

\begin{tabular}{|c|c|c|}
\hline \multicolumn{3}{|r|}{; ACC $=$ clark_a/2 } \\
\hline ADD & clark_b & ; $A C C=$ clark_a $/ 2+$ clark_b \\
\hline SACL & clk_temp & $;$ clk_temp $=$ clark_a/2 + clark_b \\
\hline & temp & ; TREG = clark_a/2 + clark_b \\
\hline MPY & sqrit3inv & ; PREG $=($ clark_a/2 + clark_b $) *(1 /$ sqrt(3) $)$ \\
\hline 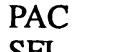 & & $;$ ACC $=($ clark_a $/ 2+$ clark_b $) *(1 /$ sqrt $(3))$ \\
\hline SFL & & $; \mathrm{ACC}=($ clark_a + clark_b*2)*(1/sqrt (3)) \\
\hline $\begin{array}{l}\text { SACH } \\
\text { SPMO }\end{array}$ & clark_q & $\begin{array}{l}\text {; clark_q = (clark_a + clark_b*2)*(1/sqrt(3)) } \\
\quad ; \text { SPM reset }\end{array}$ \\
\hline
\end{tabular}

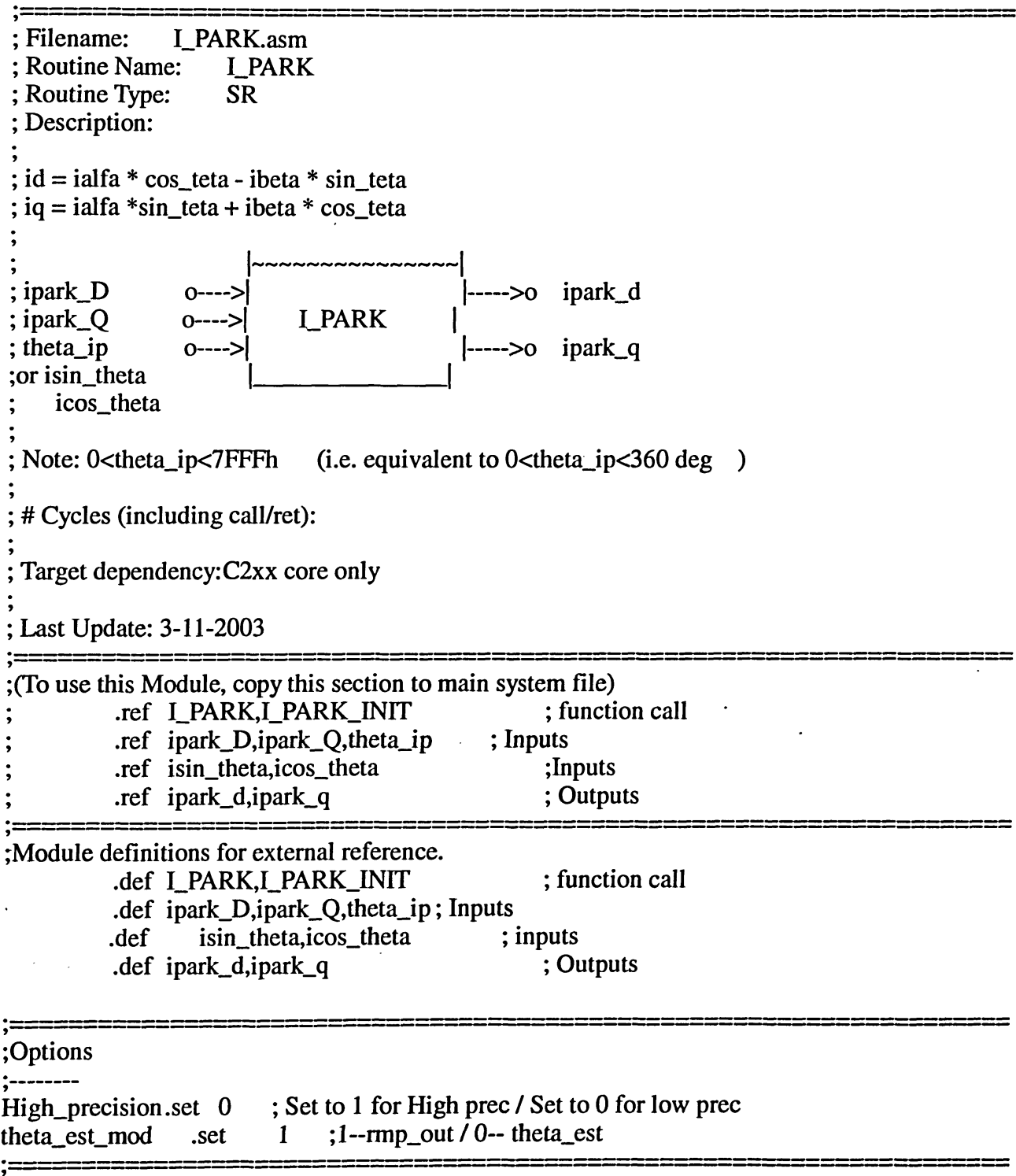




$$
\begin{aligned}
& \text {.ref SINTAB_360; } \text {. Sine table } \\
& \text { ref theta_est_mod }
\end{aligned}
$$



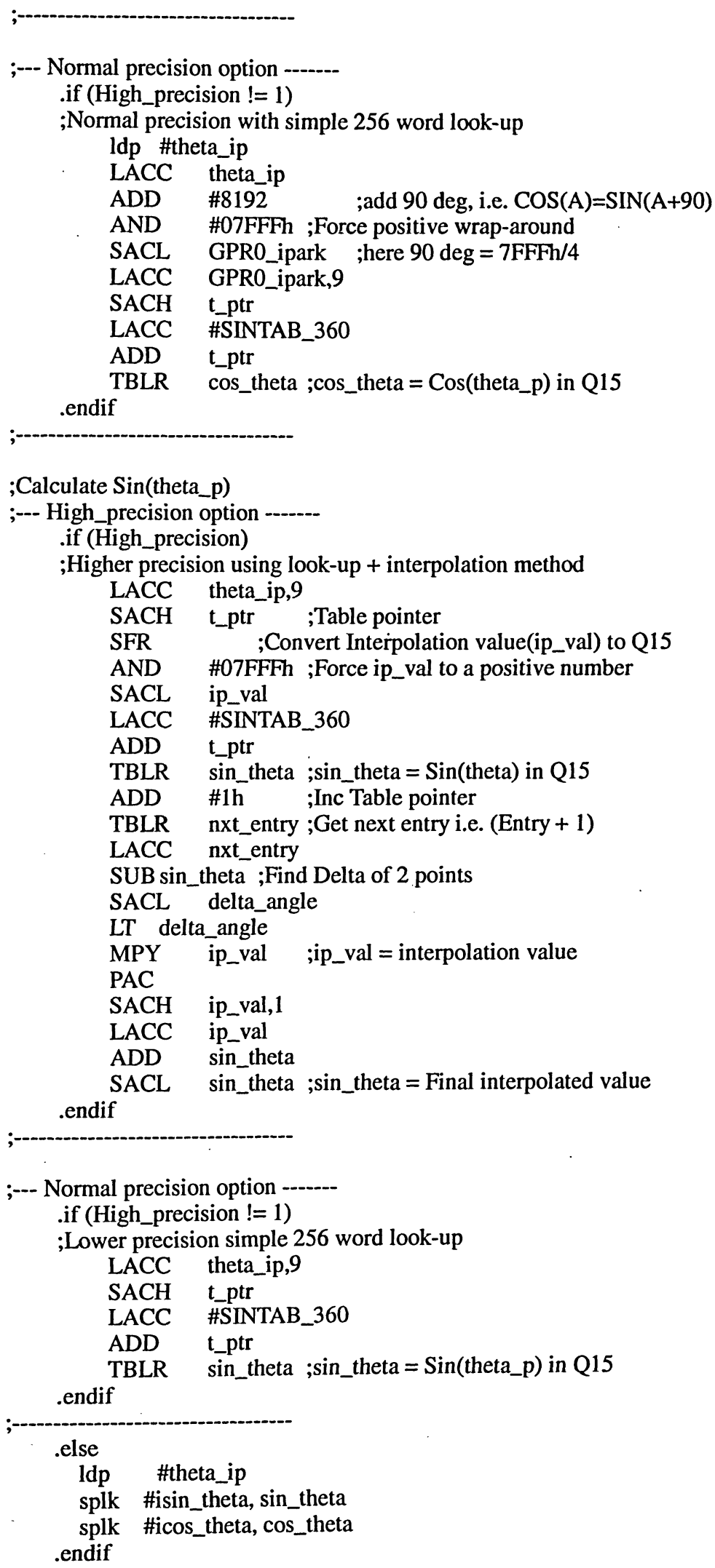

;Calculate the Inverse Park transform 
;park_q $=$ ipark_Q $*$ cos_theta + ipark_D $*$ sin_theta

LACC \#0 ; Clear ACC

LT ipark_D ; TREG = Udref

MPY sin_theta ; PREG $=$ Udref * sin_theta

LTA ipark_Q ; ACC $=$ Udref*sin_theta and TREG=Uqref

MPY cos_theta ; PREG $=$ Uqref $*$ cos_teta

TREG=Uqref* ${ }^{*}$ in_theta

MPYA sin_theta ; ACC $=$ Uqref*cos_theta + Udref*sin_theta and

SACH ipark_q ; Ubeta $=$ Uqref*cos_theta + Udref*sin_theta

;park_d $=$ ipark_D $*$ cos_theta - ipark_Q $*$ sin_theta

LACC \#0 ; Clear ACC

LT ipark_D ; TREG $=$ Udref

MPYS cos_theta ;ACC $=-$ Uqref*sin_theta and PREG $=$ Udref*cos_theta

APAC $\quad ;$ ACC $=-$ Uqref*sin_theta + Udref*cos_theta

SACH ipark_d ; Ualfa $=-$ Uqref $^{*}$ sin_theta + Udref*cos_theta

SPM0 ; SPM reset

RET

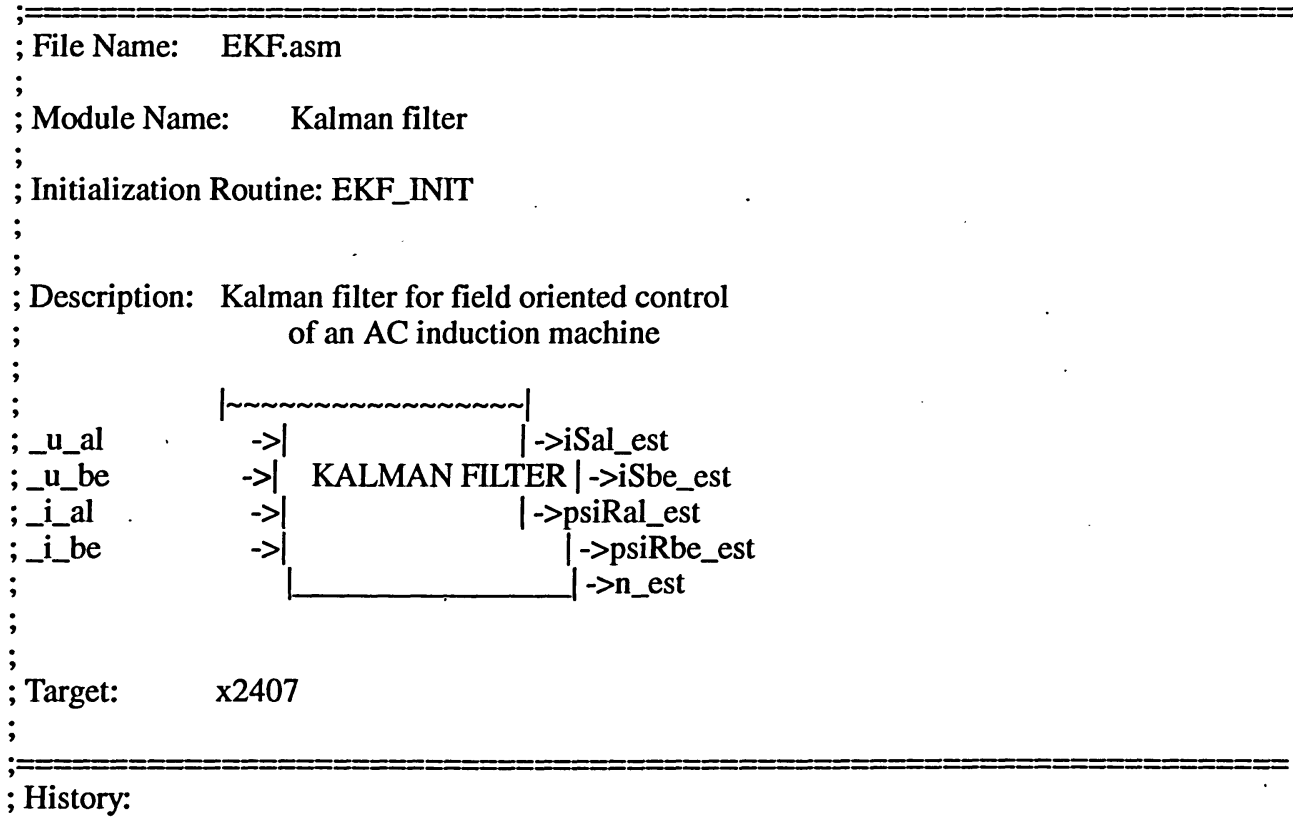




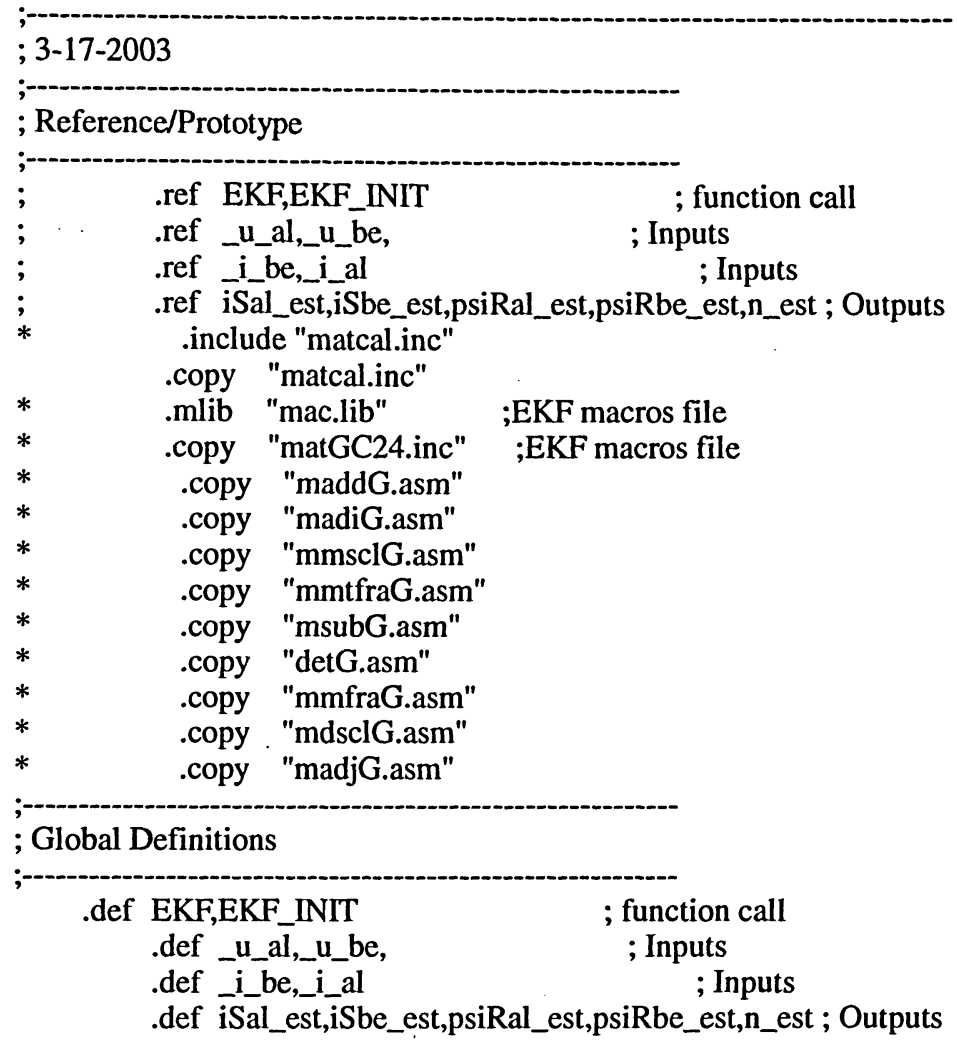

\section{; Variable Definitions}

;public variables

$\begin{array}{lcc}\text {;_u_al } & \text {.usect "ekf",1 } & \\ \text {;_u_be } & \text {.usect "ekf",1 } & \\ \text {;_i_be } & \text {.usect "ekf",1 } & \\ \text {;_i_al } & \text {.usect "ekf",1 } & \\ \text { iSal_est } & \text {.usect "ekf",1 } & \text {;alfa-axis estimated current, x(1) } \\ \text { iSbe_est } & \text {.usect "ekf",1 } & \text {;beta-axis estimated current, x(2) } \\ \text { psiRal_est } & \text {.usect "ekf",1 } & \text {;alfa-axis estimated rotor flux, x(3) } \\ \text { psiRbe_est } & \text {.usect "ekf",1 } & \text {;beta-axis estimated rotor flux, } x(4) \\ \text { n_est } & \text {.usect "ekf",1 } & \text {;estimated electrical speed, } x(5)\end{array}$

;private variables

$\begin{array}{lll}\text { *psiR_est } & \text {.usect "ekf" ,1 } & \text {;rotor flux amplitude } \\ \text { *psiRal_est1 } & \text {.usect "ekf",1 } & \text {;1.15 format (alfa-axis estimated rotor flux), x(3) } \\ \text { *psiRbe_est1 } & \text {.usect "ekf",1 } & \text {;1.15 format (beta-axis estimated rotor flux), x(4) } \\ \text { *psiR_est1 } & \text {.usect "ekf",1 } & \text {;1.15 format rotor flux amplitude } \\ \text { *run } & \text {.usect "ekf",1 } & \text {;initialization flag } \\ \text { *est } & \text {.usect "ekf",1 } & \text {;estimation flag:0h=initialization, 1000h=sensorless }\end{array}$

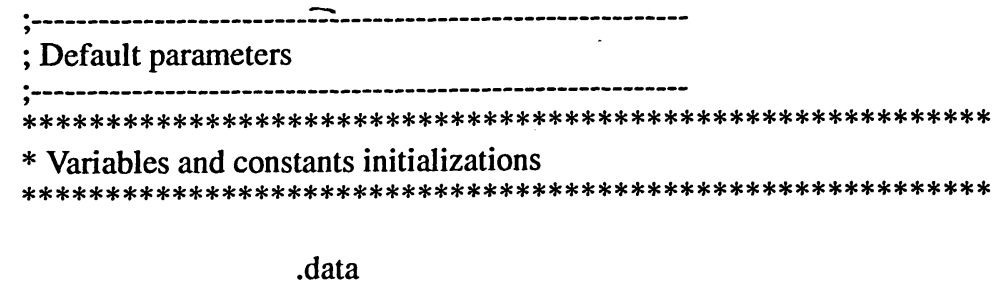


*** data page EKF algorithm ${ }^{* * *}$

kal_fil

;EKF algorithm data page pointer

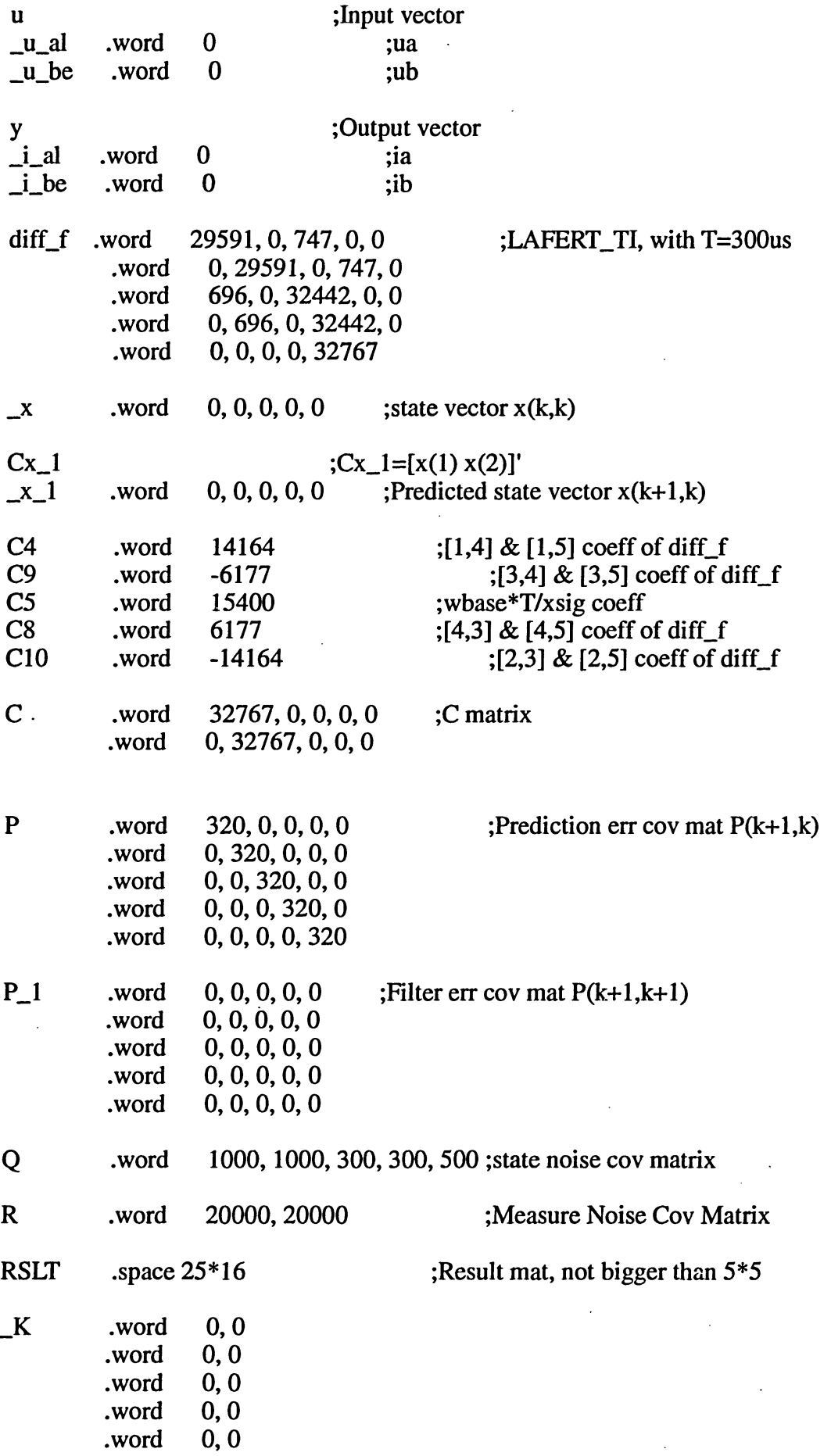

Pole_pairs_number .set 2

; Initialization 
.text

EKF_INIT

\begin{tabular}{|c|c|}
\hline \multicolumn{2}{|r|}{$\begin{array}{c}\text { \#n_est } \\
000 \mathrm{~h} \text {,iSal_est } \\
000 \text { h,iSbe_est }\end{array}$} \\
\hline $\begin{array}{l}\text { splk } \\
\text { splk }\end{array}$ & 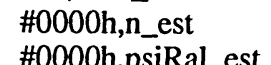 \\
\hline & $\# 0000 h$,psiRbe_est \\
\hline
\end{tabular}

EKF

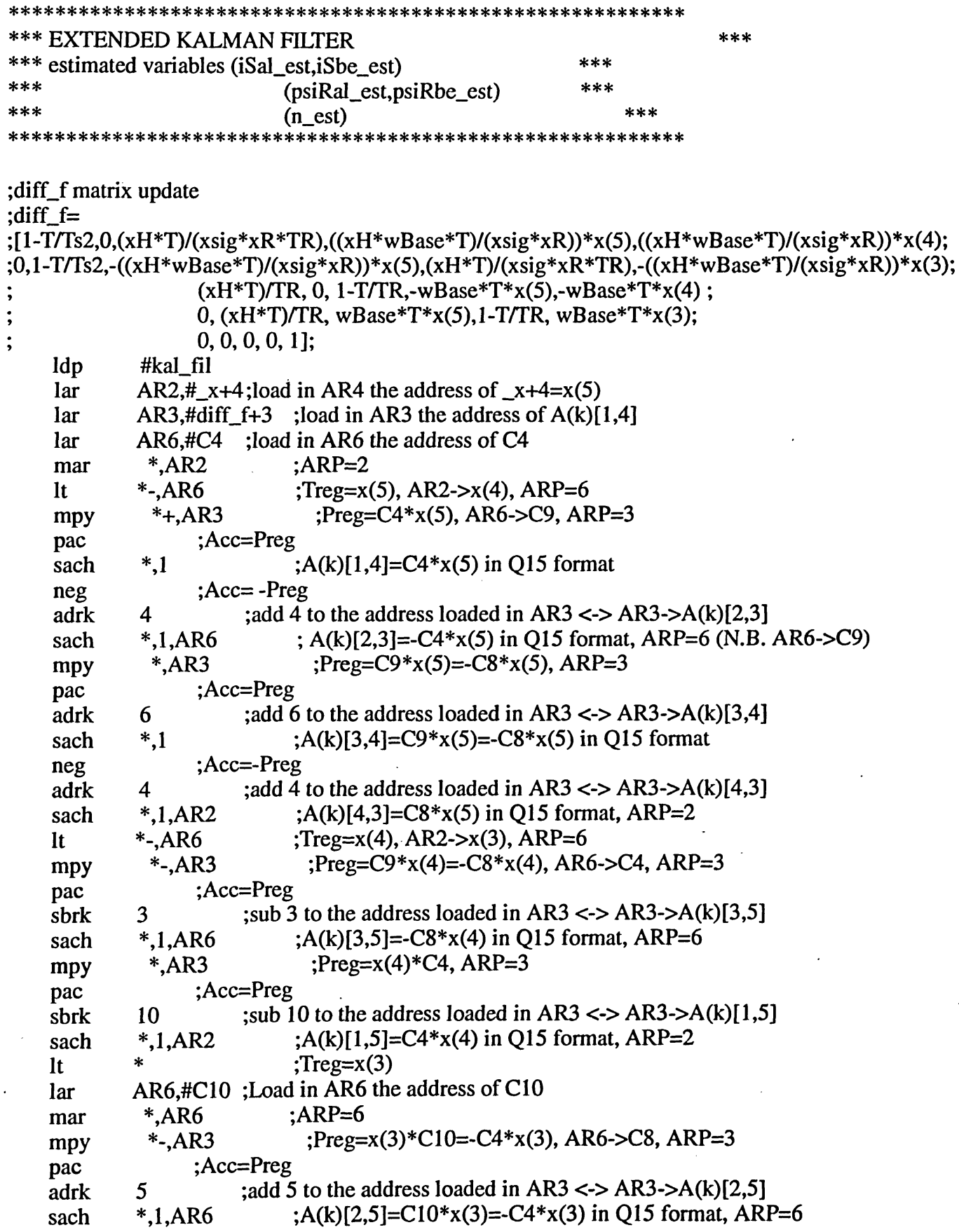




$$
\begin{array}{lll}
\text { mpy } & * \text { *AR3 } & \text {;Preg=x(3)*C8, ARP=3 } \\
\text { pac } & \multicolumn{2}{c}{; \text { Acc=Preg }} \\
\text { adrk } & 10 & \text {;add } 10 \text { to the address loaded in AR3 }<->\text { AR3->A(k)[4,5] } \\
\text { sach } & *, 1 & ; A(k)[4,5]=C 8 * x(3) \text { in } Q 15 \text { format. }
\end{array}
$$

;diff_f matrix is now updated

$$
\begin{aligned}
& \text {; } \mathrm{x} \_1=\text { state vector prediction }=\mathrm{x}(\mathrm{k} / \mathrm{k}-1) \\
& \text {; } \mathrm{x} \_1=[\text { diff_f }(1,1) * \mathrm{x}(1)+\text { diff_f }(1,3) * \mathrm{x}(3)+\text { diff_f }(1,4) * \mathrm{x}(4) \text {; } \\
& \text {; } \quad \text { diff_f }(2,2) * x(2)+\text { diff_f }(2,3) * x(3)+\text { diff_f }^{*}(2,4) * x(4) \text {; } \\
& \text { diff_f }(3,1) * x(1)+\text { diff_f }(3,3) * x(3)+\text { diff_f }(3,4) * x(4) \text {; } \\
& \text { diff_f }(4,2) * x(2)+\text { diff_f }(4,3) * x(3)+\text { diff_f }(4,4) * x(4) \text {; } \\
& \text { diff_f } f(5,5) * x(5)] . . . \\
& +[((w B a s e * T) / x s i g) * u(1) ;((w B a s e * T) / x \operatorname{sig}) * u(2) ; 0 ; 0 ; 0] \text {; }
\end{aligned}
$$

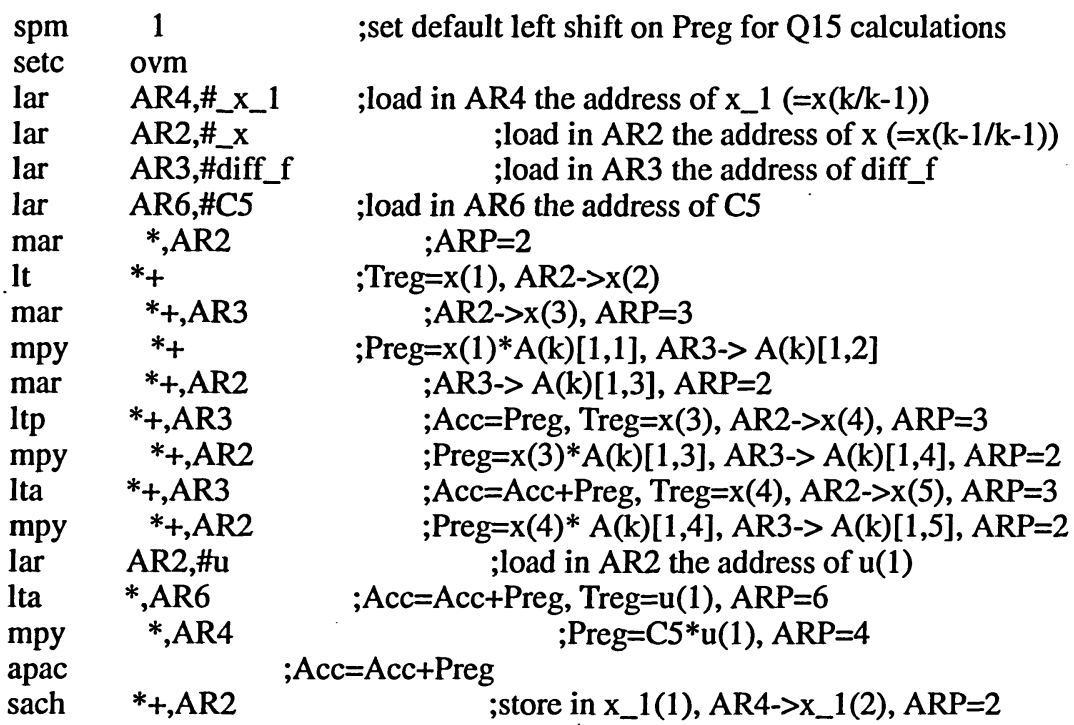

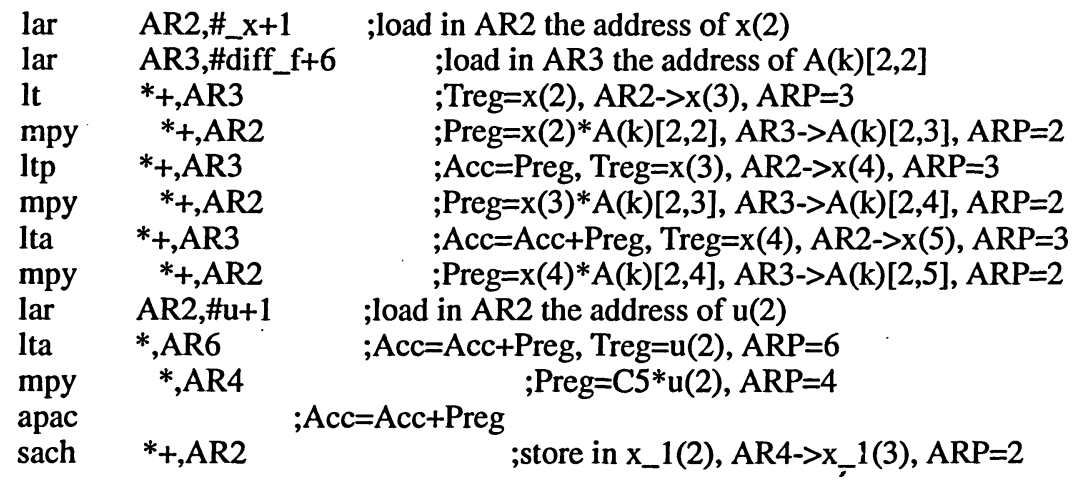

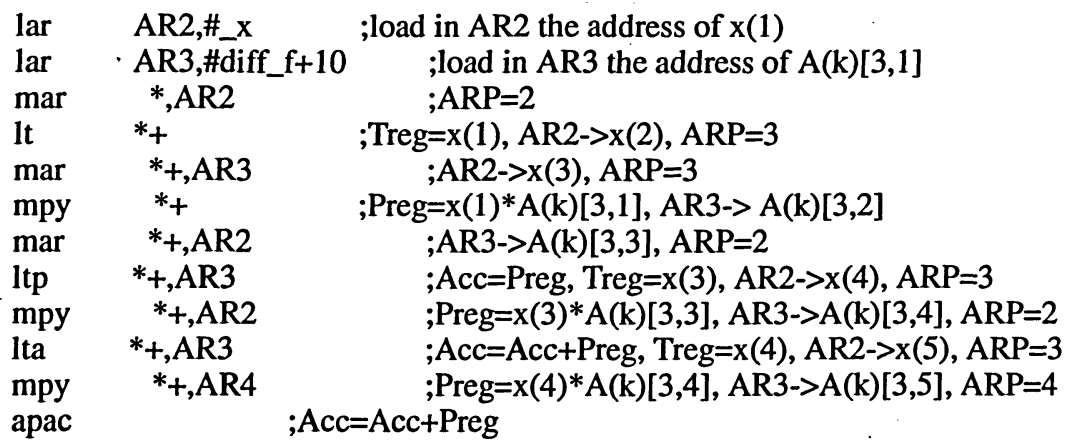



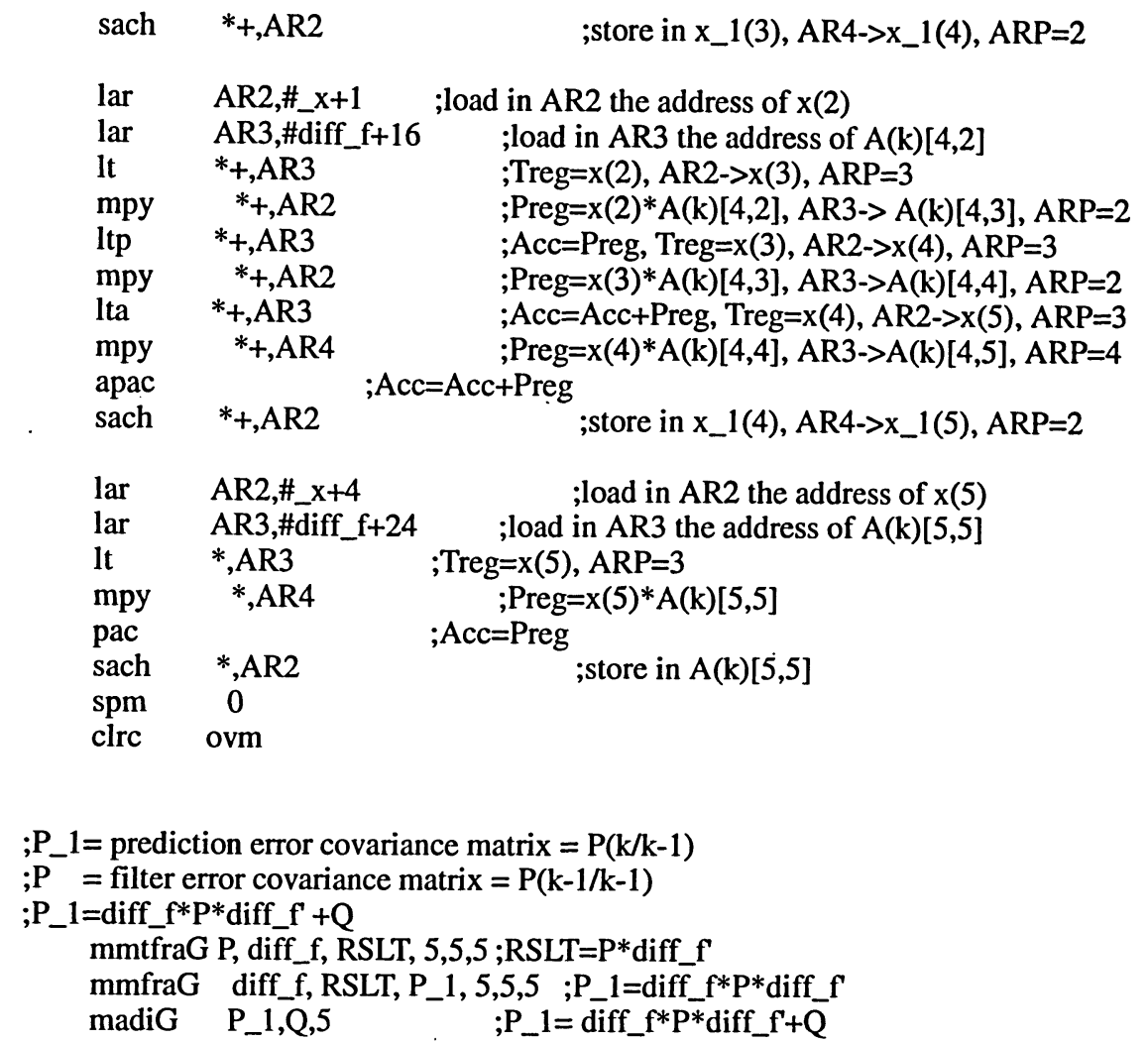

;Kalman gain matrix calculation

$; \mathrm{K}=\mathrm{P} \_1{ }^{*} \mathrm{C}^{\prime} * \operatorname{inv}\left(\mathrm{C}^{*} \mathrm{P} \_1 * \mathrm{C}^{\prime}+\mathrm{R}\right)$;

mmtfraG P_1, C, RSLT, 5,5,2

mmfraG C, RSLT, _K, 2,5,2

madiG _ $\mathrm{K}, \mathrm{R}, 2$;_K=_K+R

madjG_K, $\mathrm{P} \quad ; \mathrm{P}$ is a temp matrix, $\mathrm{P}=\operatorname{adj}(\mathrm{K})$

mmfraG RSLT, $P$, _K, 5, 2, 2 ;_K=RSLT*P=P_1*C'*adj(C*P_1*C'+R)

$\operatorname{detG} \quad \mathrm{P}, \mathrm{RSLT}-; \mathrm{RSLT}=\operatorname{det}(\mathrm{P})$

mdsclG_K, RSLT, _K, 5, 2, RSLT+1 ;_K=1/det(P)* P_1 ${ }^{*} C^{*} \operatorname{adj}\left(C^{*} \mathrm{P} \_1{ }^{*} \mathrm{C}^{\prime}+\mathrm{R}\right)$

;state vector estimation

$; \mathrm{x} \_1=$ state vector prediction $=\mathrm{x}(\mathrm{k} / \mathrm{k}-1)$

$; \mathrm{x}=$ state vector estimation

$; \mathrm{x}=\mathrm{x} \_1+\mathrm{K}^{*}\left(\mathrm{y}-\mathrm{C}^{*} \mathrm{x} \_1\right)$

msubG y, Cx_1, P, 2,1

;P is a temp matrix, $P=y-C^{*} x \_1$

mmfraG _K, P, RSLT, 5,2,1

maddG $\quad \mathrm{x} \_1, \mathrm{RSLT}, \mathrm{x}_{\mathrm{X}}, 5,1$

$$
\text { ;RSLT }=\_K^{*} P=\_K^{*}\left(y-C^{*} x \_1\right)
$$

; $x=x_{-} 1+K^{*}\left(y-C^{*} x_{-} \_\right)$

;Filter error covariance matrix

;P=P_1-K*C*P_1;

mmfraG

_K, C, RSLT, 5, 2, 5

mmfraG

RSLT, P_1, P , 5, 5, 5

msubG

P_1,P,P, 5,5

;RSLT $=\_\mathrm{K} * \mathrm{C}$

$; \mathrm{P}=\_\mathrm{K} * \mathrm{C} * \mathrm{P} \_1$

;P=P_1-P

ldp \#kal_fil

lacc_ $\quad \mathrm{x}$

ldp \#iSal_est

* sfr

* sfr

sacl iSal_est, 1

ldp \#kal_fil

lace $\quad x+1$
;iSalfa estimated*2

;notice that_x (estimated state) is in 1.15 format 


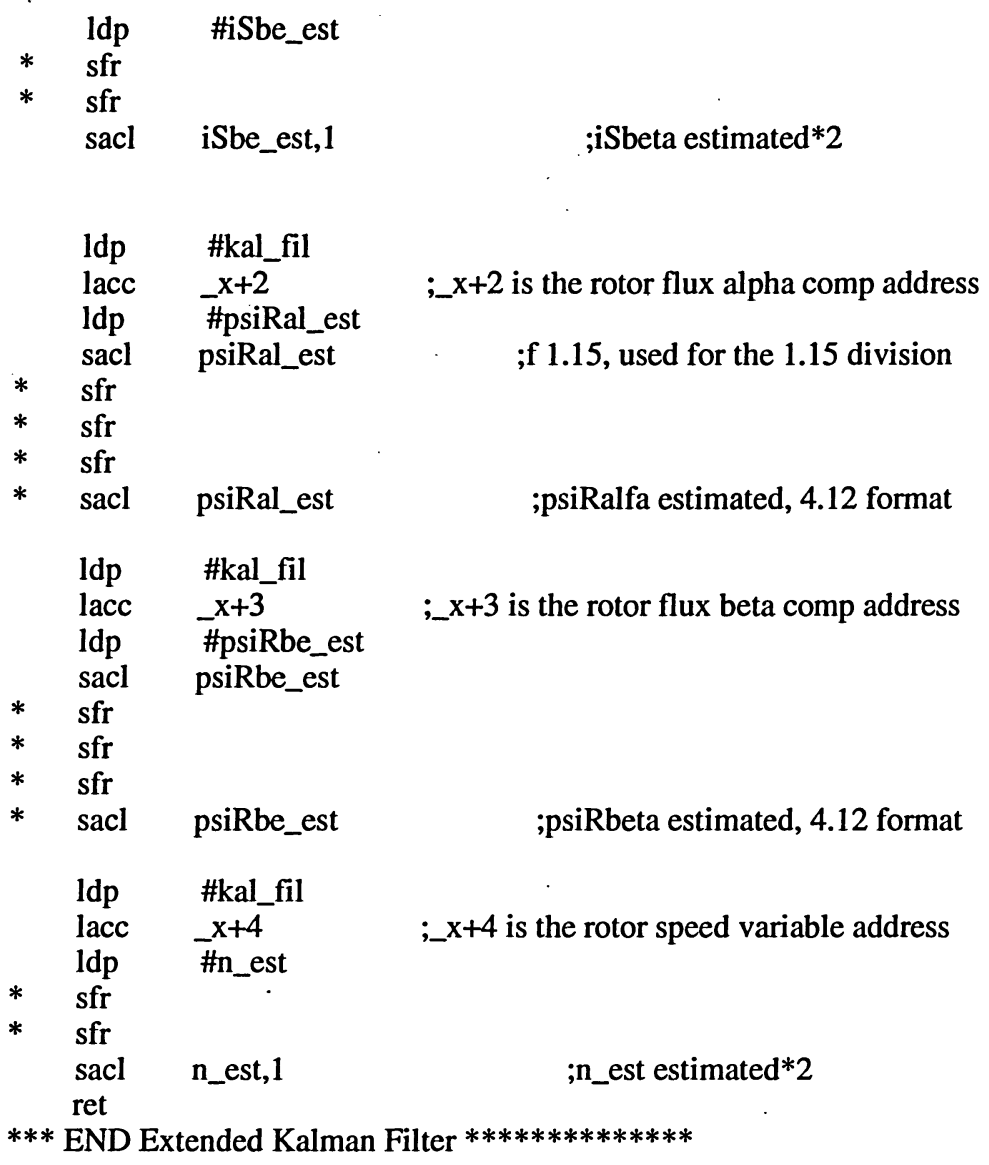

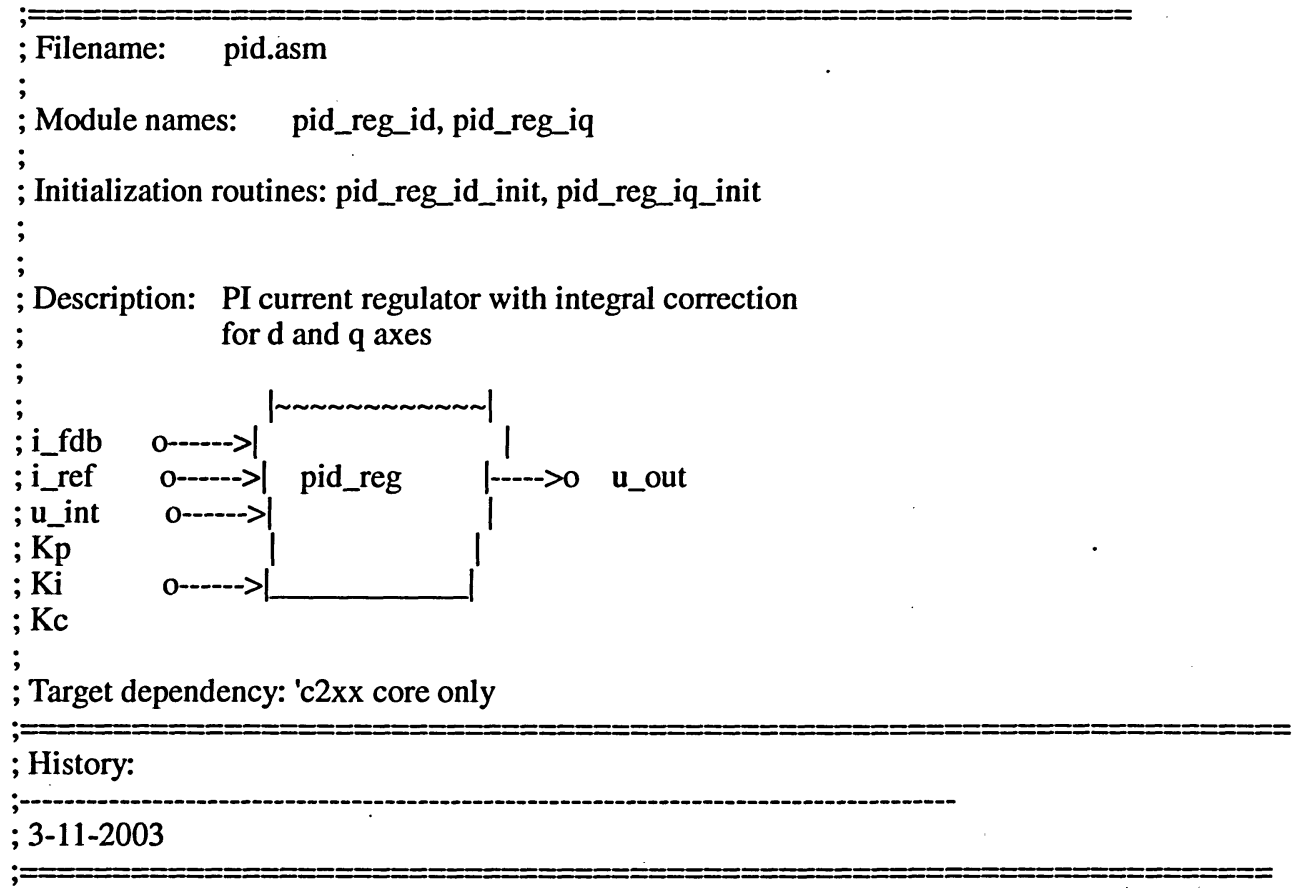




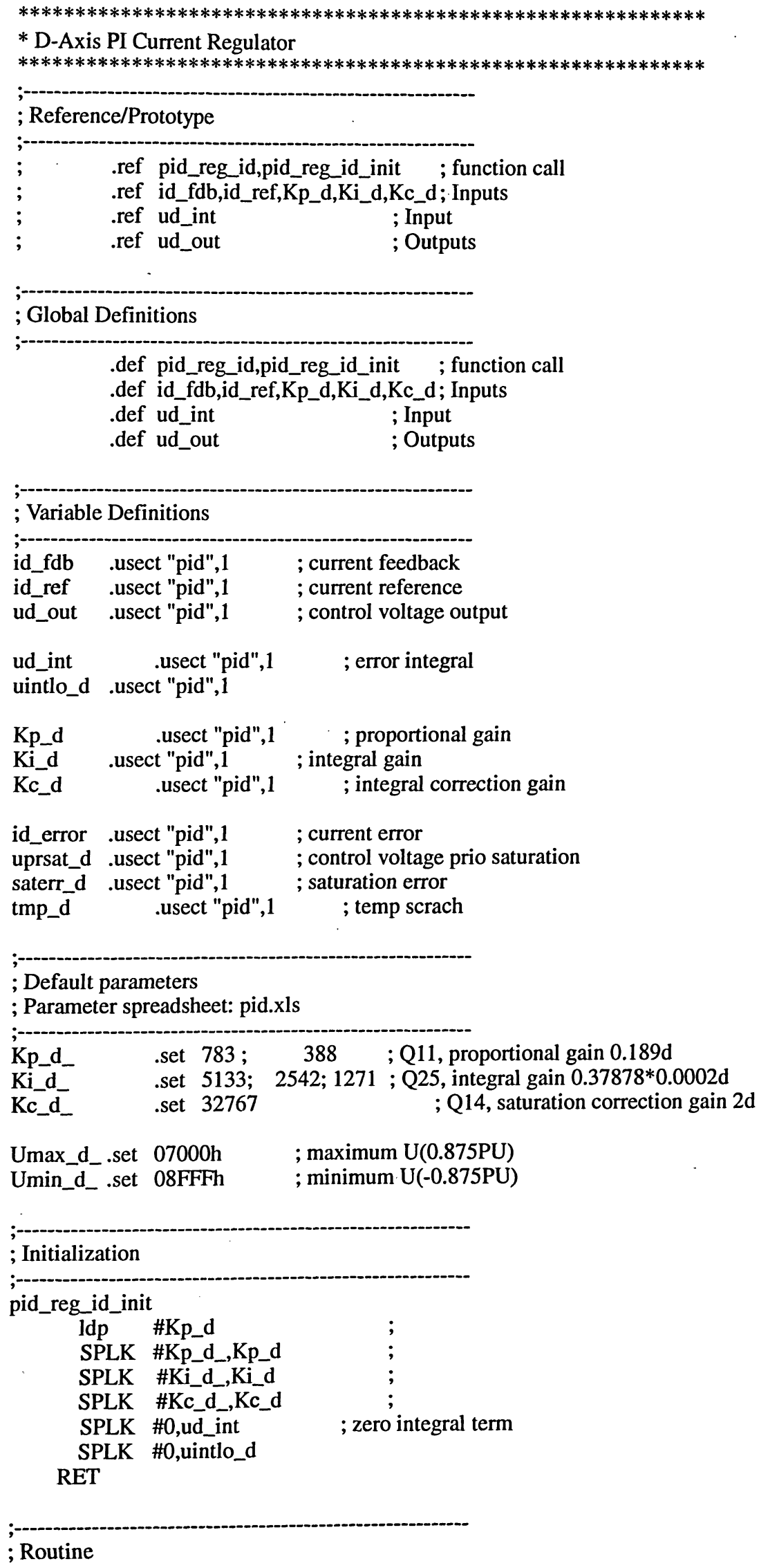




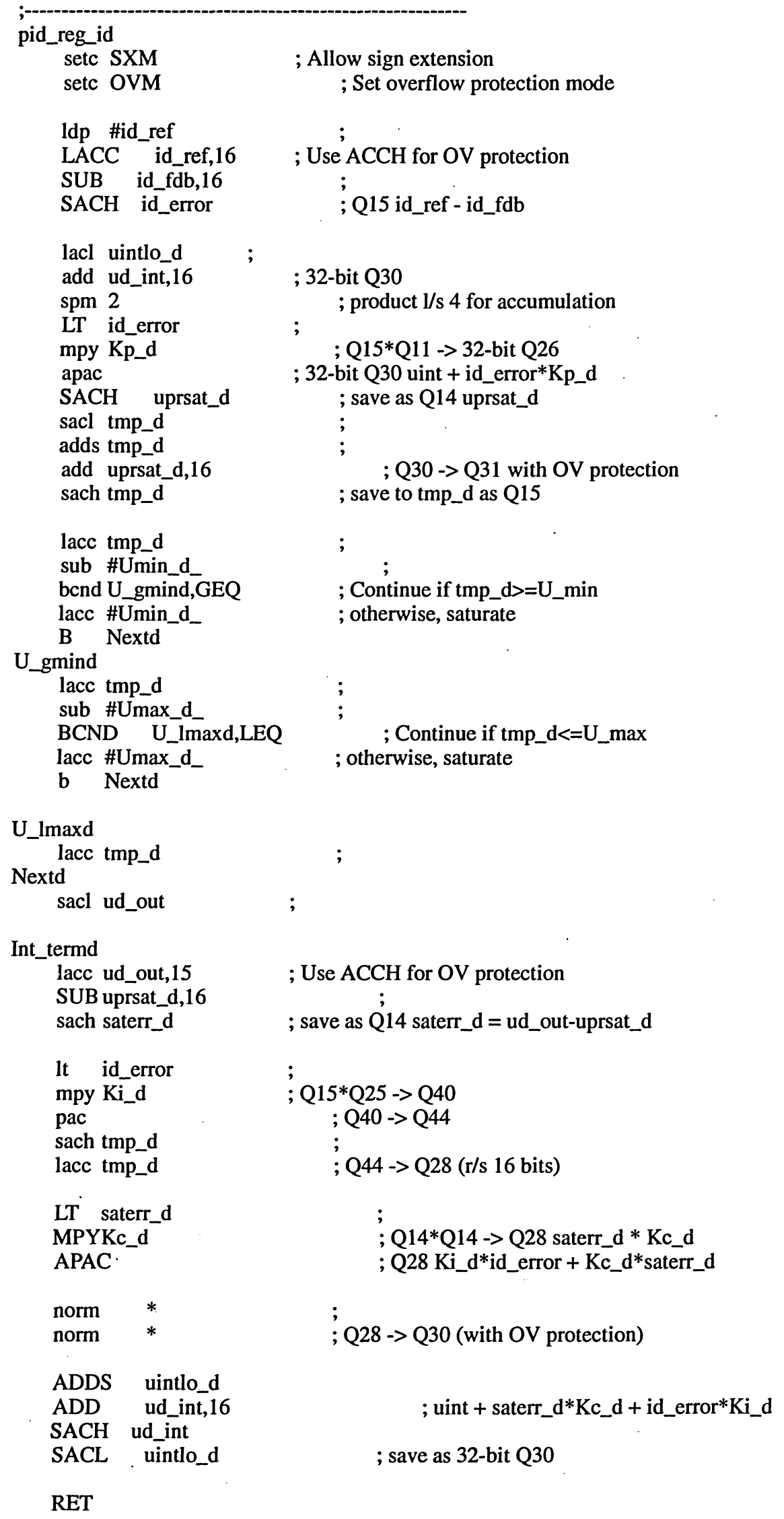




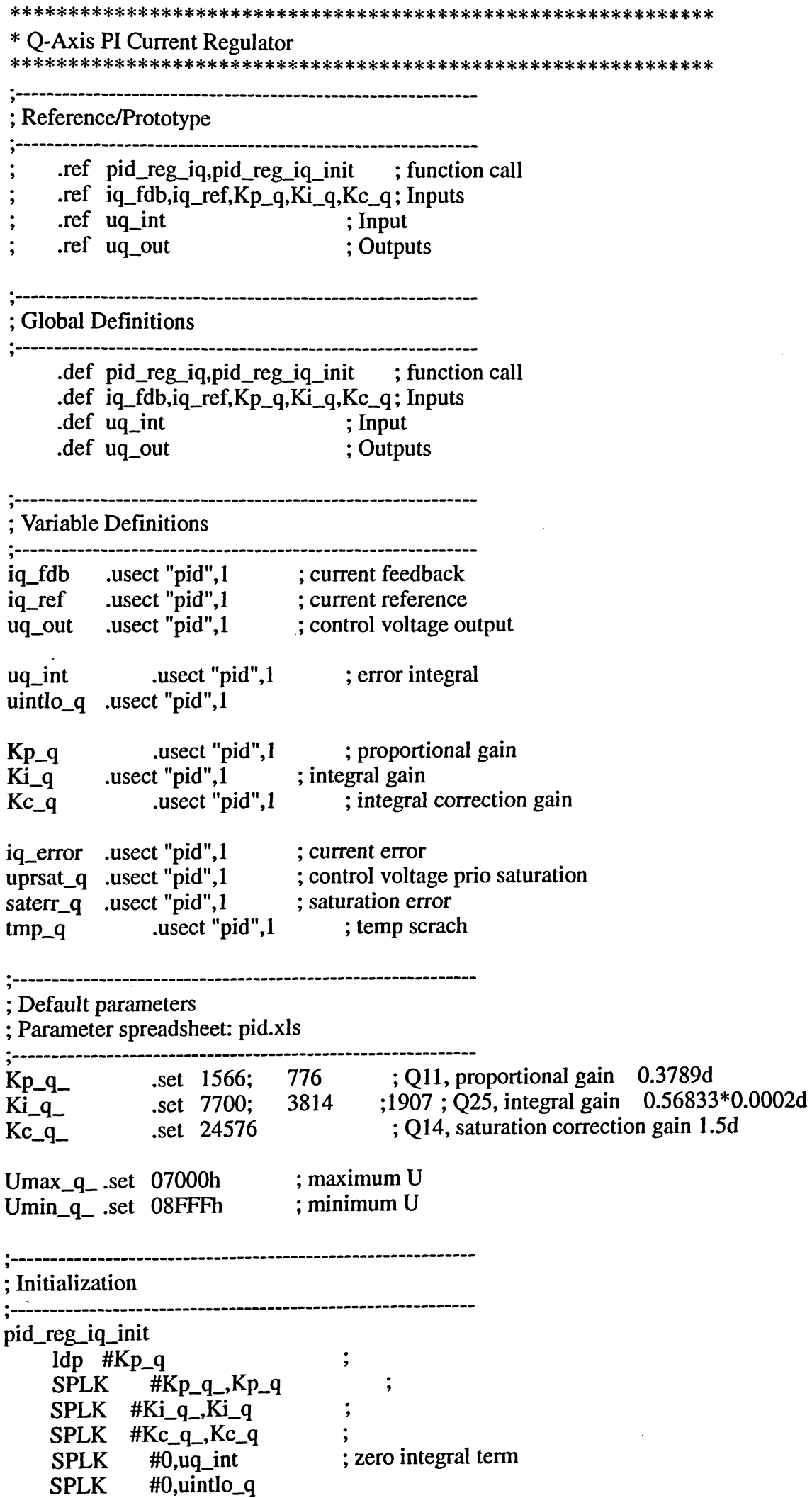


RET

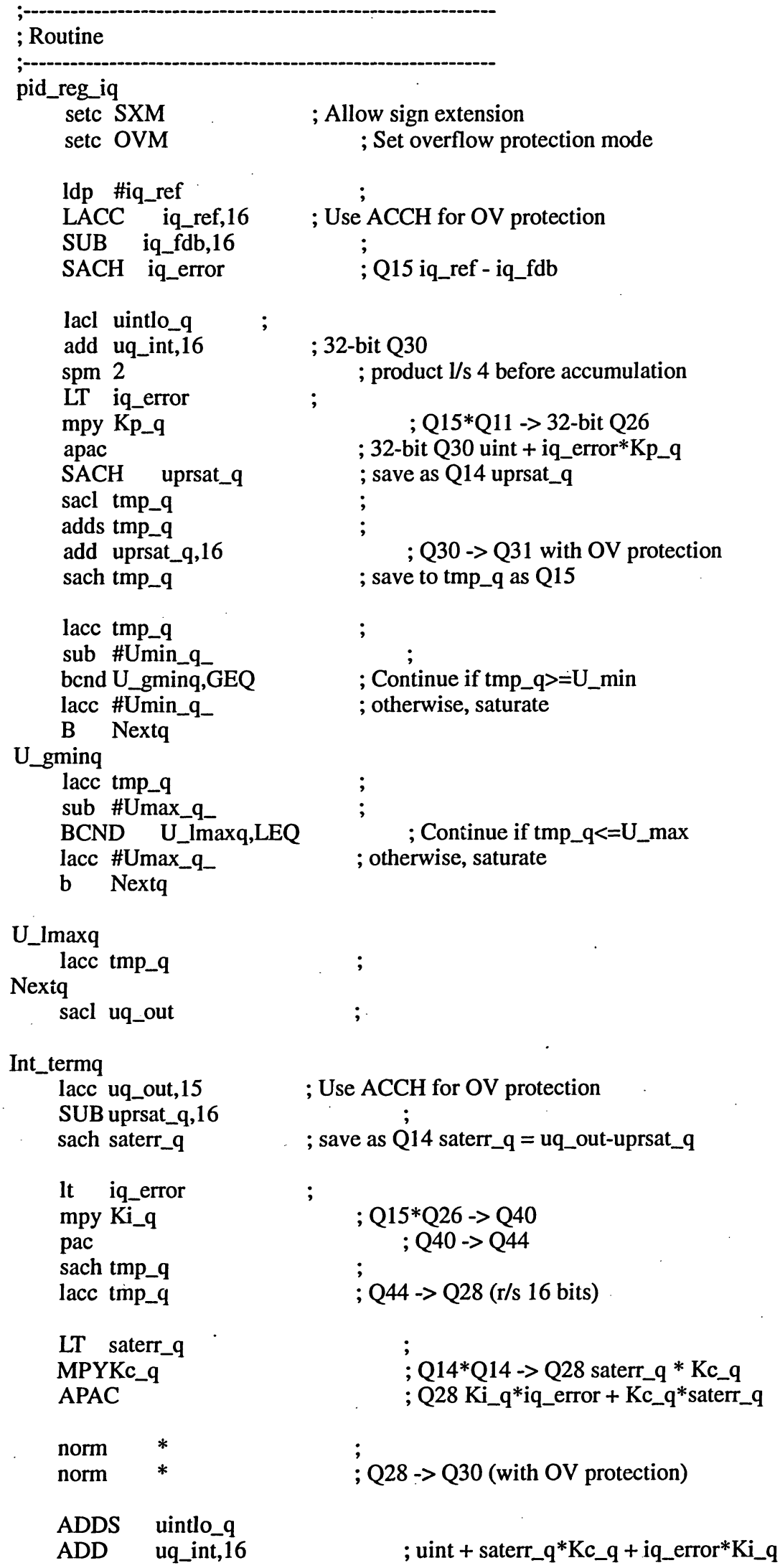


SACH

uq_int

SACL uintlo_q ; save as 32-bit Q30

RET

***END Q-Axis PI Current Regulator

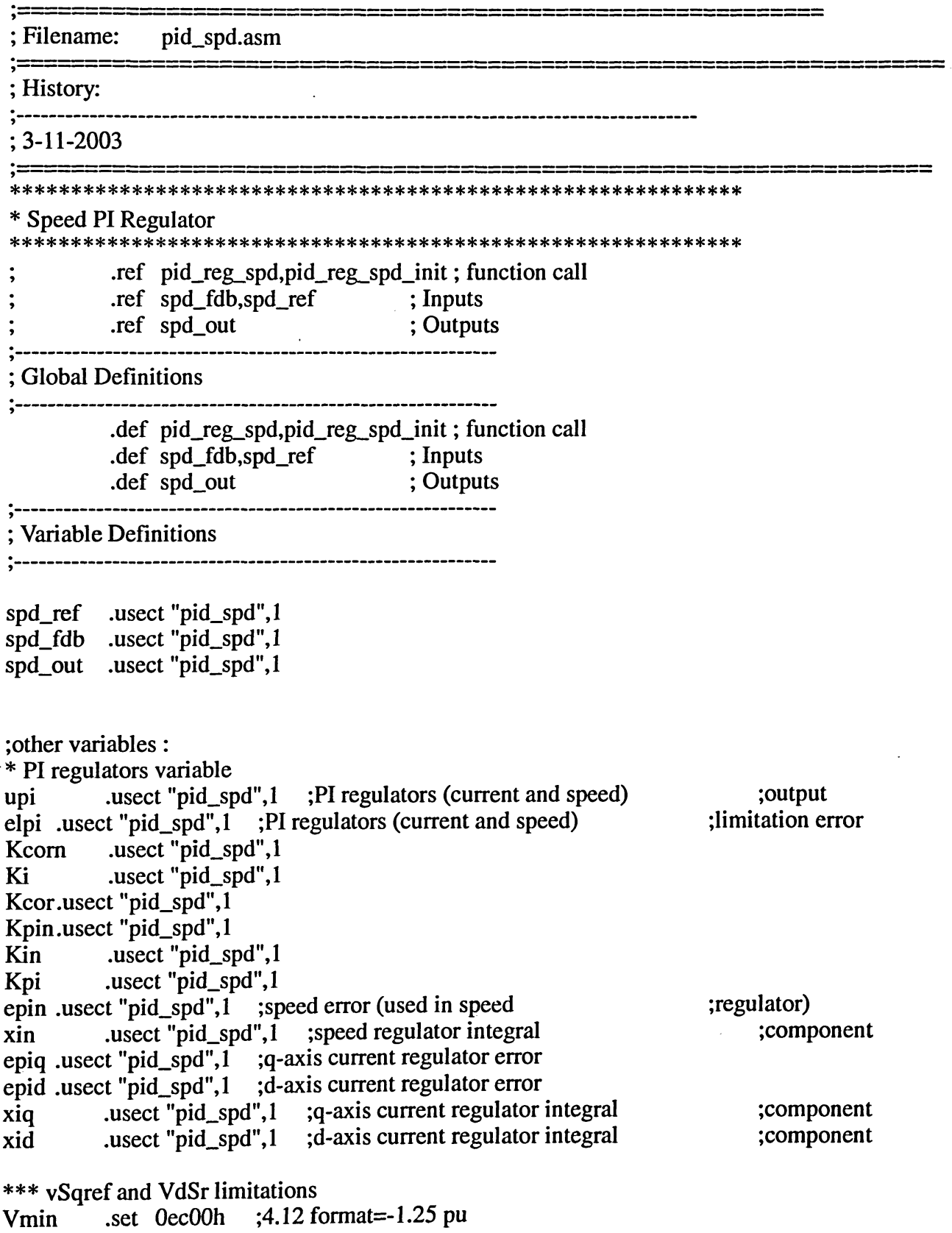




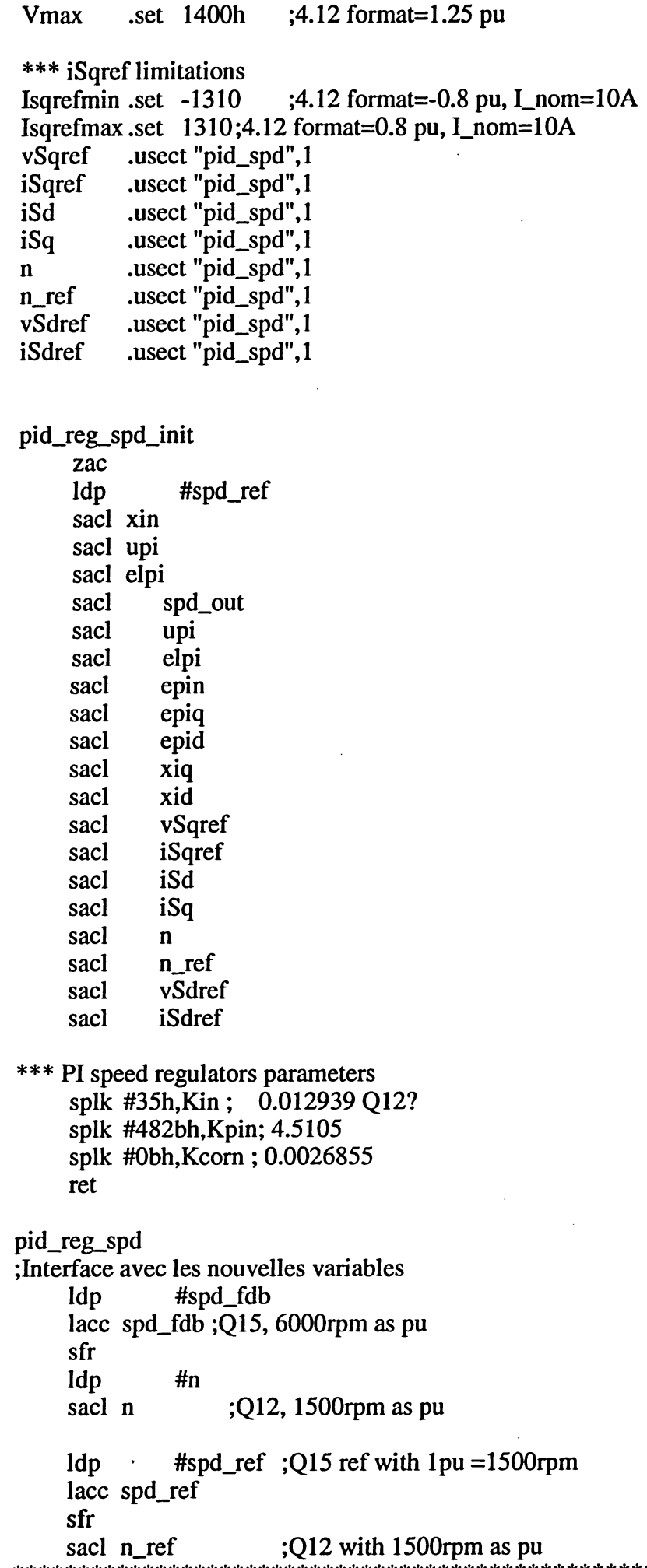


lacc xin, 12

It epin

mpy Kpin

apac

sach upi,4;upi=xin+epin*Kpin, 4.12 format

;here we start to saturate

bit upi,0

bcnd upimagzeros,NTC ;If value $>0$ we branch

lacc \#Isqrefmin ;negative saturation

sub upi

bcnd neg_sat,GT ;if upi<ISqrefmin then branch to ;saturate

lacc upi ;value of upi is valid

b limiters

neg_sat

lacc \#Isqrefmin ;set acc to -ve saturated value

b limiters

upimagzeros ;Value is positive

lacc \#Isqrefmax ;positive saturation

sub upi

bend pos_sat,LT ;if upi>ISqrefmax then branch to

lacc upi ; value of upi valid

;saturate

b limiters

pos_sat

lacc \#Isqrefmax ;set acc to +ve saturated value

limiters

sacl iSqref ;Store the acc as reference value

;Interface avec la nouvelle variable de sortie

setc OVM

sfl

sfl

sfl

ldp \#spd_out

sacl spd_out

sub \#7fffh

clrc OVM

ldp \#iSqref

lacc iSqref

sub upi

sacl elpi ;elpi=iSqref-upi, 4.12 format

lt elpi ;if there is no saturation elpi $=0$

mpy Kcorn

pac

lt epin

mpy Kin

apac

add xin, 12

sach xin,4;xin=xin+epin*Kin+elpi*Kcorn, 4.12

;format

$* * * * * * * * * * * * * * * * * * * * * * * * * * * * * * * * * * * * * * * * * * * * * * * * * * * * * * * * * * * *$

* END Speed regulator with integral component correction

$* * * * * * * * * * * * * * * * * * * * * * * * * * * * * * * * * * * * * * * * * * * * * * * * * * * * * * * * * * * *$

ret

; Filename: pwm_drv.asm

;

; Module Name: FC_PWM_DRV

; 

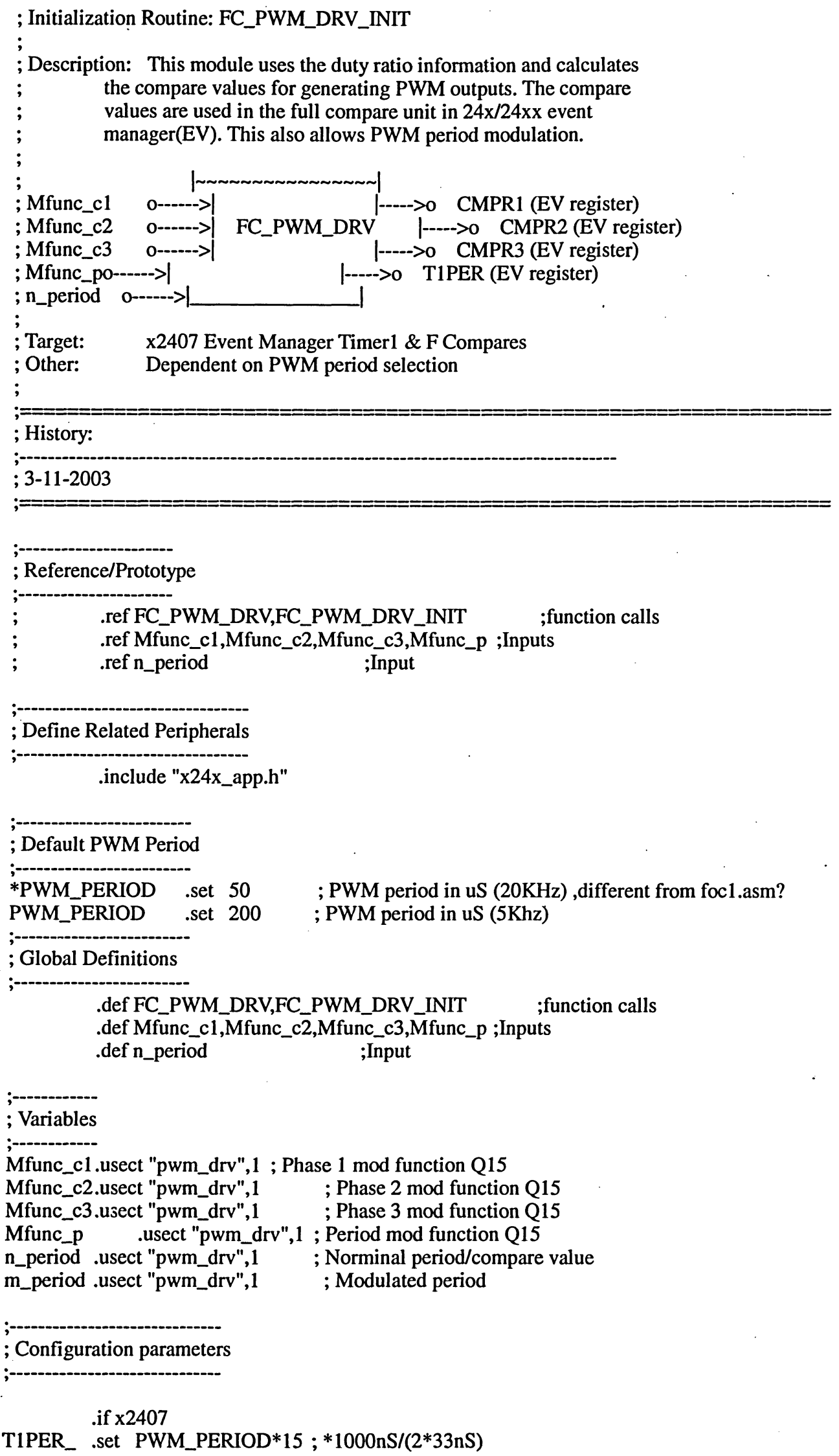


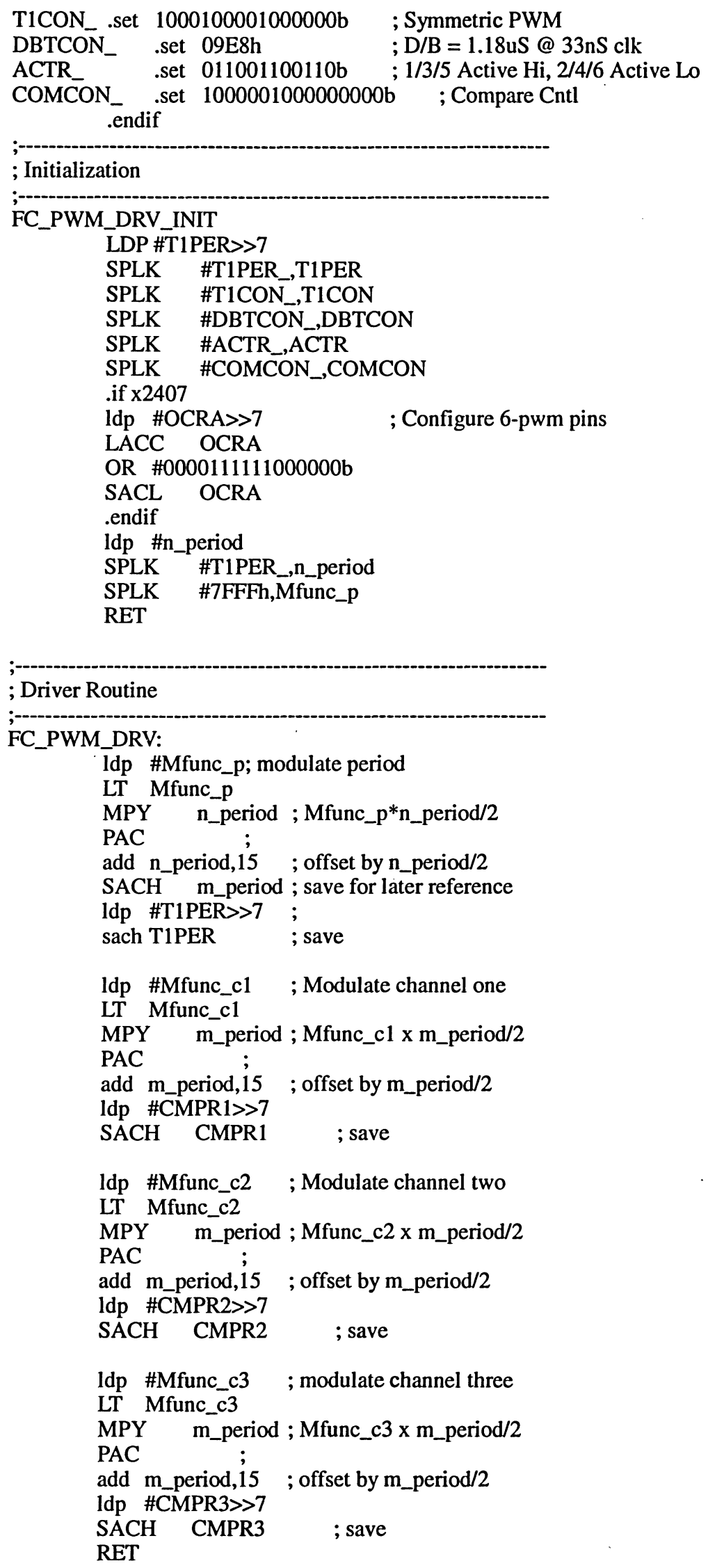




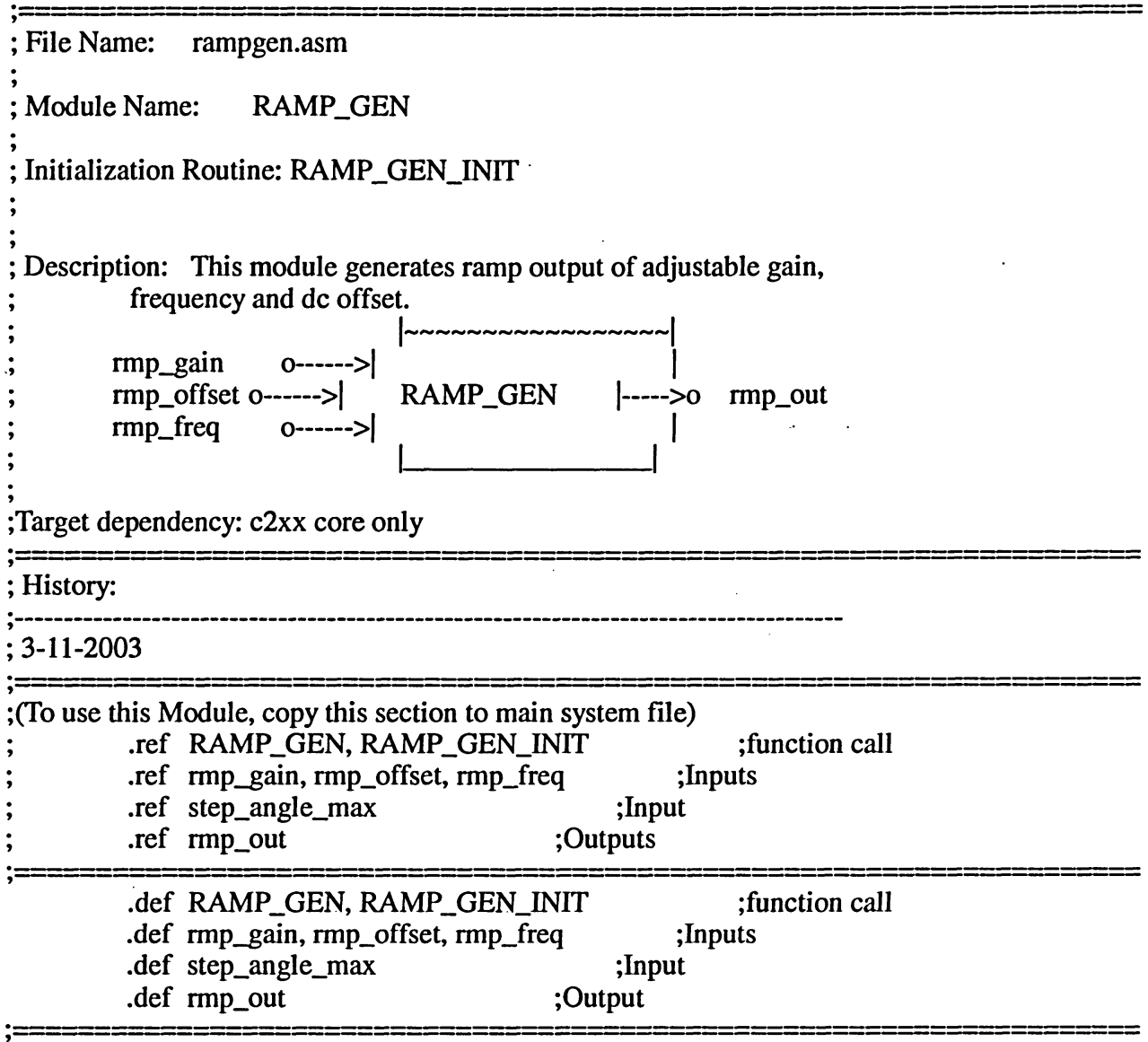

STEP_ANGLE_RG_MAX .set 1000 ;Corresponds to $305.2 \mathrm{~Hz}$ for $\mathrm{fs}=20 \mathrm{kHz}$ ; or $152.6 \mathrm{~Hz}$ for $\mathrm{fs}=10 \mathrm{kHz}$, or $76.3 \mathrm{~Hz}$ for $\mathrm{fs}=5 \mathrm{KHz}$ ;See related doc for details

alpha_rg .usect "rampgen",1

step_angle_rg .usect "rampgen",1

step_angle_max .usect "rampgen",1

rmp_gain .usect "rampgen",1

rmp_offset .usect "rampgen",1

rmp_freq .usect "rampgen",1

rmp_out .usect "rampgen",1

rmp_out_abs .usect "rampgen",1

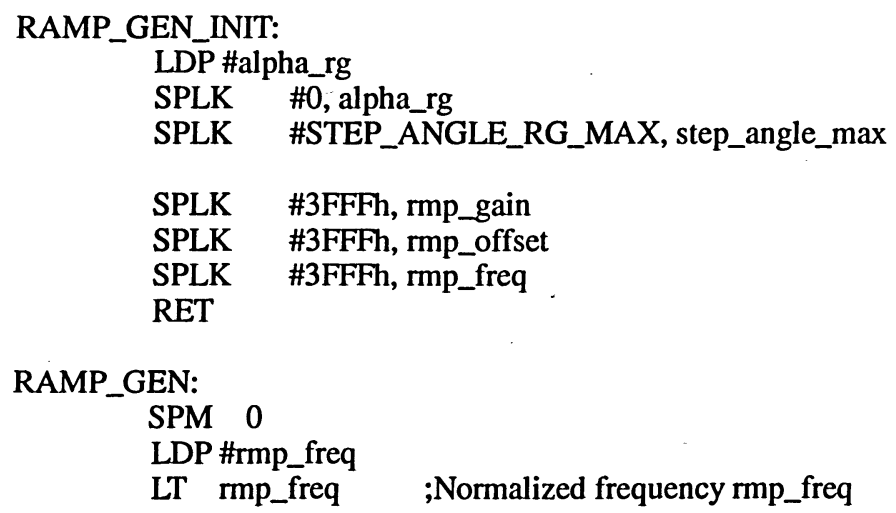




\begin{tabular}{llc} 
& \multicolumn{2}{c}{;is in $\mathrm{Q} 15$} \\
MPY & step_angle_max & ;Q15 x Q0 \\
PAC & \multicolumn{2}{c}{$; \mathrm{Q} 0 \times \mathrm{Q} 15=\mathrm{Q} 15(32 \mathrm{bit})$} \\
SACH & step_angle_rg,1 & $; \mathrm{Q} 0$ \\
& & \\
LACC & alpha_rg $; \mathrm{Q} 0$ \\
ADD & step_angle_rg & \\
SACL & alpha_rg $; \mathrm{Q} 0+\mathrm{Q} 0$
\end{tabular}

;scale the output with gain(user specified)

\begin{tabular}{lll} 
LT alpha_rg $; \mathrm{Q} 0$ \\
MPY & rmp_gain & $; \mathrm{Q} 0 \times \mathrm{Q} 15$ \\
PAC & \multicolumn{3}{c}{$; \mathrm{P}=$ rmp_gain * alpha_rg } \\
SACH & rmp_out_abs, 1 & $; \mathrm{Q} 0$ \\
LACC & rmp_out_abs & $; \mathrm{Q} 0$ \\
SACL & rmp_out & $; \mathrm{Q} 15^{* *}$
\end{tabular}

$* *$

;In the last two instructions the variables rmp_out_abs and rmp_out contain ; the same value which is the result of the preceeding multiply operation. ;Although they have the same value, by representing rmp_out with a ; different $\mathrm{Q}$ format $(\mathrm{Q} 15)$ than rmp_out_abs(Q0), we have essentially performed ; an implicit normalization(division) operation. The normalized ramp output, ;rmp_out(in Q15), and the absolute ramp output, rmp_out_abs (in Q0), are ;related by,

;rmp_out = rmp_out_abs/7FFFh.

;The output of this module(rmp_out) is normalized (expressed in Q15) since ;in many other $\mathrm{s} / \mathrm{w}$ modules, where this is used as input, require the input ;be provided in Q15 format.

; add offset value

$\begin{array}{lll}\text { LACC } & \text { rmp_out } & ; \mathrm{Q} 15 \\ \text { ADD } & \text { rmp_offset } & ; \mathrm{Q} 15+\mathrm{Q} 15 \\ \text { SACL } & \text { rmp_out } & ; \mathrm{Q} 15 \\ \text { RET } & & \end{array}$




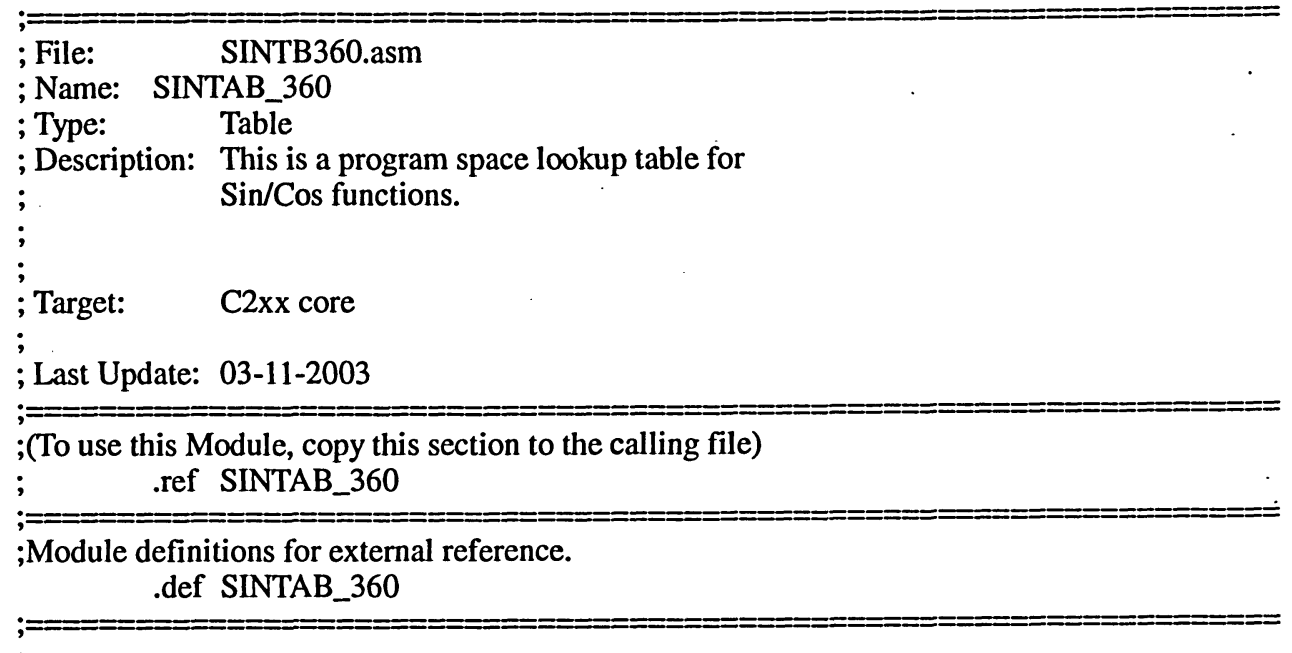

; Sine look-up table

; No. Entries: 256

; Angle Range: $360 \mathrm{deg}$

; Number format: $Q 15$ with range $-1<\mathrm{N}<+1$

\begin{tabular}{|c|c|c|c|c|c|c|}
\hline \multirow[b]{2}{*}{ SINTAB_360 } & \multicolumn{2}{|c|}{;SINVAL } & ; & Index & \multicolumn{2}{|c|}{ Angle Sin(Angle) } \\
\hline & .word & 0 & & 0 & 00 & \\
\hline & .word & 804 & ; & 1 & 1.41 & 0.0245 \\
\hline & .word & 1608 & ; & 2 & 2.81 & 0.0491 \\
\hline & .word & 2410 & ; & 3 & 4.22 & 0.0736 \\
\hline & .word & 3212 & ; & 4 & 5.63 & 0.0980 \\
\hline & .word & 4011 & ; & 5 & 7.03 & 0.1224 \\
\hline & .word & 4808 & ; & 6 & 8.44 & 0.1467 \\
\hline & .word & 5602 & ; & 7 & 9.84 & 0.1710 \\
\hline & .word & 6393 & ; & 8 & 11.25 & 0.1951 \\
\hline & .word & 7179 & ; & .9 & 12.66 & 0.2191 \\
\hline & .word & 7962 & ; & 10 & 14.06 & 0.2430 \\
\hline & .word & 8739 & ; & 11 & 15.47 & 0.2667 \\
\hline & .word & 9512 & ; & 12 & 16.88 & 0.2903 \\
\hline & .word & 10278 & ; & 13 & 18.28 & 0.3137 \\
\hline & .word & 11039 & ; & 14 & 19.69 & 0.3369 \\
\hline & .word & 11793 & ; & 15 & 21.09 & 0.3599 \\
\hline & .word & 12539 & ; & 16 & 22.50 & 0.3827 \\
\hline & .word & 13279 & ; & 17 & 23.91 & 0.4052 \\
\hline & .word & 14010 & ; & 18 & 25.31 & 0.4276 \\
\hline & .word & 14732 & ; & 19 & 26.72 & 0.4496 \\
\hline & .word & 15446 & ; & 20 & 28.13 & 0.4714 \\
\hline & .word & 16151 & ; & 21 & 29.53 & 0.4929 \\
\hline & .word & 16846 & ; & 22 & 30.94 & 0.5141 \\
\hline & .word & 17530 & ; & 23 & 32.34 & 0.5350 \\
\hline & .word & 18204 & ; & 24 & 33.75 & 0.5556 \\
\hline & .word & 18868 & ; & 25 & 35.16 & 0.5758 \\
\hline & .word & 19519 & ; & 26 & 36.56 & 0.5957 \\
\hline & .word & 20159 & ; & 27 & 37.97 & 0.6152 \\
\hline & .word & 20787 & ; & 28 & 39.38 & 0.6344 \\
\hline & .word & 21403 & ; & 29 & 40.78 & 0.6532 \\
\hline & .word & 22005 & ; & 30 & 42.19 & 0.6716 \\
\hline & .word & 22594 & ; & 31 & 43.59 & 0.6895 \\
\hline & .word & 23170 & ; & 32 & 45.00 & 0.7071 \\
\hline & .word & 23731 & ; & 33 & 46.41 & 0.7242 \\
\hline & .word & 24279 & ; & 34 & 47.81 & 0.7410 \\
\hline & .word & 24811 & ; & 35 & 49.22 & 0.7572 \\
\hline & .word & 25329 & ; & 36 & 50.63 & 0.7730 \\
\hline & .word & 25832 & ; & 37 & 52.03 & 0.7883 \\
\hline
\end{tabular}




\begin{tabular}{|c|c|c|c|c|c|}
\hline .word & 26319 & ; & 38 & 53.44 & 0.8032 \\
\hline .word & 26790 & ; & 39 & 54.84 & 0.8176 \\
\hline .word & 27245 & ; & 40 & 56.25 & 0.8315 \\
\hline .word & 27683 & ; & 41 & 57.66 & 0.8449 \\
\hline .word & 28105 & ; & 42 & 59.06 & 0.8577 \\
\hline .word & 28510 & ; & 43 & 60.47 & 0.8701 \\
\hline .word & 28898 & ; & 44 & 61.88 & 0.8819 \\
\hline .word & 29268 & ; & 45 & 63.28 & 0.8932 \\
\hline .word & 29621 & ; & 46 & 64.69 & 0.9040 \\
\hline .word & 29956 & ; & 47 & 66.09 & 0.9142 \\
\hline .word & 30273 & ; & 48 & 67.50 & 0.9239 \\
\hline .word & 30571 & ; & 49 & 68.91 & 0.9330 \\
\hline .word & 30852 & ; & 50 & 70.31 & 0.9415 \\
\hline .word & 31113 & ; & 51 & 71.72 & 0.9495 \\
\hline .word & 31356 & ; & 52 & 73.13 & 0.9569 \\
\hline .word & 31580 & ; & 53 & 74.53 & 0.9638 \\
\hline .word & 31785 & ; & 54 & 75.94 & 0.9700 \\
\hline .word & 31971 & ; & 55 & 77.34 & 0.9757 \\
\hline .word & 32137 & ; & 56 & 78.75 & 0.9808 \\
\hline .word & 32285 & ; & 57 & 80.16 & 0.9853 \\
\hline .word & 32412 & ; & 58 & 81.56 & 0.9892 \\
\hline .word & 32521 & ; & 59 & 82.97 & 0.9925 \\
\hline .word & 32609 & ; & 60 & 84.38 & 0.9952 \\
\hline .word & 32678 & ; & 61 & 85.78 & 0.9973 \\
\hline .word & 32728 & ; & 62 & 87.19 & 0.9988 \\
\hline .word & 32757 & ; & 63 & 88.59 & 0.9997 \\
\hline .word & 32767 & ; & 64 & 90.00 & 1.0000 \\
\hline .word & 32757 & ; & 65 & 91.41 & 0.9997 \\
\hline .word & 32728 & ; & 66 & 92.81 & 0.9988 \\
\hline .word & 32678 & ; & 67 & 94.22 & 0.9973 \\
\hline .word & 32609 & ; & 68 & 95.63 & 0.9952 \\
\hline .word & 32521 & ; & 69 & 97.03 & 0.9925 \\
\hline .word & 32412 & ; & 70 & 98.44 & 0.9892 \\
\hline .word & 32285 & ; & 71 & 99.84 & 0.9853 \\
\hline .word & 32137 & ; & 72 & 101.25 & 0.9808 \\
\hline .word & 31971 & ; & 73 & 102.66 & 0.9757 \\
\hline .word & 31785 & ; & 74 & 104.06 & 0.9700 \\
\hline .word & 31580 & ; & 75 & 105.47 & 0.9638 \\
\hline .word & 31356 & ; & 76 & 106.88 & 0.9569 \\
\hline .word & 31113 & ; & 77 & 108.28 & 0.9495 \\
\hline .word & 30852 & ; & 78 & 109.69 & 0.9415 \\
\hline .word & 30571 & ; & 79 & 111.09 & 0.9330 \\
\hline .word & 30273 & ; & 80 & 112.50 & 0.9239 \\
\hline .word & 29956 & ; & 81 & 113.91 & 0.9142 \\
\hline .word & 29621 & ; & 82 & 115.31 & 0.9040 \\
\hline .word & 29268 & ; & 83 & 116.72 & 0.8932 \\
\hline .word & 28898 & ; & 84 & 118.13 & 0.8819 \\
\hline .word & 28510 & ; & 85 & 119.53 & 0.8701 \\
\hline .word & 28105 & ; & 86 & 120.94 & 0.8577 \\
\hline .word & 27683 & ; & 87 & 122.34 & 0.8449 \\
\hline .word & 27245 & ; & 88 & 123.75 & 0.8315 \\
\hline .word & 26790 & ; & 89 & 125.16 & 0.8176 \\
\hline .word & 26319 & ; & 90 & 126.56 & 0.8032 \\
\hline .word & 25832 & ; & 91 & 127.97 & 0.7883 \\
\hline .word & 25329 & ; & 92 & 129.38 & 0.7730 \\
\hline .word & 24811 & ; & 93 & 130.78 & 0.7572 \\
\hline .word & 24279 & ; & 94 & 132.19 & 0.7410 \\
\hline .word & 23731 & ; & 95 & 133.59 & 0.7242 \\
\hline .word & 23170 & ; & 96 & 135.00 & 0.7071 \\
\hline .word & 22594 & ; & 97 & 136.41 & 0.6895 \\
\hline .word & 22005 & ; & 98 & 137.81 & 0.6716 \\
\hline .word & 21403 & ; & 99 & 139.22 & 0.6532 \\
\hline .word & 20787 & ; & 100 & 140.63 & 0.6344 \\
\hline
\end{tabular}




\begin{tabular}{|c|c|c|c|c|c|}
\hline .word & 20159 & ; & 101 & 142.03 & 0.6152 \\
\hline .word & 19519 & ; & 102 & 143.44 & 0.5957 \\
\hline .word & 18868 & ; & 103 & 144.84 & 0.5758 \\
\hline .word & 18204 & ; & 104 & 146.25 & 0.5556 \\
\hline .word & 17530 & ; & 105 & 147.66 & 0.5350 \\
\hline .word & 16846 & ; & 106 & 149.06 & 0.5141 \\
\hline .word & 16151 & ; & 107 & 150.47 & 0.4929 \\
\hline .word & 15446 & ; & 108 & 151.88 & 0.4714 \\
\hline .word & 14732 & ; & 109 & 153.28 & 0.4496 \\
\hline .word & 14010 & ; & 110 & 154.69 & 0.4276 \\
\hline .word & 13279 & ; & 111 & 156.09 & 0.4052 \\
\hline .word & 12539 & ; & 112 & 157.50 & 0.3827 \\
\hline .word & 11793 & ; & 113 & 158.91 & 0.3599 \\
\hline .word & 11039 & ; & 114 & 160.31 & 0.3369 \\
\hline .word & 10278 & ; & 115 & 161.72 & 0.3137 \\
\hline .word & 9512 & ; & 116 & 163.13 & 0.2903 \\
\hline .word & 8739 & ; & 117 & 164.53 & 0.2667 \\
\hline .word & 7962 & ; & 118 & 165.94 & 0.2430 \\
\hline .word & 7179 & ; & 119 & 167.34 & 0.2191 \\
\hline .word & 6393 & ; & 120 & 168.75 & 0.1951 \\
\hline .word & 5602 & ; & 121 & 170.16 & 0.1710 \\
\hline .word & 4808 & ; & 122 & 171.56 & 0.1467 \\
\hline .word & 4011 & ; & 123 & 172.97 & 0.1224 \\
\hline .word & 3212 & ; & 124 & 174.38 & 0.0980 \\
\hline .word & 2410 & ; & 125 & 175.78 & 0.0736 \\
\hline .word & 1608 & ; & 126 & 177.19 & 0.0491 \\
\hline .word & 804 & ; & 127 & 178.59 & 0.0245 \\
\hline .word & 0 & ; & 128 & 180.00 & 0.0000 \\
\hline .word & 64731 & ; & 129 & 181.41 & -0.0245 \\
\hline .word & 63927 & ; & 130 & 182.81 & -0.0491 \\
\hline .word & 63125 & ; & 131 & 184.22 & -0.0736 \\
\hline .word & 62323 & ; & 132 & 185.63 & -0.0980 \\
\hline .word & 61524 & ; & 133 & 187.03 & -0.1224 \\
\hline .word & 60727 & ; & 134 & 188.44 & -0.1467 \\
\hline .word & 59933 & ; & 135 & 189.84 & -0.1710 \\
\hline .word & 59142 & ; & 136 & 191.25 & -0.1951 \\
\hline .word & 58356 & ; & 137 & 192.66 & -0.2191 \\
\hline .word & 57573 & ; & 138 & 194.06 & -0.2430 \\
\hline .word & 56796 & ; & 139 & 195.47 & -0.2667 \\
\hline .word & 56023 & ; & 140 & 196.88 & -0.2903 \\
\hline .word & 55257 & ; & 141 & 198.28 & -0.3137 \\
\hline .word & 54496 & ; & 142 & 199.69 & -0.3369 \\
\hline .word & 53742 & ; & 143 & 201.09 & -0.3599 \\
\hline .word & 52996 & ; & 144 & 202.50 & -0.3827 \\
\hline .word & 52256 & ; & 145 & 203.91 & -0.4052 \\
\hline .word & 51525 & ; & 146 & 205.31 & -0.4276 \\
\hline .word & 50803 & ; & 147 & 206.72 & -0.4496 \\
\hline .word & 50089 & ; & 148 & 208.13 & -0.4714 \\
\hline .word & 49384 & ; & 149 & 209.53 & -0.4929 \\
\hline .word & 48689 & ; & 150 & 210.94 & -0.5141 \\
\hline .word & 48005 & ; & 151 & 212.34 & -0.5350 \\
\hline .word & 47331 & ; & 152 & 213.75 & -0.5556 \\
\hline .word & 46667 & ; & 153 & 215.16 & -0.5758 \\
\hline .word & 46016 & ; & 154 & 216.56 & -0.5957 \\
\hline .word & 45376 & ; & 155 & 217.97 & -0.6152 \\
\hline .word & 44748 & ; & 156 & 219.38 & -0.6344 \\
\hline .word & 44132 & ; & 157 & 220.78 & -0.6532 \\
\hline .word & 43530 & ; & 158 & 222.19 & -0.6716 \\
\hline .word & 42941 & ; & 159 & 223.59 & -0.6895 \\
\hline .word & 42365 & ; & 160 & 225.00 & -0.7071 \\
\hline .word & 41804 & ; & 161 & 226.41 & -0.7242 \\
\hline .word & 41256 & ; & 162 & 227.81 & -0.7410 \\
\hline .word & 40724 & ; & 163 & 229.22 & -0.7572 \\
\hline
\end{tabular}




\begin{tabular}{|c|c|c|c|c|c|}
\hline .word & 40206 & ; & 164 & 230.63 & -0.7730 \\
\hline .word & 39703 & ; & 165 & 232.03 & -0.7883 \\
\hline .word & 39216 & ; & 166 & 233.44 & -0.8032 \\
\hline .word & 38745 & ; & 167 & 234.84 & -0.8176 \\
\hline .word & 38290 & ; & 168 & 236.25 & -0.8315 \\
\hline .word & 37852 & ; & 169 & 237.66 & -0.8449 \\
\hline .word & 37430 & ; & 170 & 239.06 & -0.8577 \\
\hline .word & 37025 & ; & 171 & 240.47 & -0.8701 \\
\hline .word & 36637 & ; & 172 & 241.88 & -0.8819 \\
\hline .word & 36267 & ; & 173 & 243.28 & -0.8932 \\
\hline .word & 35914 & ; & 174 & 244.69 & -0.9040 \\
\hline .word & 35579 & ; & 175 & 246.09 & -0.9142 \\
\hline .word & 35262 & ; & 176 & 247.50 & -0.9239 \\
\hline .word & 34964 & ; & 177 & 248.91 & -0.9330 \\
\hline .word & 34683 & ; & 178 & 250.31 & -0.9415 \\
\hline .word & 34422 & ; & 179 & 251.72 & -0.9495 \\
\hline .word & 34179 & ; & 180 & 253.13 & -0.9569 \\
\hline .word & 33955 & ; & 181 & 254.53 & -0.9638 \\
\hline .word & 33750 & ; & 182 & 255.94 & -0.9700 \\
\hline .word & 33564 & ; & 183 & 257.34 & -0.9757 \\
\hline .word & 33398 & ; & 184 & 258.75 & -0.9808 \\
\hline .word & 33250 & ; & 185 & 260.16 & -0.9853 \\
\hline .word & 33123 & ; & 186 & 261.56 & -0.9892 \\
\hline .word & 33014 & ; & 187 & 262.97 & -0.9925 \\
\hline .word & 32926 & ; & 188 & 264.38 & -0.9952 \\
\hline .word & 32857 & ; & 189 & 265.78 & -0.9973 \\
\hline .word & 32807 & ; & 190 & 267.19 & -0.9988 \\
\hline .word & 32778 & ; & 191 & 268.59 & -0.9997 \\
\hline .word & 32768 & ; & 192 & 270.00 & -1.0000 \\
\hline .word & 32778 & ; & 193 & 271.41 & -0.9997 \\
\hline .word & 32807 & ; & 194 & 272.81 & -0.9988 \\
\hline .word & 32857 & ; & 195 & 274.22 & -0.9973 \\
\hline .word & 32926 & ; & 196 & 275.63 & -0.9952 \\
\hline .word & 33014 & ; & 197 & 277.03 & -0.9925 \\
\hline .word & 33123 & ; & 198 & 278.44 & -0.9892 \\
\hline .word & 33250 & ; & 199 & 279.84 & -0.9853 \\
\hline .word & 33398 & ; & 200 & 281.25 & -0.9808 \\
\hline .word & 33564 & ; & 201 & 282.66 & -0.9757 \\
\hline .word & 33750 & ; & 202 & 284.06 & -0.9700 \\
\hline .word & 33955 & ; & 203 & 285.47 & -0.9638 \\
\hline .word & 34179 & ; & 204 & 286.88 & -0.9569 \\
\hline .word & 34422 & ; & 205 & 288.28 & -0.9495 \\
\hline .word & 34683 & ; & 206 & 289.69 & -0.9415 \\
\hline .word & 34964 & ; & 207 & 291.09 & -0.9330 \\
\hline .word & 35262 & ; & 208 & 292.50 & -0.9239 \\
\hline .word & 35579 & ; & 209 & 293.91 & -0.9142 \\
\hline .word & 35914 & ; & 210 & 295.31 & -0.9040 \\
\hline .word & 36267 & ; & 211 & 296.72 & -0.8932 \\
\hline .word & 36637 & ; & 212 & 298.13 & -0.8819 \\
\hline .word & 37025 & ; & 213 & 299.53 & -0.8701 \\
\hline .word & 37430 & ; & 214 & 300.94 & -0.8577 \\
\hline .word & 37852 & ; & 215 & 302.34 & -0.8449 \\
\hline .word & 38290 & ; & 216 & 303.75 & -0.8315 \\
\hline .word & 38745 & ; & 217 & 305.16 & -0.8176 \\
\hline .word & 39216 & ; & 218 & 306.56 & -0.8032 \\
\hline .word & 39703 & ; & 219 & 307.97 & -0.7883 \\
\hline .word & 40206 & ; & 220 & 309.38 & -0.7730 \\
\hline .word & 40724 & ; & 221 & 310.78 & -0.7572 \\
\hline .word & 41256 & ; & 222 & 312.19 & -0.7410 \\
\hline .word & 41804 & ; & 223 & 313.59 & -0.7242 \\
\hline .word & 42365 & ; & 224 & 315.00 & -0.7071 \\
\hline .word & 42941 & ; & 225 & 316.41 & -0.6895 \\
\hline .word & 43530 & ; & 226 & 317.81 & -0.6716 \\
\hline
\end{tabular}




$\begin{array}{llllll}\text {.word } & 44132 & ; & 227 & 319.22 & -0.6532 \\ \text {.word } & 44748 & ; & 228 & 320.63 & -0.6344 \\ \text {.word } & 45376 & ; & 229 & 322.03 & -0.6152 \\ \text {.word } & 46016 & ; & 230 & 323.44 & -0.5957 \\ \text {.word } & 46667 & ; & 231 & 324.84 & -0.5758 \\ \text {.word } & 47331 & ; & 232 & 326.25 & -0.5556 \\ \text {.word } & 48005 & ; & 233 & 327.66 & -0.5350 \\ \text {.word } & 48689 & ; & 234 & 329.06 & -0.5141 \\ \text {.word } & 49384 & ; & 235 & 330.47 & -0.4929 \\ \text {.word } & 50089 & ; & 236 & .331 .88 & -0.4714 \\ \text {.word } & 50803 & ; & 237 & 333.28 & -0.4496 \\ \text {.word } & 51525 & ; & 238 & 334.69 & -0.4276 \\ \text {.word } & 52256 & ; & 239 & 336.09 & -0.4052 \\ \text {.word } & 52996 & ; & 240 & 337.50 & -0.3827 \\ \text {.word } & 53742 & ; & 241 & 338.91 & -0.3599 \\ \text {.word } & 54496 & ; & 242 & 340.31 & -0.3369 \\ \text {.word } & 55257 & ; & 243 & 341.72 & -0.3137 \\ \text {.word } & 56023 & ; & 244 & 343.13 & -0.2903 \\ \text {.word } & 56796 & ; & 245 & 344.53 & -0.2667 \\ \text {.word } & 57573 & ; & 246 & 345.94 & -0.2430 \\ \text {.word } & 58356 & ; & 247 & 347.34 & -0.2191 \\ \text {.word } & 59142 & ; & 248 & 348.75 & -0.1951 \\ \text {.word } & 59933 & ; & 249 & 350.16 & -0.1710 \\ \text {.word } & 60727 & ; & 250 & 351.56 & -0.1467 \\ \text {.word } & 61524 & ; & 251 & 352.97 & -0.1224 \\ \text {.word } & 62323 & ; & 252 & 354.38 & -0.0980 \\ \text {.word } & 63125 & ; & 253 & 355.78 & -0.0736 \\ \text {.word } & 63927 & ; & 254 & 357.19 & -0.0491 \\ \text {.word } & 64731 & ; & 255 & 358.59 & -0.0245 \\ \text {.word } & 65535 & ; & 256 & 360.00 & 0.0000\end{array}$

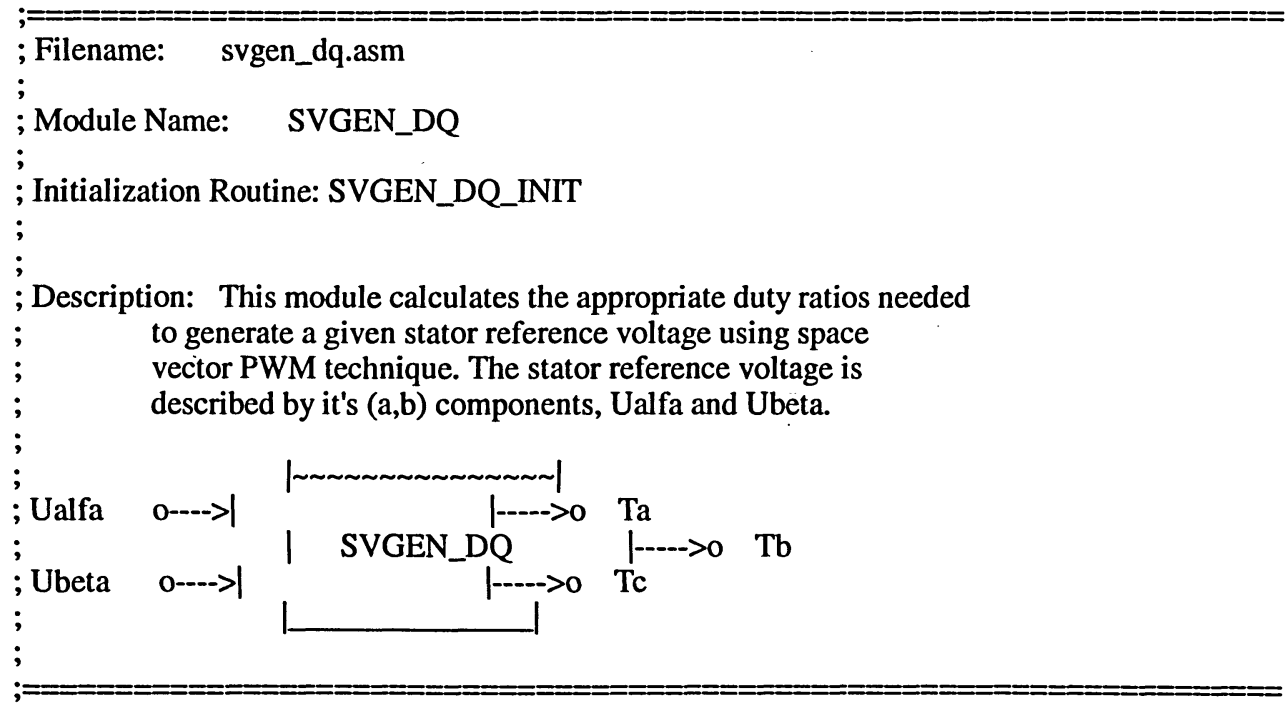

; History: 


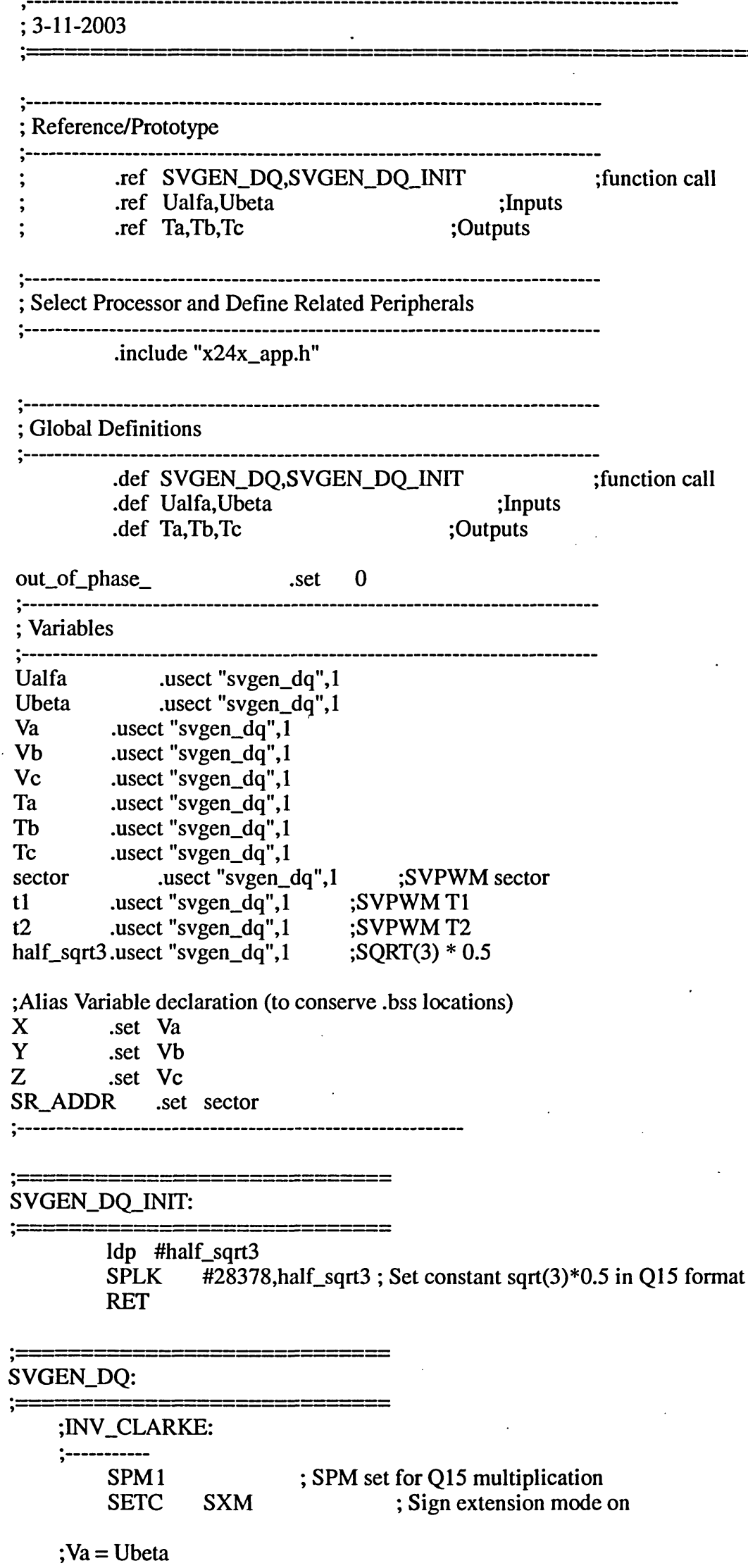




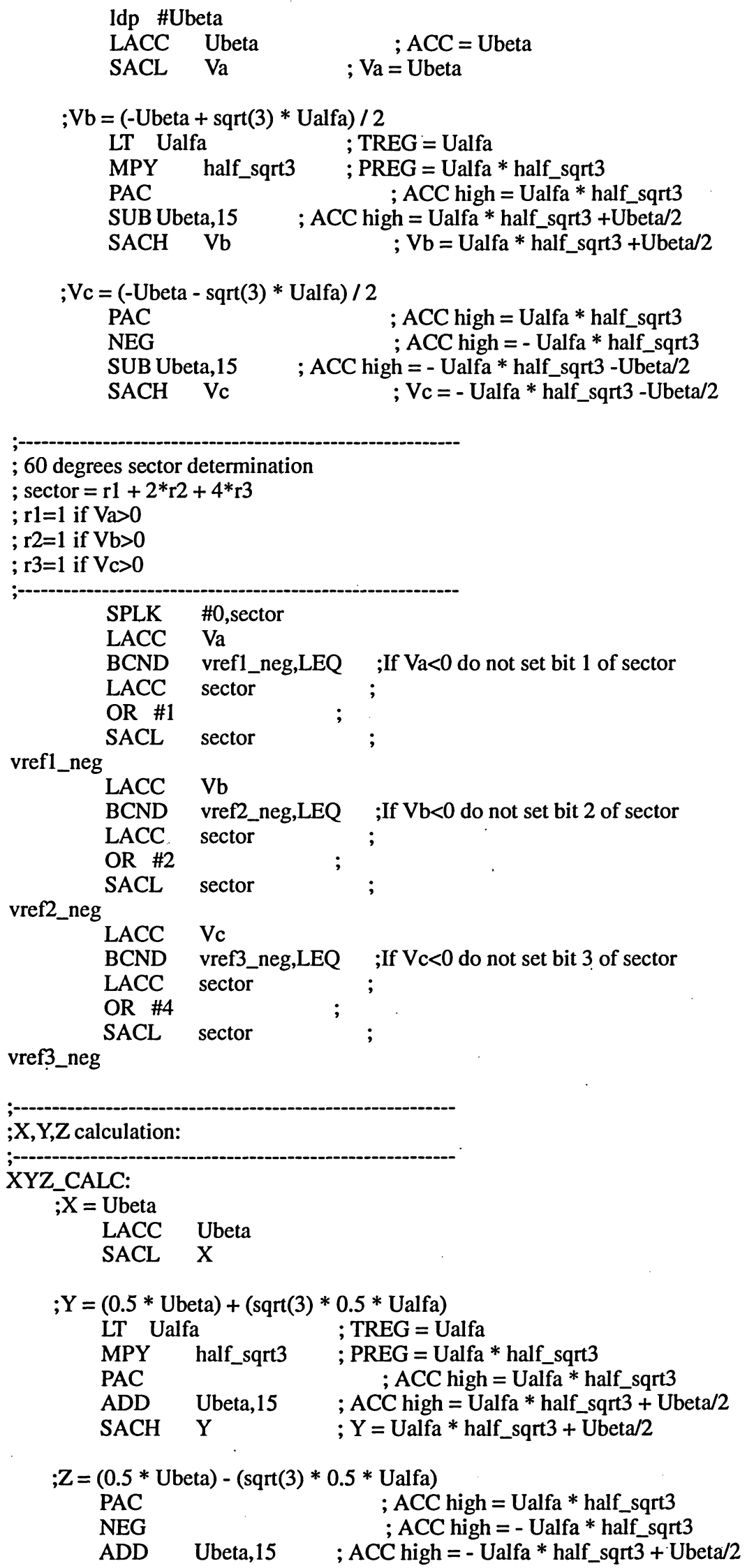


SACH Z $\quad ; Z=-$ Ualfa $*$ half_sqrt $3+$ Ubeta/2

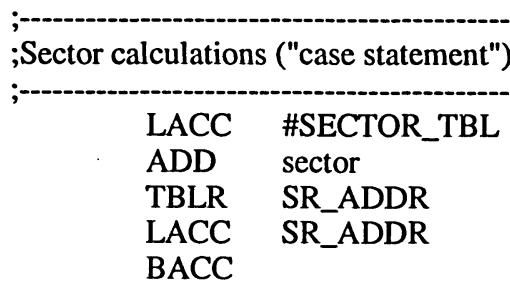

SECTOR_SR1:

;sector 1: $\mathrm{tl}=\mathrm{Z}$ and $\mathrm{t} 2=\mathrm{Y},(\mathrm{abc}-->\mathrm{Tb}, \mathrm{Ta}, \mathrm{Tc})$

lacc $\mathrm{Z}$

sacl $t 1$

lacc $\mathrm{Y}$

sacl t2

lacc \#7FFFh ;Load $1(\mathrm{Q} 15)$

sub t1

sub t2 ;taon $=(1-\mathrm{t} 1-\mathrm{t} 2) / 2$

sfr

sacl $\mathrm{Tb}$

add $\mathrm{tl}$; tbon=taon+t1

sacl Ta

add $\mathrm{t} 2 \quad$;tcon=tbon $+\mathrm{t} 2$

sacl Tc

B SV_END

SECTOR_SR2:

;sector 2: $\mathrm{t} 1=\mathrm{Y}$ and $\mathrm{t} 2=-\mathrm{X},(\mathrm{abc}-->\mathrm{Ta}, \mathrm{Tc}, \mathrm{Tb})$

lacc $\mathrm{Y}$

sacl $t 1$

lacc $\mathrm{X}$

neg

sacl $\mathrm{t} 2$

lacc \#7FFFh ;Load 1 (Q15)

sub $\mathrm{t} 1$

sub t2

sfr

sacl Ta

add $\mathrm{t} 1$

sacl Tc

add $\mathrm{t} 2 \quad$;tcon=tbon $+\mathrm{t} 2$

sacl $\mathrm{Tb}$

;taon $=(1-\mathrm{t} 1-\mathrm{t} 2) / 2$

;

;tbon=taon+t1

B SV_END

SECTOR_SR3:

;sector 3: $\mathrm{t} 1=-\mathrm{Z}$ and $\mathrm{t} 2=\mathrm{X},(\mathrm{abc}-->\mathrm{Ta}, \mathrm{Tb}, \mathrm{Tc})$

lacc $\mathrm{Z}$

neg

sacl $\mathrm{t} 1$

lacc $\mathrm{X}$

sacl t2

lacc \#7FFFh ;Load $1(\mathrm{Q} 15)$

sub t1

sub t2

sfr

;taon $=(1-\mathrm{t} 1-\mathrm{t} 2) / 2$ 


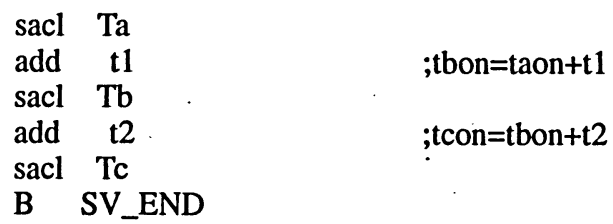

SECTOR_SR4:

;sector 4: $\mathrm{t} 1=-\mathrm{X}$ and $\mathrm{t} 2=\mathrm{Z},(\mathrm{abc}-->\mathrm{Tc}, \mathrm{Tb}, \mathrm{Ta})$ lacc $\mathrm{X}$

neg

sacl t1

lacc $\mathrm{Z}$

sacl t2

lacc \#7FFFh $\quad$;Load 1(Q15)

sub $\mathrm{t} 1$

sub t2 ;taon $=(1-\mathrm{t} 1-\mathrm{t} 2) / 2$

sfr

sacl Tc

add $\mathrm{t} 1 \quad$;tbon=taon+t1

sacl $\mathrm{Tb}$

add $\mathrm{t} 2$;tcon=tbon $+\mathrm{t} 2$

sacl Ta

B SV_END

SECTOR_SR5:

;sector 5: $\mathrm{t} 1=\mathrm{X}$ and $\mathrm{t} 2=-\mathrm{Y},(\mathrm{abc}-->\mathrm{Tb}, \mathrm{Tc}, \mathrm{Ta})$

lacc $X$

sacl $\mathrm{t} 1$

lacc $\mathrm{Y}$

neg

sacl $\mathrm{t} 2$

lacc \#7FFFh ;Load 1 (Q15)

sub $\mathrm{t} 1$

sub t2

sfr

sacl $\mathrm{Tb}$

add $\mathrm{t} 1$

sacl Tc

add $\mathrm{t} 2$

sacl Ta

B. SV_END

SECTOR_SR6:

;sector 6: $\mathrm{t} 1=-\mathrm{Y}$ and $\mathrm{t} 2=-\mathrm{Z},(\mathrm{abc}-->\mathrm{Tb}, \mathrm{Tc}, \mathrm{Ta})$

lacc $\mathrm{Y}$

neg

sacl $\mathrm{t} 1$

lacc $\mathrm{Z}$

neg

sacl t2

lacc \#7FFFh ;Load 1(Q15)

sub $t 1$

sub t2

sfr

sacl Tc

add $\mathrm{t} 1$

;taon $=(1-\mathrm{t} 1-\mathrm{t} 2) / 2$

;tbon=taon+t1

;tcon=tbon+t2

Z

;Load 1 (Q15)

;taon $=(1-\mathrm{t} 1-\mathrm{t} 2) / 2$

;tbon=taon+t1 
sacl $\mathrm{Ta}$

add $\mathrm{t} 2 \quad$;tcon=tbon+t2

sacl $\mathrm{Tb}$

SV_END:

;Multiply Ta by $2 \&$ offset by $1 / 2$

LACC Ta

SUB \#3FFFh

SACL Ta,1 ;mpy by 2

;Multiply Tb by $2 \&$ offset by $1 / 2$

LACC Tb

SUB \#3FFFh

SACL Tb,1. ;mpy by 2

;Multiply Tc by $2 \&$ offset by $1 / 2$

LACC Tc

SUB \#3FFFh

SACL Tc, 1 ;mpy by 2

.if (out_of_phase_)

LACC Ta

NEG

SACL Ta

LACC $\cdot \mathrm{Tb}$

NEG

SACL Tb

LACC Tc

NEG

.endif

SACL Tc

DUMMY $\underset{\text { RET }}{\text { SPM0 }}$; SPM reset

;SVPWM Sector routine jump table - used with BACC inst.

\begin{tabular}{lll} 
SECTOR_TBL: & \\
SR00 & \multicolumn{3}{c}{.word } & DUMMY \\
SR0 & .word & SECTOR_SR1 \\
SR1 & .word & SECTOR_SR2 \\
SR2 & .word & SECTOR_SR3 \\
SR3 & .word & SECTOR_SR4 \\
SR4 & .word & SECTOR_SR5 \\
SR5 & .word & SECTOR_SR6
\end{tabular}




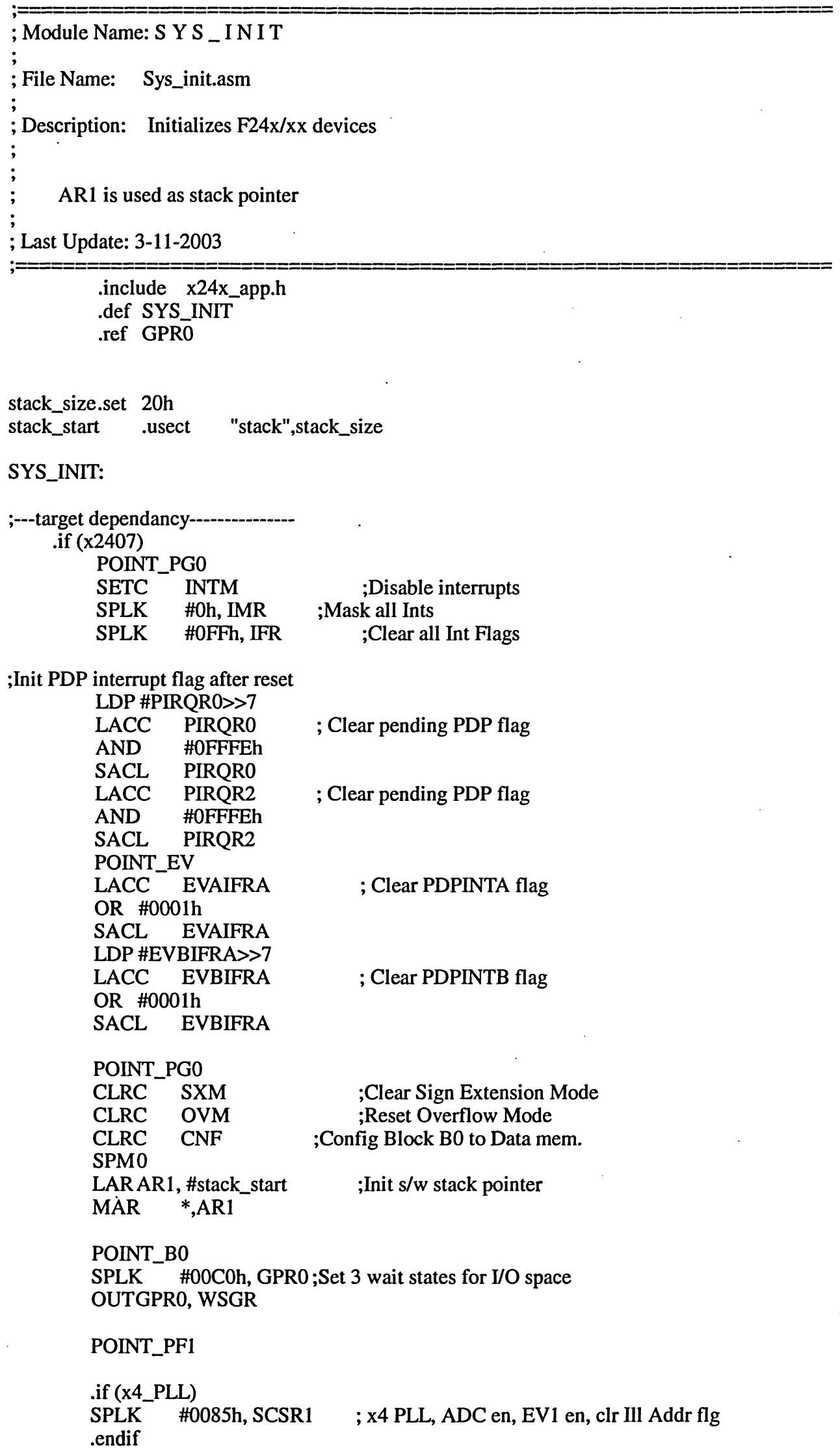


if (x2_PLL)

SPLK \#0285h, SCSR1 ; $\quad$ 2 PLL, ADC en, EV1 en, clr Ill Addr flg .endif

;Comment out if WD is to be active

SPLK \#006Fh, WD_CNTL ;Disable WD if VCCP=5V

KICK_DOG

.endif

RET

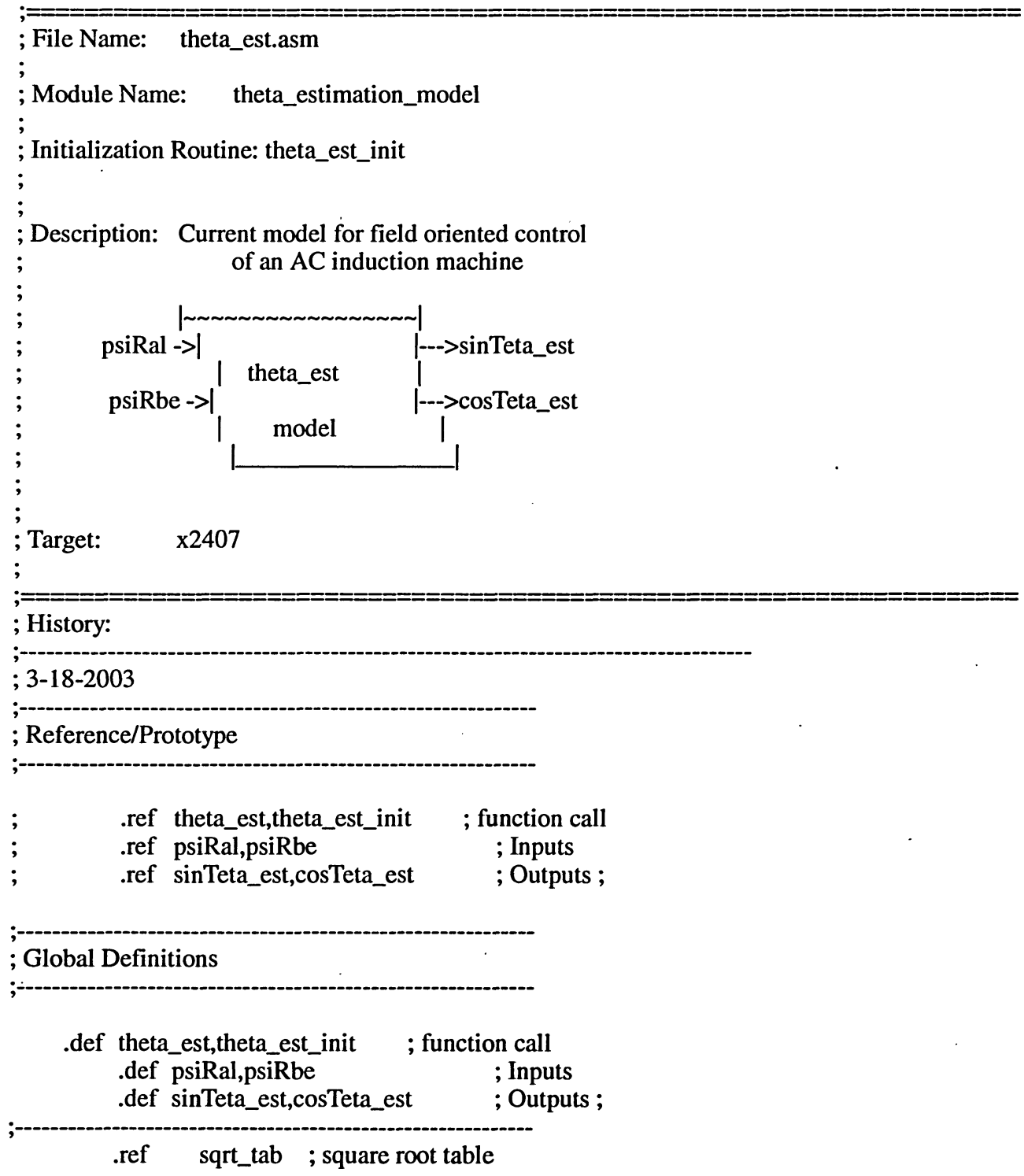




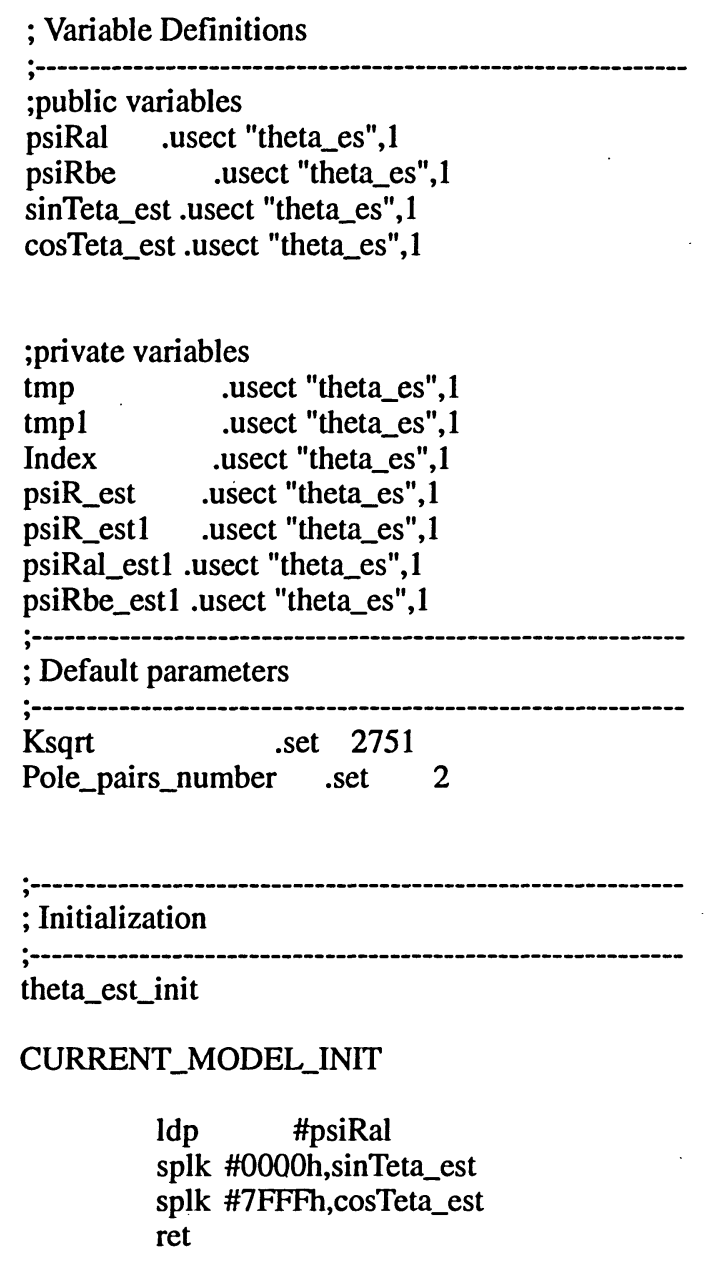

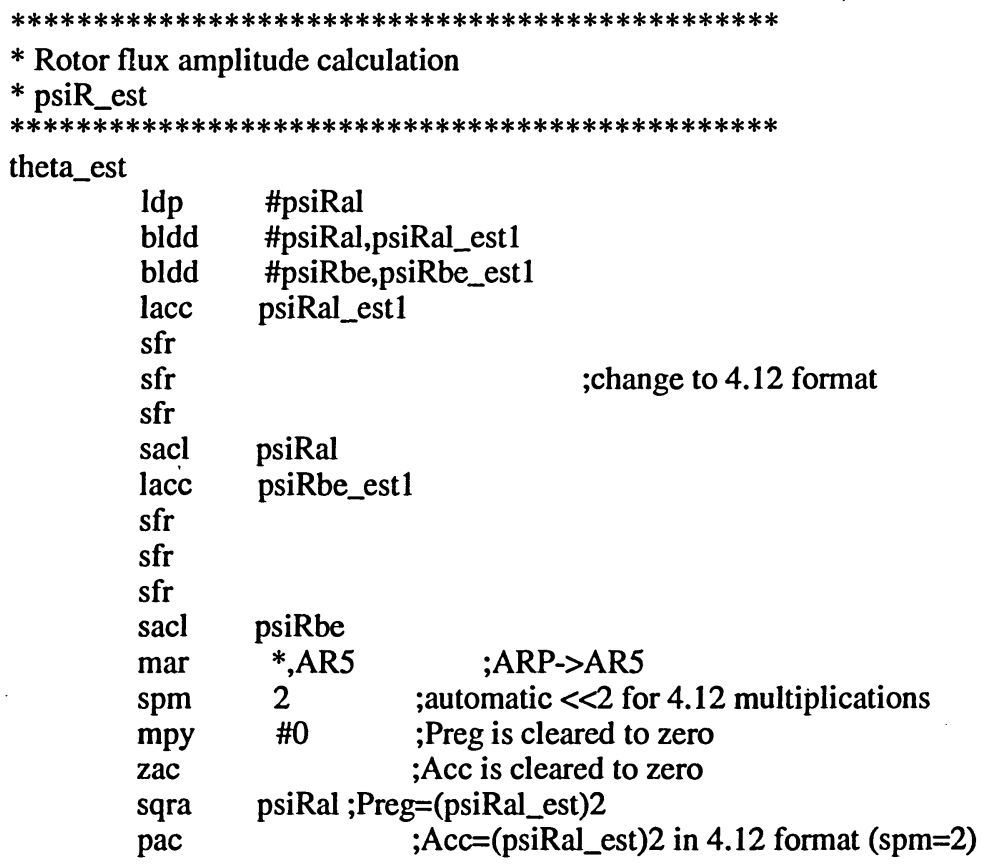


* sfr sacl lacc bcnd lacc neg modzerol sinpos ;three right shifts $\rightarrow$ division result in 4.12 format sinTeta_est ;store 1.15 res (=psiRbe_est $\left.1 / p s i R \_e s t 1\right)$ in sinTeta_est psiRbe_est1 sinpos,GT sinTeta_est

;If psiRbe_est $1<0$ then sinTeta_est $<0$

$* * * * * * * * * * * * * * * * * * * * * * * * * * * * * * *$

* END divisions

$* * * * * * * * * * * * * * * * * * * * * * * * * * * * * * *$

ret

$20-123 \cdot 52$ 